\title{
ANALYSIS OF NECK MUSCLE ACTIVITY AND COMPARISON OF HEAD AND BODY MOTION DURING ROTATIONAL MOVEMENT IN A MOTION SIMULATOR
}

\author{
by \\ Shahini Sirikantharajah \\ B.Eng., Ryerson University, 2013 \\ A thesis \\ presented to Ryerson University \\ in partial fulfillment of the \\ requirements for the degree of \\ Master of Applied Science \\ in the Program of \\ Electrical and Computer Engineering
}

Toronto, Ontario, Canada, 2015

(C)Shahini Sirikantharajah, 2015 


\section{AUTHOR'S DECLARATION FOR ELECTRONIC SUBMISSION OF A THESIS}

I hereby declare that I am the sole author of this thesis. This is a true copy of the thesis, including any required final revisions, as accepted by my examiners.

I authorize Ryerson University to lend this thesis to other institutions or individuals for the purpose of scholarly research.

I further authorize Ryerson University to reproduce this thesis by photocopying or by other means, in total or in part, at the request of other institutions or individuals for the purpose of scholarly research.

I understand that my thesis may be made electronically available to the public. 


\begin{abstract}
Analysis of Neck Muscle Activity and Comparison of Head and Body Motion During Rotational Movement in a Motion Simulator, Master of Applied Science, 2015

Shahini Sirikantharajah

Department of Electrical and Computer Engineering, Ryerson University.
\end{abstract}

Most of the research relating to neck injuries performed to date has been tested in environments with linear motions. Disabled individuals tend to experience jerky neck rotations during falls, bed transfers, and while travelling in wheelchairs. This thesis, using various signal processing techniques, studied how healthy neck muscles, the head and body react to jerky rotational motion. Electromyogram (EMG) and motion data were gathered from 20 subjects as they were rotated 45 degrees in the forward and backward pitch plane, with and without visual input, in a motion simulator.

Results showed that neck muscle behaviour was affected by the direction of motion and visual input. Maximum effective muscle power of $10.54 \%$ was reached, relative to maximum voluntary contractions (MVC). The factors found to influence neck muscle responses, such as head weight and visual input should be taken into consideration when designing headrests, neck braces and planning any rehabilitation programs. 


\section{Acknowledgment}

I would like to start off by expressing my sincere appreciation and gratitude to my supervisor Dr.

Kristiina M. Valter McConville for her guidance and encouragement throughout this project. Her feedback and tips gave me confidence and motivation, every step of the way. Thanks to Yu Wai Chau for helping out with data acquisition. I would also like to thank Chanda Malik and Himel Khandker for their help recruiting volunteers and test subjects for this study. Finally, thanks to my defense committee, Dr. Kyan, Dr. Raahemifar, and Dr. Guan, for their valuable input and feedback. 


\section{Dedication}

This thesis is dedicated to my parents and my brother. Without their love, support, and guidance, I would not have made it this far. 


\section{Contents}

1 Introduction 1

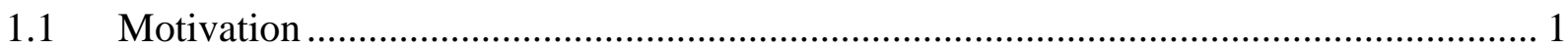

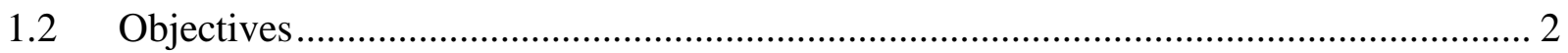

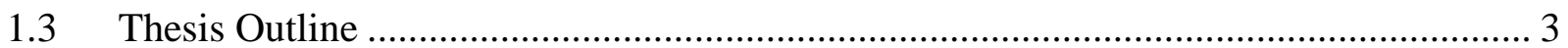

2 Background Information $\quad 4$

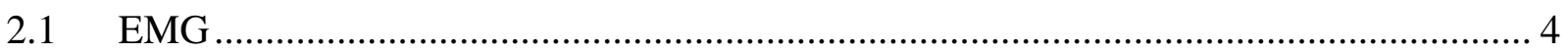

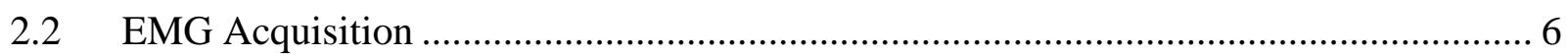

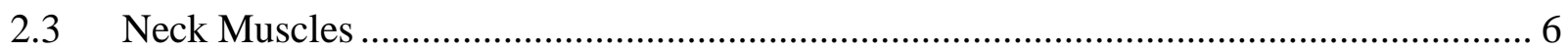

2.3.1 Injuries and Disorders Affecting the Neck ................................................. 7

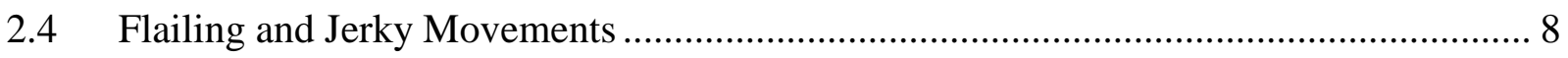

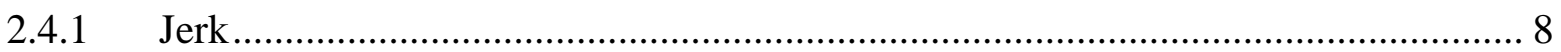

2.4.2 Computation of Jerk Systems ........................................................................ 9

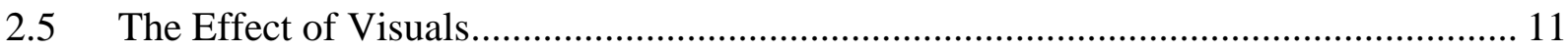

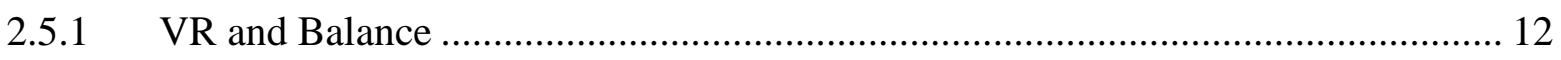

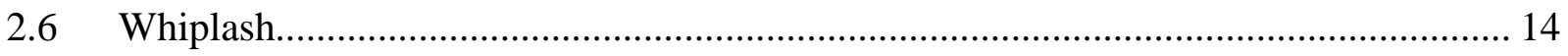

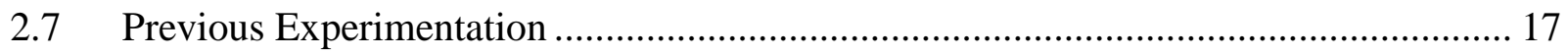

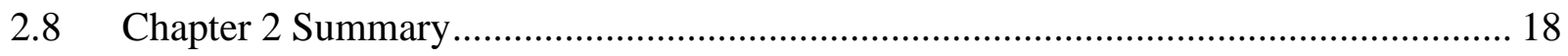

3 Experimental Setup and Methods $\quad 19$

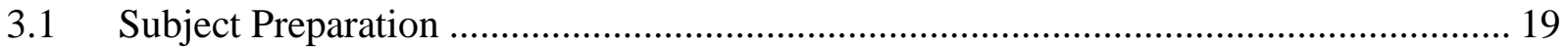

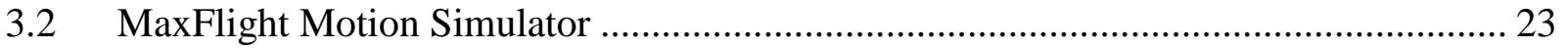

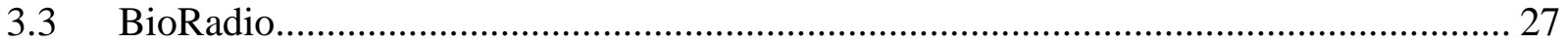

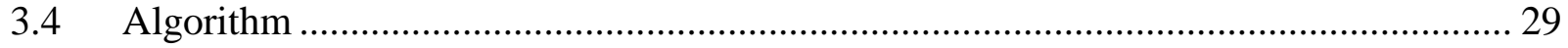

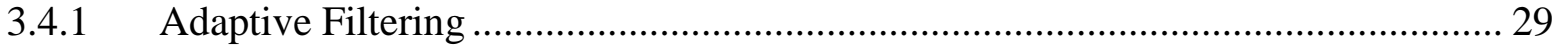

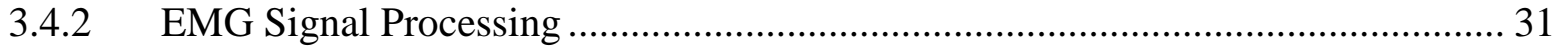

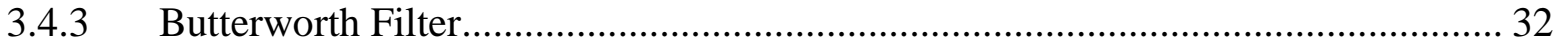

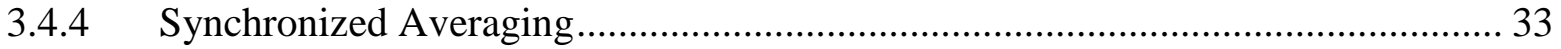




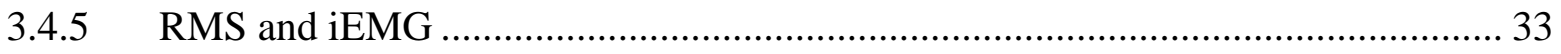

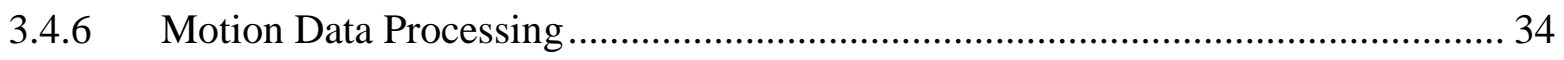

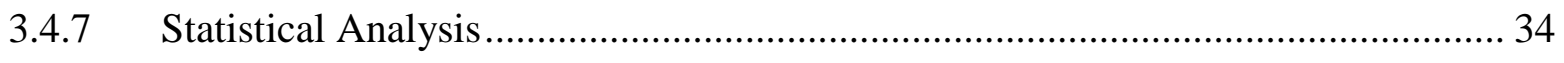

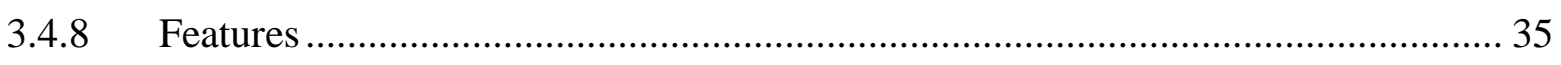

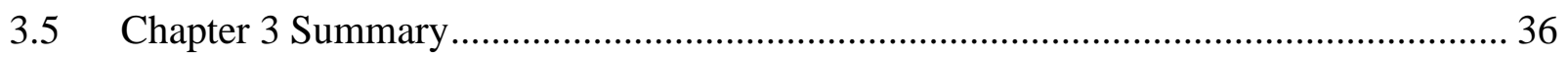

4 Results and Discussion $\quad 37$

4.1 Graphical Results of Neck Muscle Activity …….......................................................... 37

4.1.1 Adaptive Filtering Results ................................................................................. 37

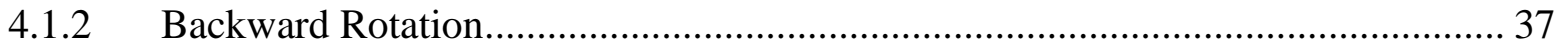

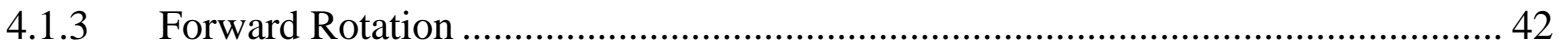

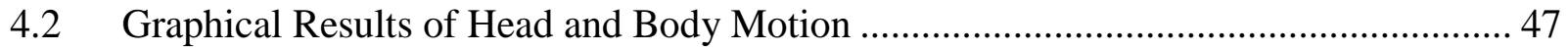

4.2.1 Backward Rotation......................................................................................... 47

4.2.2 Forward Rotation ..................................................................................... 58

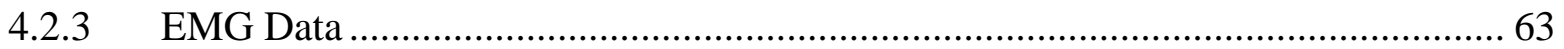

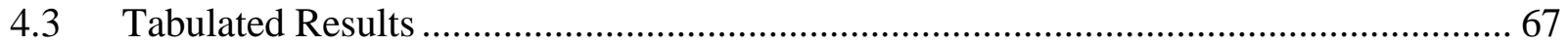

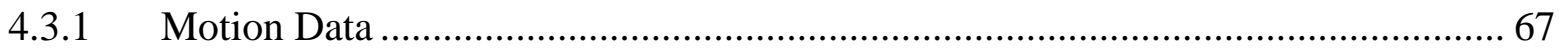

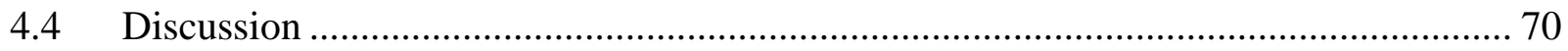

4.4.1 Comparison to Previous Experimentation ....................................................... 72

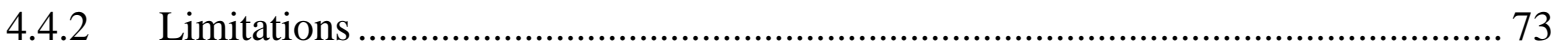

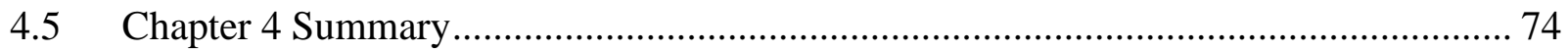

5 Conclusions and Future Work $\quad 75$

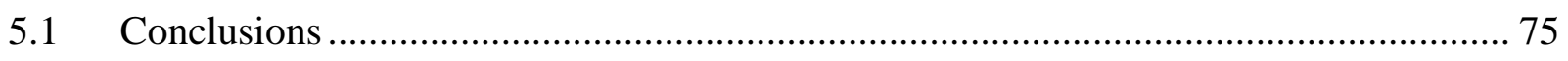

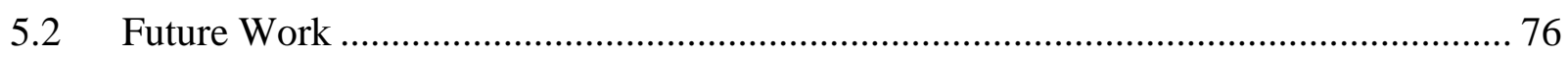

$\begin{array}{ll}\text { A Experimental Results } & \mathbf{7 7}\end{array}$

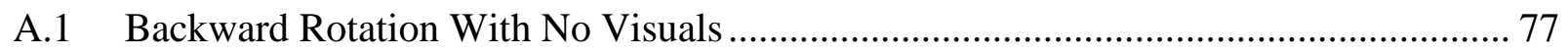

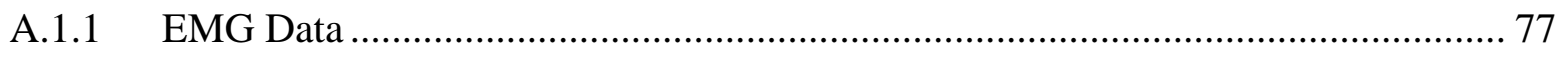

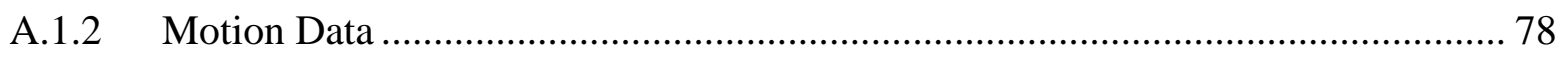

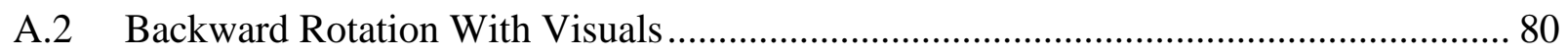

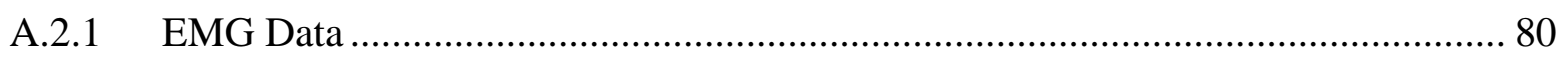

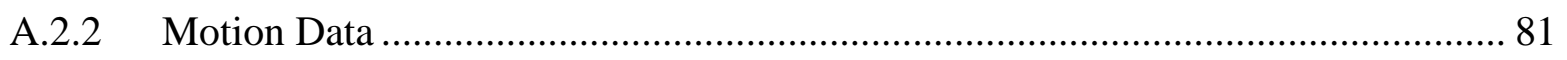

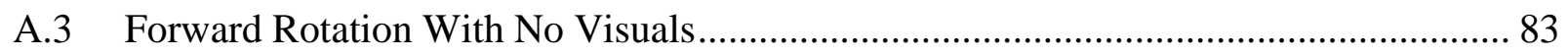

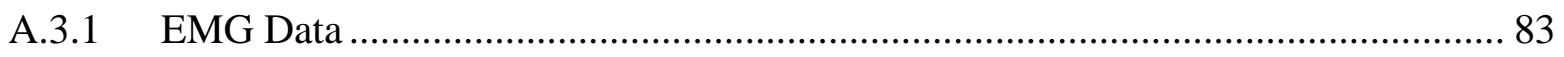


A.3.2 Motion Data ..................................................................................... 84

A.4 Forward Rotation With Visuals...................................................................... 86

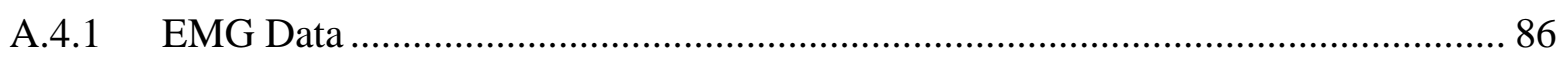

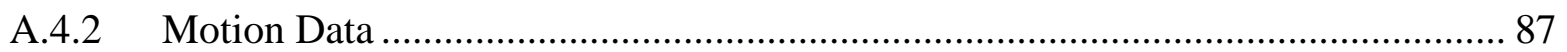

$\begin{array}{lr}\text { B Publications } & 89\end{array}$

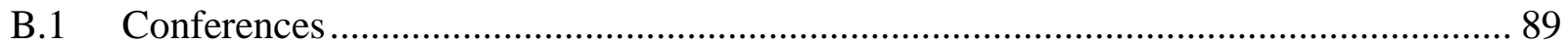

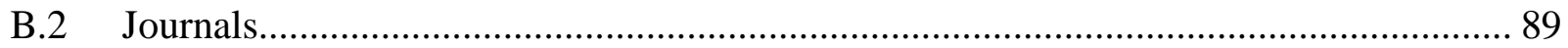

$\begin{array}{ll}\text { Bibliography } & 90\end{array}$

$\begin{array}{ll}\text { Glossary } & 98\end{array}$ 


\section{List of Tables}

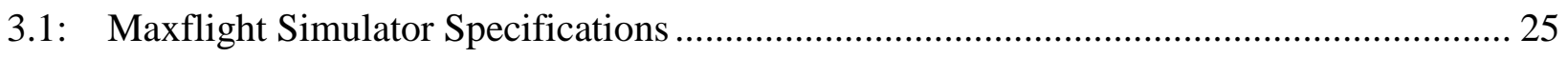

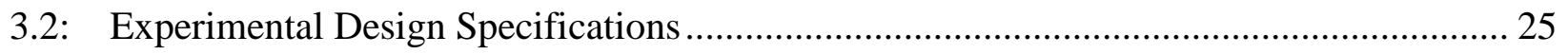

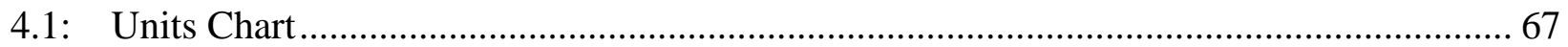

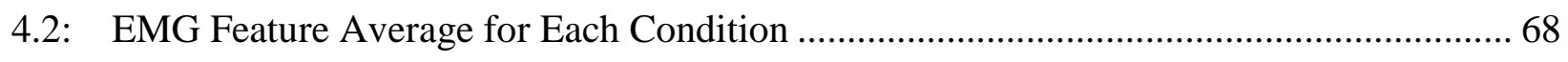

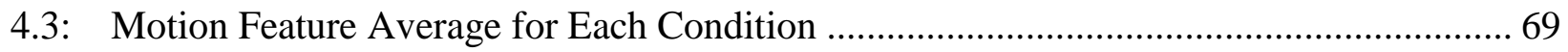

4.4: Head Weight to Motion Comparison........................................................................ 70

A.1: $\quad$ EMG Feature Data for Backward Rotation with no Visuals ........................................ 77

A.2: Motion Feature Data for Backward Rotation with no Visuals ..................................... 84

A.3: EMG Feature Data for Backward Rotation with Visuals ............................................ 85

A.4: Motion Feature Data for Backward Rotation with Visuals ......................................... 86

A.5: EMG Feature Data for Forward Rotation with no Visuals ......................................... 87

A.6: Motion Feature Data for Forward Rotation with no Visuals ........................................ 88

A.7: EMG Feature Data for Forward Rotation with Visuals ............................................ 89

A.8: Motion Feature Data for Forward Rotation with Visuals .......................................... 90 


\section{List of Figures}

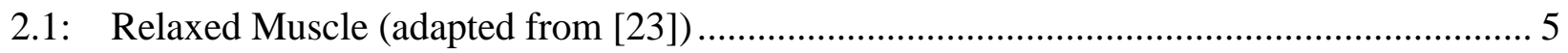

2.2: Contracted Muscle (adapted from [23]) ........................................................................ 5

2.3: Neck Muscles (taken from [23]) ............................................................................... 7

2.4: Angular Motion Profile of Simulator................................................................................ 10

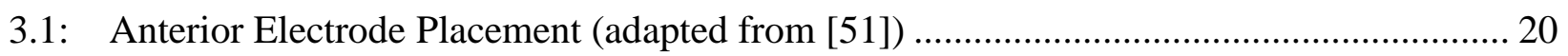

3.2: Posterior Electrode Placement (adapted from [51]) ……………………………............. 21

3.3: Accelerometer Placement (taken from [23]) ………....................................................... 21

3.4: Visual Display of Tunnel Moving Forward ..................................................................... 23

3.5: Three Dimensions of Motion (taken from [52]) ……...................................................... 24

3.6: Subject Rotated in the Backward Pitch Plane (left) and Forward Pitch Plane (right) ......... 24

3.7: Motion Simulator at 45 degrees forward (left) and 45 degrees backward (right) ............... 25

3.8: Motion Simulator at Neutral Position .................................................................................... 26

3.9: Motion Simulator with Canopy Open.......................................................................... 26

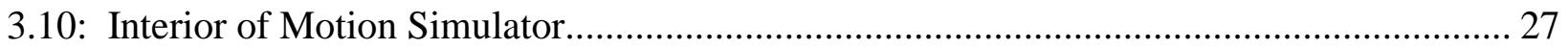

3.11: BioRadio Axes (adapted from [57]) …………….................................................... 28

3.12: Adaptive Filter Block Diagram (adapted from [24]) …................................................... 30

3.13: EMG Signal Processing Block Diagram .......................................................................... 31

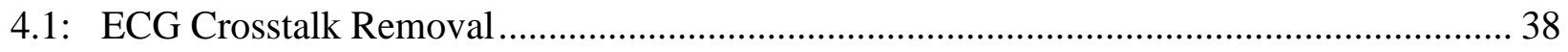

4.2: $\quad$ EMG for Backward Rotation with no Visuals (red = envelope; blue = EMG) .................... 39

4.3: $\quad$ EMG for Backward Rotation with Visuals (red = envelope; blue = EMG) ........................ 41

4.4: iEMG for Backward Rotation with no Visuals............................................................... 43

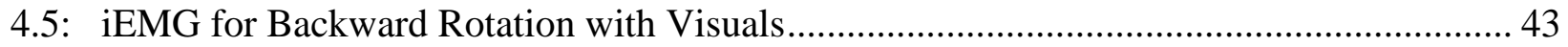

4.6: $\mathrm{EMG}$ for Forward Rotation with no Visuals (red = envelope; blue = EMG) ...................... 44 
4.7: EMG for Forward Rotation with Visuals (red = envelope; blue = EMG) ...................... 45

4.8: iEMG for Forward Rotation with no Visuals .............................................................. 48

4.9: iEMG for Forward Rotation with Visuals ............................................................... 48

4.10: All EMG for Backward Rotation with no Visuals...................................................... 49

4.11: All EMG for Backward Rotation with Visuals........................................................... 49

4.12: All EMG for Forward Rotation with no Visuals ....................................................... 50

4.13: All EMG for Forward Rotation with Visuals ......................................................... 50

4.14: Motion Profile of Neck for Backward Rotation with no Visuals (red = pitch; blue = yaw;

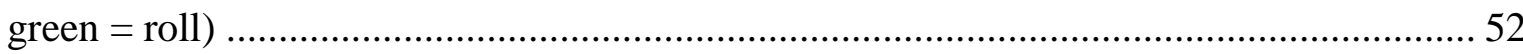

4.15: Motion Profile of Body for Backward Rotation with no Visuals (red = pitch; blue = yaw;

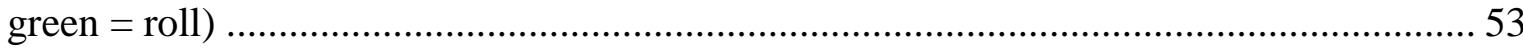

4.16: Comparison of EMG, Neck and Body Angular Velocity for Backward Rotation with no

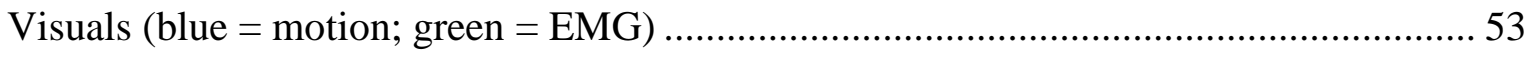

4.17: All Differential Motion Graphs for Backward Rotation with no Visuals........................ 54

4.18: Average Differential Motion Graphs for Backward Rotation with no Visuals ................. 55

4.19: Motion Profile of Neck for Backward Rotation with Visuals (red = pitch; blue = yaw; green

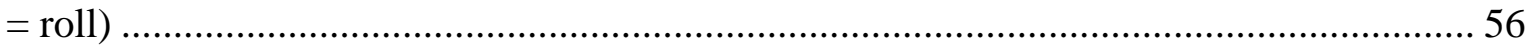

4.20: Motion Profile of Body for Backward Rotation with Visuals (red = pitch; blue = yaw;

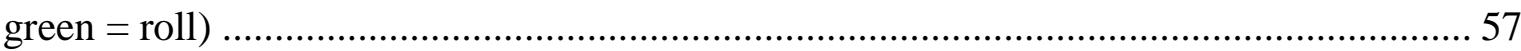

4.21: Comparison of EMG, Neck and Body Angular Velocity for Backward Rotation with

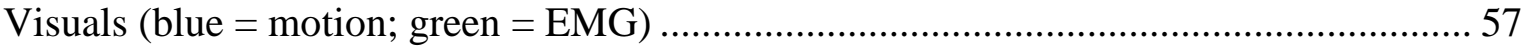

4.22: All Differential Motion Graphs for Backward Rotation with Visuals............................ 58

4.23: Average Differential Motion Graphs for Backward Rotation with Visuals ...................... 59

4.24: Motion Profile of Neck for Forward Rotation with no Visuals (red = pitch; blue = yaw;

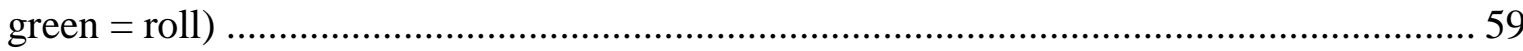

4.25: Motion Profile of Body for Forward Rotation with no Visuals (red = pitch; blue = yaw; green $=$ roll $)$

4.26: Comparison of EMG, Neck and Body Angular Velocity for Forward Rotation with no

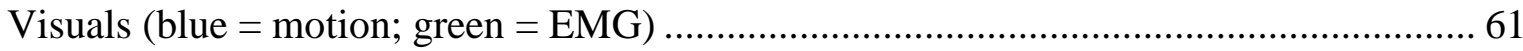

4.27: All Differential Motion Graphs for Forward Rotation with no Visuals .......................... 62

4.28: Average Differential Motion Graphs for Forward Rotation with no Visuals................... 62 
4.29: Motion Profile of Neck for Forward Rotation with Visuals (red = pitch; blue = yaw; green $=$ roll)

4.30: Motion Profile of Body for Forward Rotation with Visuals (red = pitch; blue = yaw; green $=$ roll) 64

4.31: Comparison of EMG, Neck and Body Angular Velocity for Forward Rotation with Visuals (blue $=$ motion; green $=\mathrm{EMG})$ 65

4.32: All Differential Motion Graphs for Forward Rotation with Visuals 65

4.33: Average Differential Motion Graphs for Forward Rotation with Visuals 66

A.1: Acceleration and Jerk Profiles of Neck for Backward Rotation with no Visuals. 79

A.2: Acceleration and Jerk Profiles of Body for Backward Rotation with no Visuals 79

A.3: Acceleration and Jerk Profiles of Neck for Backward Rotation with Visuals 82

A.4: Acceleration and Jerk Profiles of Body for Backward Rotation with Visuals 82

A.5: Acceleration and Jerk Profiles of Neck for Forward Rotation with no Visuals 85

A.6: Acceleration and Jerk Profiles of Body for Forward Rotation with no Visuals 85

A.7: Acceleration and Jerk Profiles of Neck for Forward Rotation with Visuals 88

A.8: Acceleration and Jerk Profiles of Body for Forward Rotation with Visuals 88 


\section{Chapter 1}

\section{Introduction}

\subsection{Motivation}

OME of the most serious emergency room cases are related to falls, injuries and other accidents. According to Statistics Canada, falls are the primary cause of injuries [1]. A study performed in 2010 revealed that $63 \%$ of seniors have been injured by falls, out of which $55 \%$ occurred while walking [1]. Disabled individuals affected by conditions such as spinal cord injury (SCI) and multiple sclerosis are highly prone to movement disorders and balance impairments. Mobility assistive technologies (MAT) such as wheelchairs help disabled individuals safely travel from one point to another. One of the common areas of research related to both seating and wheelchair use is posture and balance. Continued wheelchair use has been found to cause injuries, such as joint pain, tendonitis, and carpal tunnel syndrome [2]. Many individuals may already have weaker muscles due to spinal cord injuries (SCI) and muscular dystrophic conditions [3-7].

The neck plays a very important role in maintaining head balance and good posture. Simple daily activities such as moving from bed to wheelchair can induce strain on the neck, especially if such activities result in falls. Although not to a greater degree, travelling over uneven surfaces and ramps can also cause whiplash injuries, due to rotational jerky motions. When individuals are tipped backward or forward, the neck and body undergo different motions as the individual shifts his/her centre of mass [2]. Many studies have been performed to examine how upper limb muscles react during whiplash [8-13]. These studies tested head and neck movements during various activities, as well as during jerky motions caused by linear experimental sleds. Most of these studies had applied their research towards car collisions. 
Other studies have analyzed upper body movement of disabled patients as they are transfer from bed to wheelchair [14-16]. Yet, not much research has focused on how neck muscles react to rotational motions. The neck muscles are essential during propulsion, especially when trying to retain an upright position and a good view of the surroundings [10]. In a study conducted by Vibert N. et al., the EMG of neck muscles were assessed during varying jerk and accelerations on a linear sled [17]. Many subjects showed head movements that were in the opposite direction of the sled movement (common to whiplash injuries). Hence, this thesis will further investigate the activity of neck muscles and compare neck movement in relation to the body, during forward and backward rotational movements. Another factor to consider would be the effect of additional sensory inputs towards muscle behaviour, since head motion can also depends on one's focus ahead. An disconnection between the visual and vestibular information perceived by an individual can affect his/her awareness of orientation and motion [18]. Hence, this thesis will also study neck motion with and without the presence of virtual reality (VR).

As wheelchair users travel over rough surfaces and ramps, an oscillation in motion can be seen as the body sways back and forth to avoid tipping over. The amount of body sway has been associated to abdominal muscle strength [2]. Similarly, the strength of neck muscles may also be associated with the amount of head and neck propulsion. Wheelchair users affected by SCI or muscular dystrophy, tend to have trouble with head and neck stabilization and posture. Since very few studies have analyzed neck motion and neck muscle behaviour in rotational motions, this thesis aims to start by analyzing healthy muscles behaviours under such motions. Additionally, this thesis aims to compare head motion to body sway under rotational motions. According to the sensory conflict theory, motion sickness often occurs when there is conflict between visual and vestibular inputs [18-20]. Motion sickness may also result in difficulty of neck and body stabilization, as well as loss of control.

\subsection{Objectives}

The primary objective of this study was to investigate Neck EMG during rotational motions, with and without the presence of VR. The secondary objective was to compare the head and body motions simultaneously, under the same conditions. The study also considered 
factors such as head mass and neck size, in order to determine whether these factors influenced the results.

Neck muscle responses are expected to be greatest near the beginning and end of motion, as these periods have higher levels of jerk. It is also hypothesized that the presence of VR will have a noticeable effect on both head motion and neck muscle activity, since the visual and vestibular systems work together to help provide consciousness of body position and motion.

\subsection{Thesis Outline}

The thesis outline is as follows:

- Chapter 2: This chapter discusses background information relating to EMG and how they are affected during jerk. It also provides information about neck muscles and types of neck conditions and disorders. Finally, the chapter provides a literature review of research performed to date in the area of whiplash injuries, VR and balance.

- Chapter 3: This chapter will describe the devices used in the study and the procedures followed during testing. The chapter will also go into the details of signal processing techniques and methodologies used to analyze the data.

- Chapter 4: This chapter presents both graphical and tabulated EMG and motion results for the four conditions that were tested: (1) backward with no visuals, (2) backward with visuals, (3) forward with no visuals, and (4) forward with visuals. Results are discussed, and compared amongst each other and previous research work. The chapter ends of by discussing limitations in the study.

- Chapter 5: The final chapter will summarize the thesis, identify how it can be built on, and list potential applications. 


\section{Chapter 2}

\section{Background Information}

\subsection{EMG}

The human body consists of three types of muscles: skeletal, cardiac and visceral [21, 22]. 1 Muscles are made up of fibers, which contain thin and thick filaments [23]. These filaments slide past each other during contraction. The sliding activity of these filaments are caused by the passing of ions through the muscle membrane [23]. Skeletal muscles are controlled voluntarily by the somatic nervous system. The movement of specific body parts result in the contraction of associated muscles. The degree of contraction and the amount of force exhibited depends on the signal sent via the somatic nervous system. An EMG records the electrical activity of muscles and can be used to study the difference in activity during rest and contractions. Figures 2.1 and 2.2 show the difference between a relaxed and contracted muscle. The protein bands that make up a muscle begin to overlap each other, as muscle contractions occur.

There are different types of muscle contractions that result in different signals patterns. A simple twitch contraction is caused by a single nerve impulse, while numerous nerves impulses causes a summation [23]. A summation of nerve impulses produces a longer lasting contraction. Very small contractions are referred to as isometric. They do not exert sufficient force to cause movement of a body part; however isometric contractions do play a role in muscle tension [23]. 


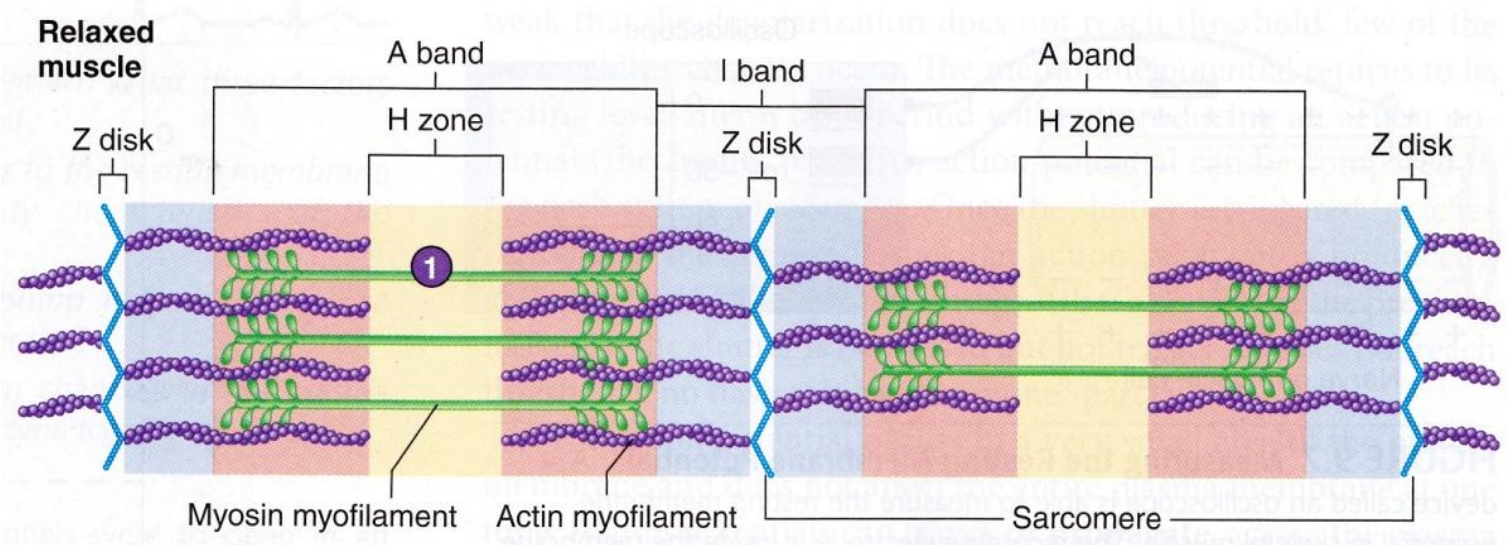

Figure 2.1: Relaxed Muscle (adapted from [23])

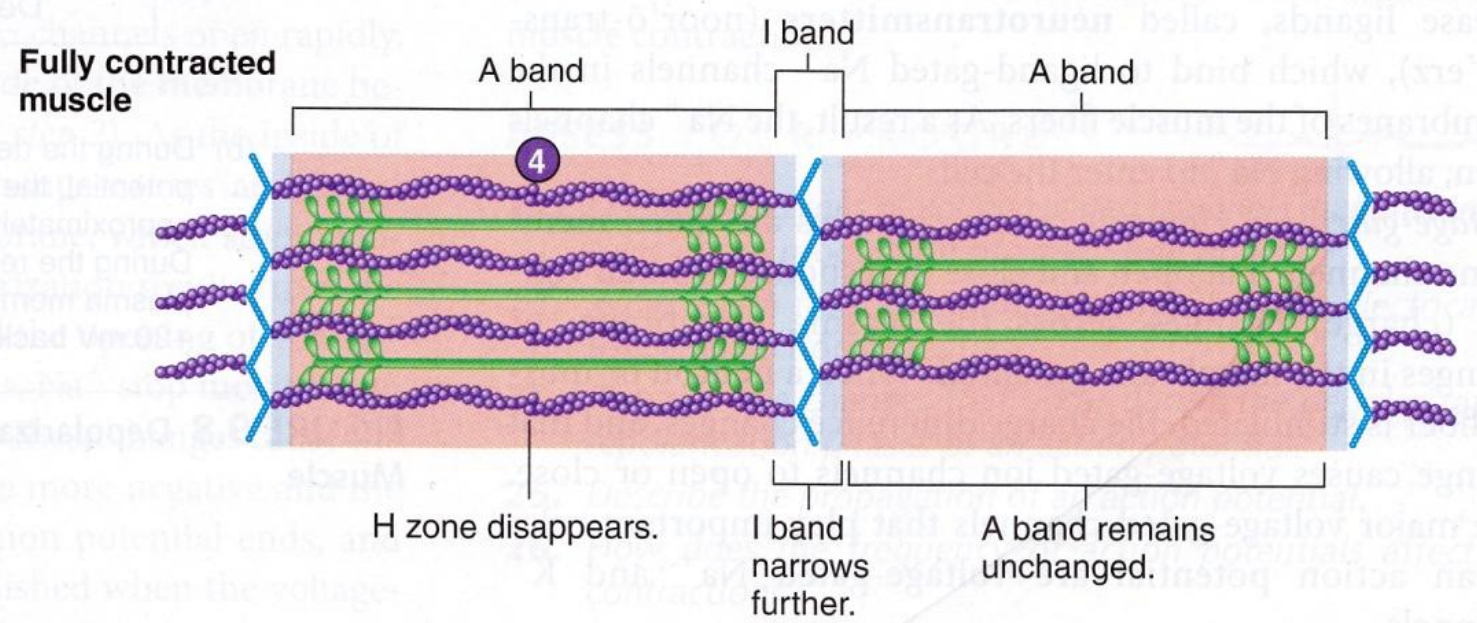

Figure 2.2: Contracted Muscle (adapted from [23]) 


\subsection{EMG Acquisition}

The EMG can be measured invasively using needle electrodes or non-invasively using surface electrodes. The magnitude of an EMG signal can range from $\mu \mathrm{V}$ to $\mathrm{mV}$ depending on the measuring system, electrode placement and contraction type [24]. Some of the important factors that need to be considered when acquiring EMG data include skin preparation, electrode impedance, transducer noise, and the influence of surrounding muscle signals. $\mathrm{Ag}-\mathrm{AgCl}$ electrodes use silver metal and silver chloride to decrease any impedance effects [24].

These specific type of electrodes are disposable and are widely used for biomedical and clinical signal acquisition. Many of the EMG acquisition devices come with built in amplifiers that work to amplify the difference between electrode potentials. This allows the noise created by electrode-to-skin interaction amongst the electrodes to be suppressed. Another important factor is to make sure the skin electrode impedance remains constant throughout the entire recording. Impedance has also been found to be reduced by proper skin preparation. The interference of surrounding muscles signals can be minimized by reducing the inter-electrode distance, as well as the conductive area [24]. This results in a smaller EMG measuring distance, which is in turn affected by a smaller radius of surrounding muscles.

Since the magnitude and amplitudes of EMG signals can vary between different acquisition systems and subjects, normalization of the data is essential in order to analyze and compare data. There are various methods that can be used for normalization. One example is to relate the signal to the degree of contraction or the force applied [25]. When positioning the electrodes they can be placed perpendicularly or with respect to the long axis of the specific muscle. Additionally, signals between subjects can be directly compared once they have been normalized using the subjects' maximum voluntary contractions (MVC).

\subsection{Neck Muscles}

Neck muscles are a very delicate and vital part of the body, as they are responsible for head and neck movement. This gross motor movement is essential for head posture, which aids vision, and ultimately the safe navigation of humans. Some of the major muscles involved with 
neck flexion and rotation are sternocleidomastoid (SCM), trapezius (TRAP), scalene (SCAL), splenius capitis, and semispinalis capitis [23]. These muscles, seen in Figure 2.3, work in pairs from either side of the neck. When the SCM muscle is contracted on one side only, the head and neck is either laterally flexed to the same side of the contracted muscle or rotated to the opposing side of the contracted muscle. When the muscles on both sides of the neck are contracted together, the head and neck are flexed and extended. The splenius capitis and semispinalis capitis are deeper muscles that are overlapped by TRAP muscles [23]. These muscles also help with neck extension and rotation. The TRAP muscle has the function of extending and laterally flexing the neck, while the SCAL rotates and laterally flexes the neck.

\subsubsection{Injuries and Disorders Affecting the Neck}

Many individuals become confined to wheelchairs due to SCI or other conditions, such as muscular dystrophy, multiple sclerosis, etc. Many wheelchair users have weak muscles, as a result of SCI or atrophy, which impacts their neck muscle responses [2]. Patients affected by SCI tend to experience head and neck pressure. Neck injuries can also affect breathing.

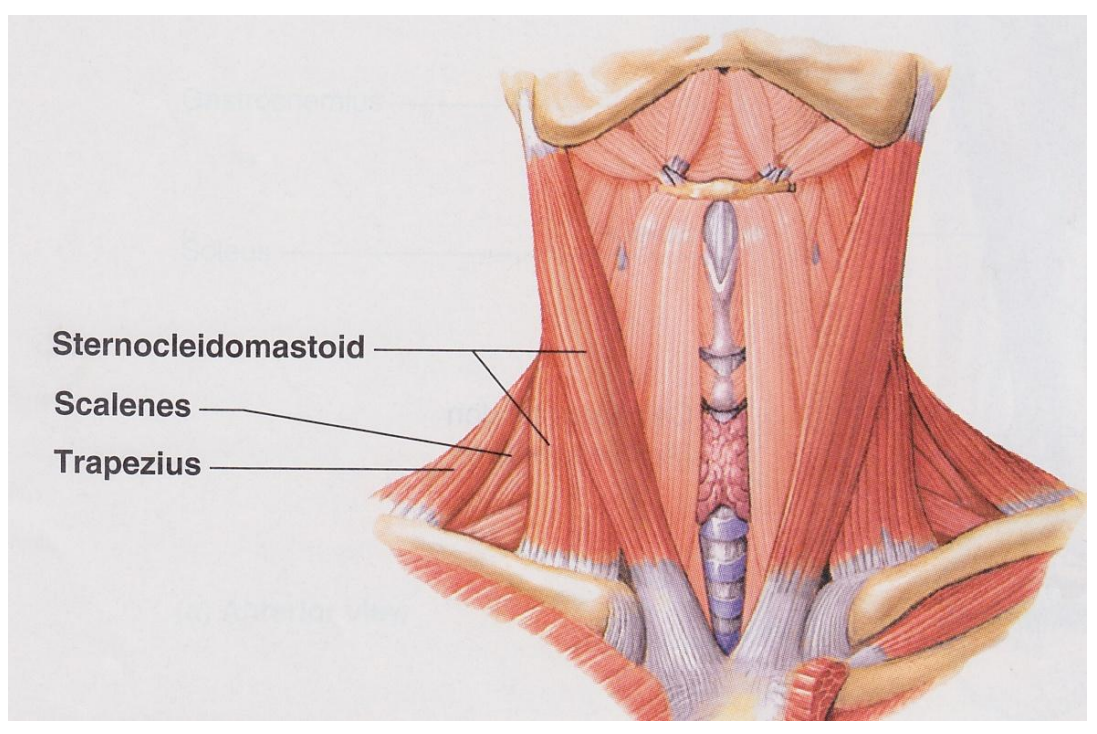

Figure 2.3: Neck Muscles (taken from [23]) 
Whiplash injuries are a major cause of abnormal rotation and excessive flexion of the neck. Examples of whiplash injuries include falls and vehicle accidents. Men's neck muscles are better developed compared to women [26]. Neck injuries can occur in two ways during whiplash. When passengers are struck from behind in a vehicle collision, the neck experiences hyperextension, as the head falls back, while the body is flung forward. The second scenario involves the head being flung forward due to rapid deceleration. This affects the cervical spine; one of the most flexible parts of the spine (C5-C7) [26]. Trauma can speed up the process of cervical spine degeneration, as the fluid filled cervical discs deteriorate $[23,26]$. These discs help with smooth neck movement and stabilization. Injuries pertaining to this area can lead to paralysis [27, 28]. Muscular dystrophic (MD) conditions (skeletal muscle degeneration) such as Emery-Dreifuss MD, Limb-Girdle MD (LGMD), Myotonic dystrophy (DM1), and Oculopharyngeal MD (OPMD) can also result in very weak neck muscles [27, 28].

While Emery-Dreifuss MD and LGMD may result in neck muscle weakness to a smaller degree, DM1 has the strongest impact on face and neck muscles [28]. The symptoms for this disease typical start to show between 20 and 30 years of age; however childhood onset can very well occur. Initial symptoms include a fatigue face and a thin neck. Resulting difficulties include breathing, swallowing, vision and cardiac complications

Apart from these specific conditions, neck pain can also result from muscle strains and from maintaining the same neck position for long periods. According to [26], two out of three people will suffer from neck pain at some point. Thus, it is evident that many are affected by neck conditions that hinder head stabilization and posture. Overtime, prolonged neck resistance may also start to obstruct one's breathing and limit his/her vision as well.

\subsection{Flailing and Jerky Movements}

\subsubsection{Jerk}

Jerky movements can occur during activities such as driving and amusement park rides. Sudden jerky motions cause, what is referred to as, flailing. The physics behind this chaotic 
motion is commonly referred to as "Jerky Dynamics" or "Newtonian Jerky Dynamics [29, 30]." A set of equations known as the jerk equations are used to represent this type of motion. Jerk can be computed by taking the third derivative of the displacement function, the second derivative of velocity or the derivative of the acceleration. The following formula can be used to compute jerk [29]:

$$
\vec{\jmath}=\frac{d \vec{a}}{d t}=\frac{d^{2} \vec{v}}{d t^{2}}=\frac{d^{3} \vec{r}}{d t^{3}}
$$

Where:

$\mathrm{t}=$ time

$\vec{a}=$ acceleration

$\vec{v}=$ velocity

$\vec{r}=$ position

Jerk can be felt as the change in pressure during a sudden increase or decrease in acceleration. In other words, during this motion, acceleration is not constant. Jerk is a very important motion to consider for the design of amusement parks, roller coasters, as well as motorized wheelchairs. Some patients, affected by distortions in different parts of the body, require a longer time period to react to changes in pressure, tension and stress. This can result in varying muscle reactions amongst patients. Figure 2.4 illustrates angular motion simulation profiles during motion across the y-plane.

\subsubsection{Computation of Jerk Systems}

Umut O. and Yasar S. use an algebraic system called the Genesio system to illustrate the derivation of jerk equations [31]. It consists of three equations with one quadratic term. The system is as follows [31]:

$$
\begin{gathered}
\dot{x}=y \\
\dot{y}=z \\
\dot{z}=-a x-b y-c z+x^{2}
\end{gathered}
$$



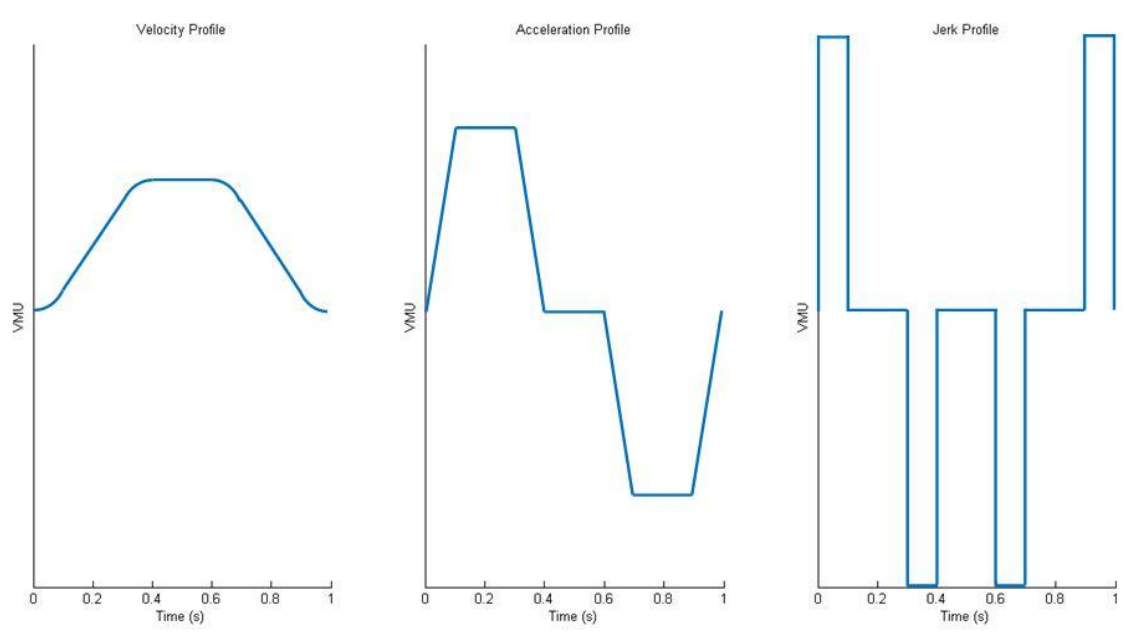

Figure 2.4: Angular Motion Profile of Simulator

The Genesio system was converted into jerky dynamics using both invertible and linear transformation. This specific system was found to be a simpler class of jerky dynamics with a larger range of parameter regions. This produced a very diverse set of dynamical behaviour.

Jerky dynamics can also be used to study instability caused by different forces. Wei Z. and Rongsheng W. U. studied instabilities caused by jerk by analysing the patterns of position, velocity and acceleration during rotational motions [30]. Unstable cases were found to have an exponential growth pattern amongst the variables. Results showed that instability is greater influenced by both absolute and planetary vorticity. Absolute vorticity deals with air, while planetary rotation is caused by the Earth's spin [30]. Overall, a periodic pattern was observed in position and velocity, when there were smoother changes in acceleration. Sudden changes in acceleration resulted in many shifts in direction. It was also found that changes to the initial vorticity may affect instability and set off chaos [30]. Thus, inertial motion predictions become difficult. 


\subsection{The Effect of Visuals}

It is expected that different people will take on different muscle reactions to sudden jerky motions. Further research can go into investigating whether other external factors can influence the muscle reactions. These factors may include the use of visuals, having a sturdy grip, and the direction/angle of motion. When it comes to the observance of visuals there are two distinct types: Earth-fixed and Natural chair-fixed [32]. Earth-fixed visuals are stationary and act as a reference target with respect to the Earth. Natural visuals move with the subject and are normally displayed on a screen fixed to the motion simulator. According to Lin J.J. et al., independent Earth-fixed visuals have been found to reduce both motion sickness and balance disturbances caused by simulators [32]. This is due to its ability to provide a sense of orientation that is similar to the vestibular receptors. Their study tested three different types of independent visual backgrounds. The first background used a grid, the second used a few clouds, and the third used many clouds. Clouds were used as they are perceived to be relatively stable [32]. The experiment involved exposing twelve subjects to motion through a driving simulator. Simulator sickness was measured using the Revised Simulator Sickness Questionaire (RSSQ). Results showed that the independent background with many clouds induced less nausea [32].

Similarly, Harris L. R., Dyde R. T., Jenkin M. R. compared the use of visuals and nonvisuals to depict the positions of the world [33]. Subjects were placed in a virtual environment and their perceived position of the world was tested by asking them to judge the displacement of a card. First, this card had movement that was phased fixed to the room. In order to test the effect of non-visuals, the subjects were moved in the dark and were asked to judge the movement of a floating object [33]. Accurate perception was evident when there was alignment between the card and the subject, and no parallax. It was concluded that both visual and non-visual cues were simultaneously necessary for position perception [33].

Lu W., Duh B. H., and Feiner S. came up with an alternative to very apparent cueing techniques for visual search [34]. The purpose of their study was to come up with a subtle cueing technique that improved visual search. They are many subtle cueing techniques available, such as change in visual details and contrast. This study used changes in contrasts in parts of the visual scene to obtain a subtle cueing effect. The scene included both still images as 
well as video images. The subjects were asked to find a cross target. The $12 \times 12$ pixels target was embedded within a $1024 \times 768$ pixels scene [34]. The reaction times and error rates were measured. Results showed that the using narrow congestion ranges can potentially replace explicit cueing. The overall pattern of the graphs showed that in both experiments, as the contrast levels increased both the reaction times and error rates were found to decrease [34]. The consistent pattern between the two also showed that results achieved from stilled images can also be applied to video images. Hence, the type and quality of visuals displayed affected the subjects' reaction times.

It was interesting to find many studies on the effect of angular motion towards the vestibulo-ocular reflex and head motions. A study by Xiang Y. and Yakushin S. B. investigated the different head tilts for varying frequencies [35]. The varying frequencies helped maintain manageable acceleration levels. The tests were performed on three monkeys. Results differed when the monkeys were on their side, as opposed to being upright. Peak vestibular-ocular reflex (VOR) gain changes were observed in the upright position. The overall pattern showed that during head oscillations with smaller angles in the forward pitch plane, there was a decrease in gain [35]. In order for maximal VOR gain change, the head was only able to experience small angles of rotation that were less than 15 degrees.

Although there have not been many studies that worked with abdominal or neck muscle activity and roller coasters, similar studies have examined virtual roller coasters, while others studied the relationship between EMG and balance.

\subsubsection{VR and Balance}

Shih Z. and team worked together to build a virtual roller coaster that simulated the real world experience found in theme parks [36]. The system consisted of a simulation software, motion platform, and control unit which were all brought together using VR techniques. The coordinate system consisted of an X-Y plane that represented the ground and the Z-axis that represented height [36]. Resistance, gravity, and friction were all considered when determining the speed and forces of motion. Two specific curves used in the track design included the spiral curve and the cubic Hermite spline curve. The hydraulic system was used for the motion platform, as it had the ability to provide greater power within a smaller volume. This made it 
easier to modify speed. Safety features included elbow rests and leg rails that separated the legs from the rotation frame [36]. The system successfully simulates roller coasters, while using minimal space and power. Future improvements that were mentioned include the ability to modify velocity and rotation in the $\mathrm{Z}$ axis.

E. Chang et al studied the effect of rest frames implemented in a virtual roller coaster [37]. This study focused on the effects of spatial perception. Rest frames were used to provide the user with stationary objects, helping him/her select a rest frame, which as a result would reduce cybersickness. Two subjects participated in the study to test the rest frame and the nonrest frame conditions. The study showed that the participants experienced lower levels of motion sickness during the rest frame condition [37]. This was further verified by a difference in the EEG readings between the rest frame and non-rest frame conditions.

A study was performed by Ma S. et al to analyze muscle activity during specific tasks via VR [38]. This paper brings out the importance of EMG feedback in hand grasping and rotation motion rehabilitation. The VR environment provided interaction between the user and virtual objects. The integration of an EMG feedback system allowed the analysis of hand motions and their stages of improvements throughout the training process. The M3400 BIOPAC EMG system was used to detect muscle activity [38]. The system was incorporated into a VR system using the DATA TRANSLATION DT9816 [38]. The entire system was very useful in measuring muscle fatigue, tension, net force and activation. The system was tested on 10 participants. Results have proven to show an improvement in the skills trained via the EMG embedded VR system [38].

The EMG activity of various muscles, including gastrocnemius, tensor fasciae latae, and semimembranosus was analyzed during a balance test using VR, by Cikajlo I. and team [39]. The effectiveness of telerehabilitation was analyzed by the use of a haptic floor. When the haptic floor was in effect, there was greater muscle activity seen as the distal and ankle muscles worked to stabilize the tibia. The study concluded that incorporating a haptic floor in balance training systems can greatly enhance postural response and the overall training experience [39].

Moreover, a study performed by Ando T., et al. studied the primary muscles involved in rolling over [40]. This study was used to develop a support system for cancer bone metastasis 
patients having trouble rolling over. The EMG signals of four subjects were measured by placing four markers on the right and left acrominon and anterior superior iliac spine. Electrodes were attached to the erector spinae, rectus abdominis, internal abdominal oblique, and the external abdominal oblique. The study went on to show that the internal abdominal oblique muscles were active during the early stages of roll-over motion [40].

Mixed reality sickness occurs due to the sensory confusion between the real and virtual worlds [41]. A study regarding mixed reality sickness was performed by Nakajima S., et al. This paper analyzes the motion after-effect (MAE) theory to determine whether it provides a useful evaluation of motion sickness and the effect of auditory stimuli. Eight subjects aged 2141 participated in the study [41]. Equipments such as a function generator and a human shaker helped oscillate the seated subjects. The MAE stimulus was created by displaying two black horizontal stripes on the sides of the screen and were moved at a constant speed in opposite directions. Results showed that MAE is indeed a useful evaluation method for motion sickness. The study also found that auditory stimulation helped reduce motion sickness [41].

Hence, it is evident that the type of visuals portrayed also affect human reaction. The effect of visuals on EMG signals can also be tested during various rotational movements. Since human perception and balance are affected by visuals, the muscle reactions may also be affected. These factors would need to be considered when designing VR rehabilitation training programs.

\subsection{Whiplash}

Whiplash injuries can be caused by car accidents, amusement park rides, and falls. According to [26], millions of Americans are affected by whiplash each year. Depending on the severity of the injury and the individual, recovery time will vary from weeks to years. Out of the millions affected, $10 \%$ become permanently disabled [26]. Wheelchair users are mostly susceptible to these types of injuries through falls that occur during patient transfers. Chronic pain resulting from previous injuries can affect muscle behaviour, predominantly in the neck. One simple example where neck muscle strains can affect muscle behaviour is when wheelchair users travel up and down ramps. Most wheelchairs have a headrest to help with sudden 
backward tilts, but forward motions require additional work from the neck muscles to help stabilize.

Recent research has shown that the first reaction the body has to linear jerk is trunk movement [26]. A forward jerk causes the trunk to push forward, resulting in a backward neck motion. This reaction is referred to as the S-phase, as the upper vertebrae forms an S shape. This phase is when most injuries occur, as the vertebrae is stretched in two opposing directions. Patients with previous injuries may also have unnatural neck muscle behaviour [26]. This occurs when patients keep a very rigid posture to avoid further pain be inflicted on the same area. This action is referred to as muscular bracing and can lengthen the recovery time. Interestingly enough, research studies have come to show that cervical collars, in fact, hinder recovery, rather than aiding it [26]. There are many neck exercises that help with post injury neck pain. Severe cases also have the option of using electric current to reduce pain caused by specific nerves, a process referred to as radiofrequency neurotomy [26].

There have been many studies done to analyze neck motion during whiplash and postwhiplash injuries. Grip $\mathrm{H}$. and his team study various different neck movement patterns of patients affected by whiplash, as they tried to classify the different types using neural networks [42]. Three dimensional motion data acquired from 59 subjects were fed into the back propagation neural network. Data reduction was performed using principle component analysis, and the accuracy was able to be increased to $89 \%$ [42]. The subjects in this study were asked to perform four different head movements, including flexion, extension, and rotation to the left and right. Markers were placed on the subjects' head, and three dimensional data was recorded using a ProReflex system. Some of the parameters computed were angular velocity, neck range, mean, and reaction time. Velocity and range of neck motion was found to be good indicators of neck condition severities. This study did not have the subject undergo rotational movements and another way to further build on this study would be to compare the neck movement to body movement in patients affected by whiplash. In a previous study by the same team, they were able to find a significant difference in angular velocities and mean values between the healthy subject and those affected by WAD (whiplash related disorders) [42]. 
Ehtemam F. et al. also studied neck motions and how they are affected by galvanic vestibular stimulation (GVS) [43]. GVS uses electric currents to modify vestibular information. It is believed that head motion can be minimized with the use of impulse and sinusoidal forces. This study was performed on the SCM muscles of 11 subjects. A motion capture system was used to measure head and torso kinematics. Subjects were seated and the duration of the tests lasted $80 \mathrm{~s}$. When higher frequency stimuli (greater than $1.2 \mathrm{~Hz}$ ) were present, the subjects were able to perceive head movement [43]. As the amplitude of the stimulation increased and the frequency decreased, the response magnitude was found to increase.

Hynes L. M. and Dickey J. P. studied head kinematics in rear-end collisions [44, 45]. The focus of their study was to find out whether a change in jerk or acceleration affected head movements. A robotic platform was used to exhibit different levels of acceleration and jerk. This study used linear motion from the posterior to anterior direction. Subjects were seated in the passenger seat of a Honda Accord that was mounted to the robotic platform. The peak accelerations ranged from $5.51 \mathrm{~m} / \mathrm{s}^{2}$ to $10.78 \mathrm{~m} / \mathrm{s}^{2}$, while peak jerk ranged from $267 \mathrm{~m} / \mathrm{s}^{3}$ to 380 $\mathrm{m} / \mathrm{s}^{2}$ [44]. Experiments were performed on 21 subjects. Surface electrodes were placed on the TRAP, splenius capitis, and SCM muscles. Results showed that forehead acceleration is greatly affected by both jerk magnitude and platform acceleration. This study can be further built on by considering how rotational motions affect the neck. Another interesting aspect to consider would be how the neck and body motions differ, during jerk.

Yang C. et al. analyzed the smoothness of neck movements between healthy individuals and patients with neck disorders [46]. The test group consisted of 18 healthy individuals and 18 patients with mechanical neck disorders (MND). In this study subjects were asked to perform neck motions, including flexion, extension, and rotations. Kinematic data was processed using a fourth order Butterworth filter, with a cut-off frequency of $6 \mathrm{~Hz}$ [46]. The linear accelerations and peak tangential velocities were determined from the data. The spectral entropy was computed from the power spectrum using the following formula [46]:

$$
S p E=\frac{-1}{\log _{10} M} \sum_{i=1}^{n}\left[P S D_{i} \times \log _{10}\left(P S D_{i}\right)\right]
$$


In equation 2.5 PSD represents the power spectrum density values, and $\mathrm{M}$ represents the number of frequency bins. Statistical analysis was also performed on the kinematic data. Subjects with neck pain showed longer durations of movements and higher spectral entropy. However, there was no significant difference in tangential velocity. The unsmooth neck cervical movements in patients with MND are caused by attempts to maintain spine stability and avoid further pain inflictions.

The EMG and kinematic responses of the neck were studied during changes in acceleration and jerk, by Siemund G. P. and Blouin J. [47]. Experiments were performed on 23 subjects under eyes open and eyes closed conditions. Activity from the SCM, SCAL and cervical paraspinal muscles were analyzed. They hypothesized that if increased jerk stimulus resulted in solely higher head kinematics and no change in muscle response, this would indicated that jerk may increase causes of whiplash injuries. But, if increasing jerk had no effect on head movement and muscle activity, this would indicate that jerk played a small role in whiplash injuries. EMG signals were bandpass filtered from 20 to $500 \mathrm{~Hz}$. Subjects were seated in a car seat with the head rest removed, in order to fully measure floppy responses of the head. The seat was mounted to a sled that was powered by linear induction motors. Peak linear acceleration and peak angular acceleration of the head were determined from the kinematic data. RMS values of the EMG signals were computed using a $20 \mathrm{~ms}$ window. Results showed that the SCM and SCAL muscles were most active during higher amplitudes in the forward perturbations, while the paraspinal muscles were most active during high amplitudes of backward perturbations [47]. Head angles were found to increase under the eyes closed condition. Sled acceleration was found to play a greater role in kinematic responses, as opposed to jerk. Hence, this paper argues that jerk plays very small role in whiplash injuries.

\subsection{Previous Experimentation}

Previous related experiments were performed at Ryerson, by Shafeie M., using a spherical VR motion simulator [48]. The study focused on abdomen and back muscle behaviour. This simulator was not a fully enclosed environment. Results from this study showed that abdomen and back muscles were most active during forward rotations and trials with VR 
present. Furthermore, maximum and minimum muscle contractions of $31.8 \%$ and $3.66 \%$, relative to MVC were achieved, respectively [48]. The study also found that abdomen and back muscles had higher activity during higher speeds of motion. The main cause of neck pain and injuries is the large difference in speed and direction between the neck and torso. This thesis will build on the previous experiments, by comparing neck muscle behaviour, as well as head and body motion, under similar conditions, using a fully enclosed motion simulator.

\subsection{Chapter 2 Summary}

It is evident that there are many neck conditions that can affect posture and muscle contractions. There have been many studies performed that analyze head and neck kinematics along with neck muscle EMG signals during jerk motions. However, there have not been many studies that have looked into neck activity during rotational motions. This thesis focuses on rotational motions and aims to examine how body and neck motions work together to stabilize posture. 


\title{
Chapter 3
}

\section{Experimental Setup and Methods}

\author{
7 HIS study was approved by Ryerson University's Research Ethics Board. All subjects \\ 1 were asked to sign a written consent form, prior to the performing the experiments. The \\ eligibility forms gathered additional information such as height and weight, which was also used \\ during analysis. Data was acquired from 20 healthy subjects between 18 and 35 years of age. \\ The participants had no history of falls or neck difficulties, and no somatosensory, vestibular, \\ cognitive or musculoskeletal impairments.
}

\subsection{Subject Preparation}

Subjects were asked to wipe the skin with alcohol wipes and wait until the skin was dry before the electrodes were placed. This helped remove any dirt or oil that tends to affect electrode-toskin contact, and as a result generate noise in the signals. When measuring EMG, voltages differentials are measured from 2 electrodes per muscle. Neck muscle activity was recorded by placing electrodes on the left and right SCM, SCL, and TRAP muscles, totalling 4pairs of electrodes on the front of the neck and 2 pairs on the back of the neck (as seen in Figures 3.1 and 3.2). The neck has a strong pulse, as it is very close to the heart. Hence, EMG data recorded in the neck tends to be affected by ECG crosstalk. In order to remove ECG crosstalk, one would need sole ECG data as reference $[49,50]$. For that reason, 2 additional electrodes were placed on the chest ( $3 \mathrm{~cm}$ below the left and right clavicles), to record ECG data, simultaneously. Elbows were used as ground, during acquisition. The first accelerometer (GreatLakes NeuroTechnologies, USA) was placed on the back of the head where the parietal and occipital 
bones meet (see Figure 3.3). The second sensor was placed on the torso near the upper rectus abdominis muscle. The neck EMG, neck movement, body movement, and ECG were measured simultaneously. Once the snap leads were connected to all the electrodes, the cables were fed into the BioRadio user unit. Loose cables were twisted and taped to the subject using medical tape. This prevented motion artifacts from occurring in the EMG signals. The radio was connected to the PC via USB Bluetooth adapter.

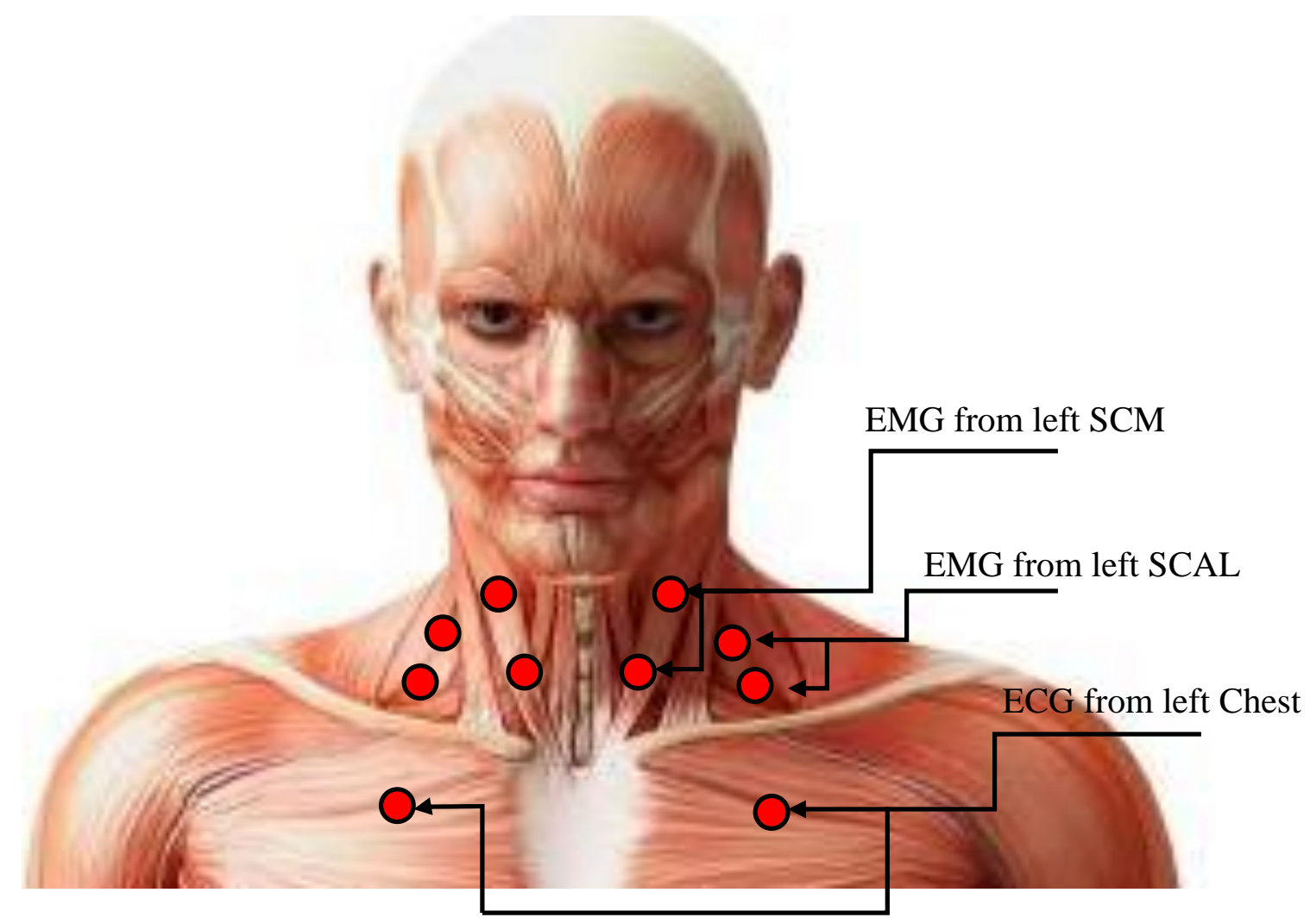

Figure 3.1: Anterior Electrode Placement (adapted from [51]) 


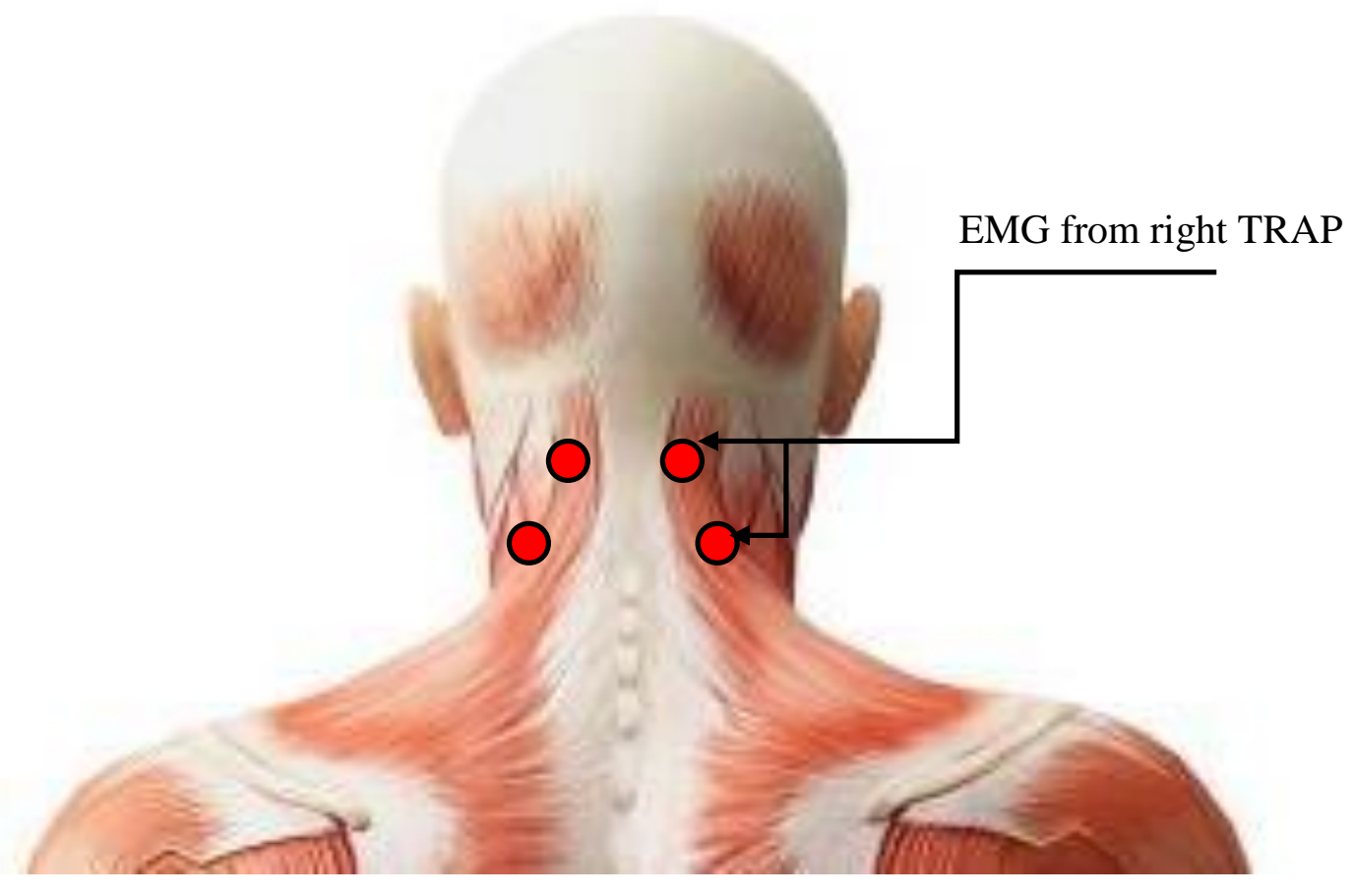

Figure 3.2: Posterior Electrode Placement (adapted from [51])

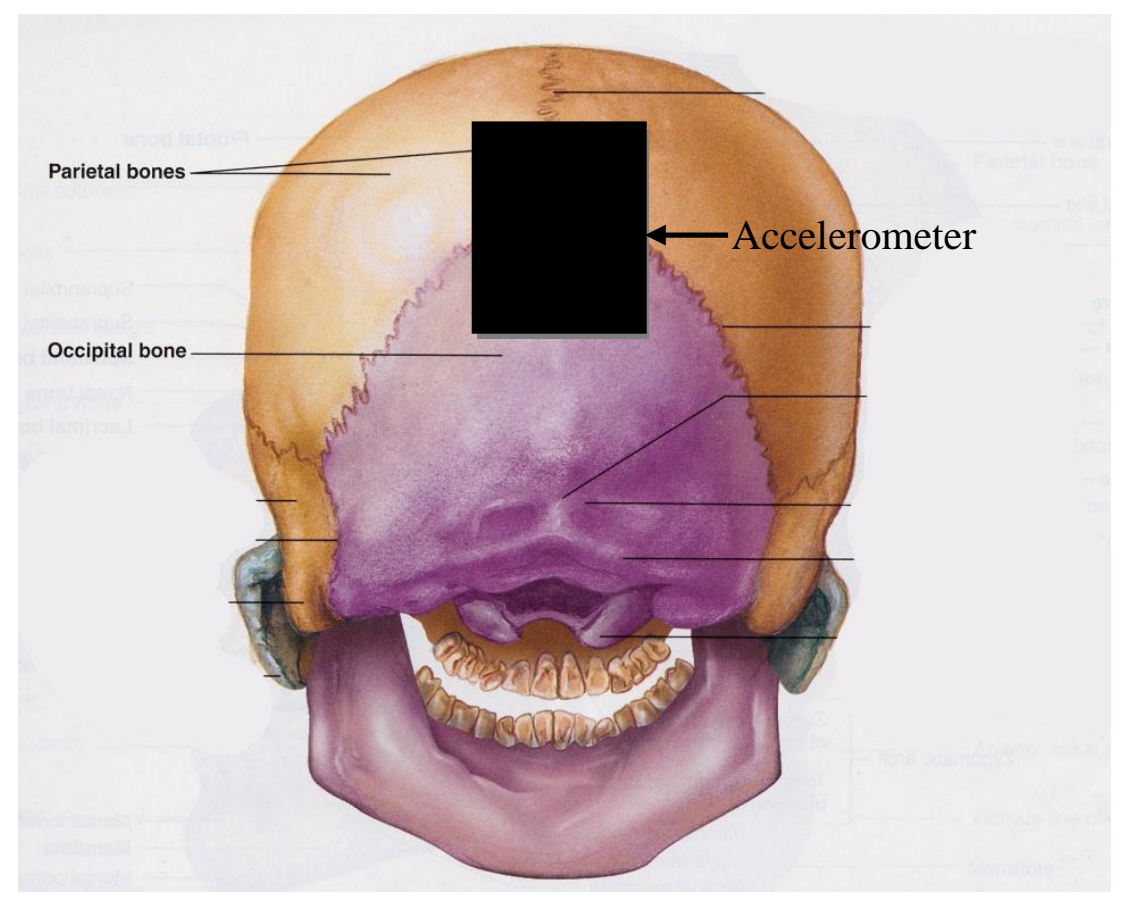

Figure 3.3: Accelerometer Placement (taken from [23]) 
Once the setup was complete, MVC for each neck muscle was recorded for the forward and backward rotations. This was done by first asking subjects to move their head all the way to the back as far as possible, while fully contracting their neck muscles. Once the backward motion was recorded the subject was asked to repeat this for the forward motion. Three trials were recorded for each direction. After the completion of MVC recordings, the subjects were seated inside the simulator. The waist belt was fastened, followed by the shoulder harness. The initial set of experiments were conducted with the projector off, where the subjects were in complete darkness (for no visuals). The canopy was closed, and the simulator was stabilized and raised. The subjects were rotated 45 degrees in the forward and backward pitch planes, 3 times each, under complete darkness. The 3 trials acquired for each direction of rotation were randomized. The travel time from neutral to 45 degrees in either direction took approximately 1 second. The PC was set to record right when the simulator was released for movement and the recording was stopped 1 second after the stop of motion (totalling 2 seconds). This allowed to gather some after effects of how the neck muscles behaved for a short period of time after motion was complete. The simulator was brought back to neutral position after each direction of motion.

Once this set of experimentation was complete, the simulator was lowered, and the canopy was opened to turn the projector on (for visuals), as the subject remained seated. The visuals displayed consisted of a tunnel moving in the forward direction. The MaxFlight logo displayed near the center of the screen acted as a focal point. Figure 3.4 shows an image of the display during motion. Once again, the canopy was closed, the simulator was stabilized and raised to perform the second set of experiments. Again, the subjects were rotated 45 degrees in the forward and backward pitch planes, three times each. Once the second set of experiments (with visuals) was complete, the simulator was lowered, the canopy was opened, the seatbelts were released, and the subject was helped out of the simulator. the cables were disconnected from the electrodes, the electrodes were removed from the body and were disposed. 


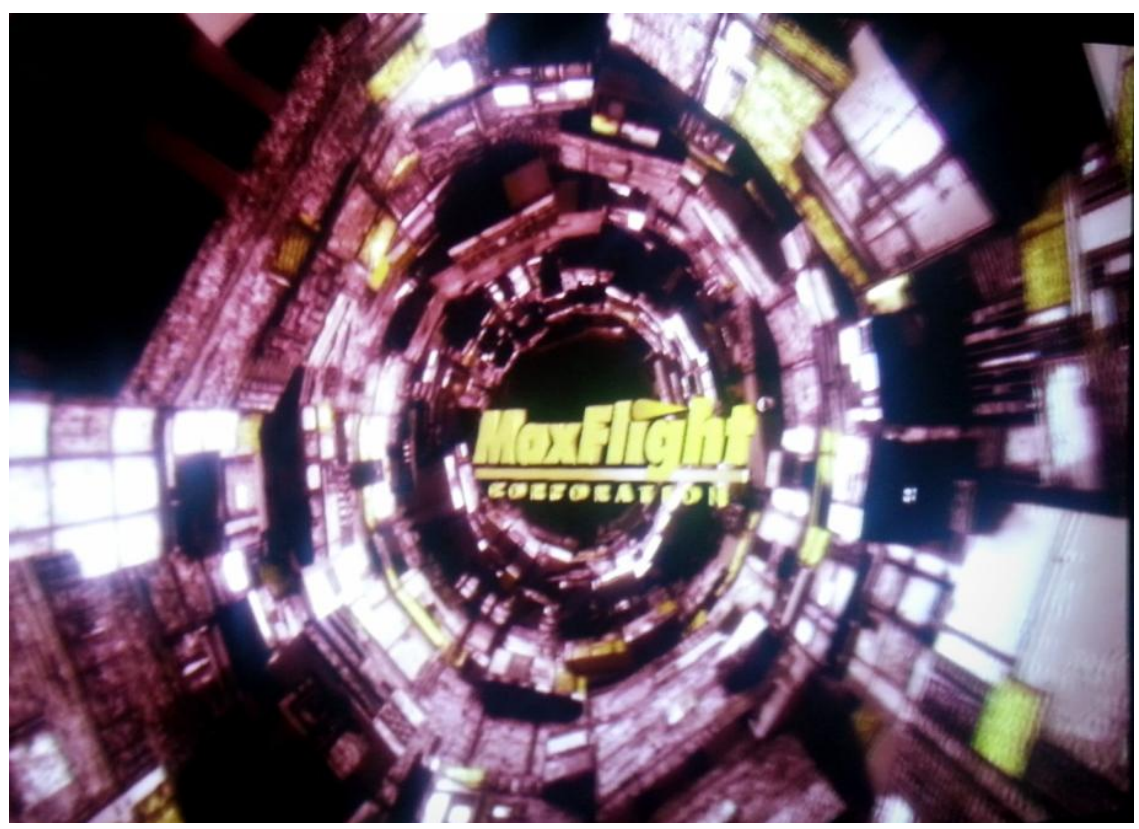

Figure 3.4: Visual Display of Tunnel Moving Forward

\subsection{MaxFlight Motion Simulator}

The Maxflight motion simulator was used for the experiments. This interactive simulator has full 360 degrees of motion across the pitch (y) and roll (x) axes. The COR for the pitch plane is located behind the seat. The computer driven simulator has preprogrammed rides and games, but can also be manually rotated to desired pitch and roll angles. Experiments consisted of 45 degree rotations (with and without visuals) in the forward and backward pitch planes. Figure 3.5 shows a labelled diagram of the three dimensions of motion, while Figure 3.6 shows subject placement relative to the COR. The distance from the subject's center of mass to the COR is approximately $0.84 \mathrm{~m}$. Thus, the subject travelled a distance of $0.66 \mathrm{~m}$, as they were rotated $45^{\circ}$.

Focusing on a specific object ahead can greatly affect neck muscle responses. According to Szabo et al., one of the important factors to consider when using visuals during balance and posture tests is the focal point [53]. Hence, the visual scene displayed portrayed depth (in the form of a tunnel) and a logo towards the center. This allowed subjects to focus towards the centre of the screen, while experiencing the subtle illusion of travelling forward through a tunnel. When testing without visuals, the projector was switched off and the subjects were in complete 
darkness, once the canopy was closed. Table 3.1 summarizes the simulator dimensions and Table 3.2 shows the design specifications. Photos of the simulator can be found in Figures 3.7-3.10.

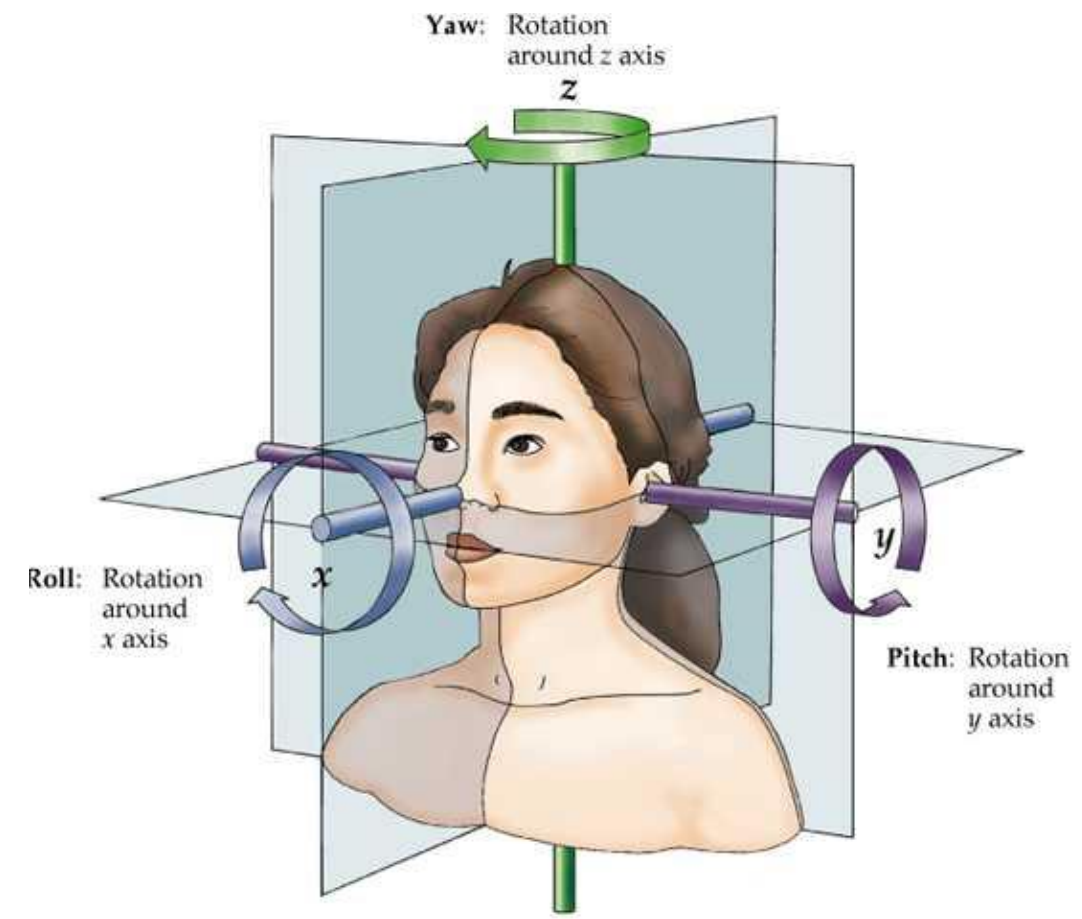

Figure 3.5: Three Dimensions of Motion (taken from [52])
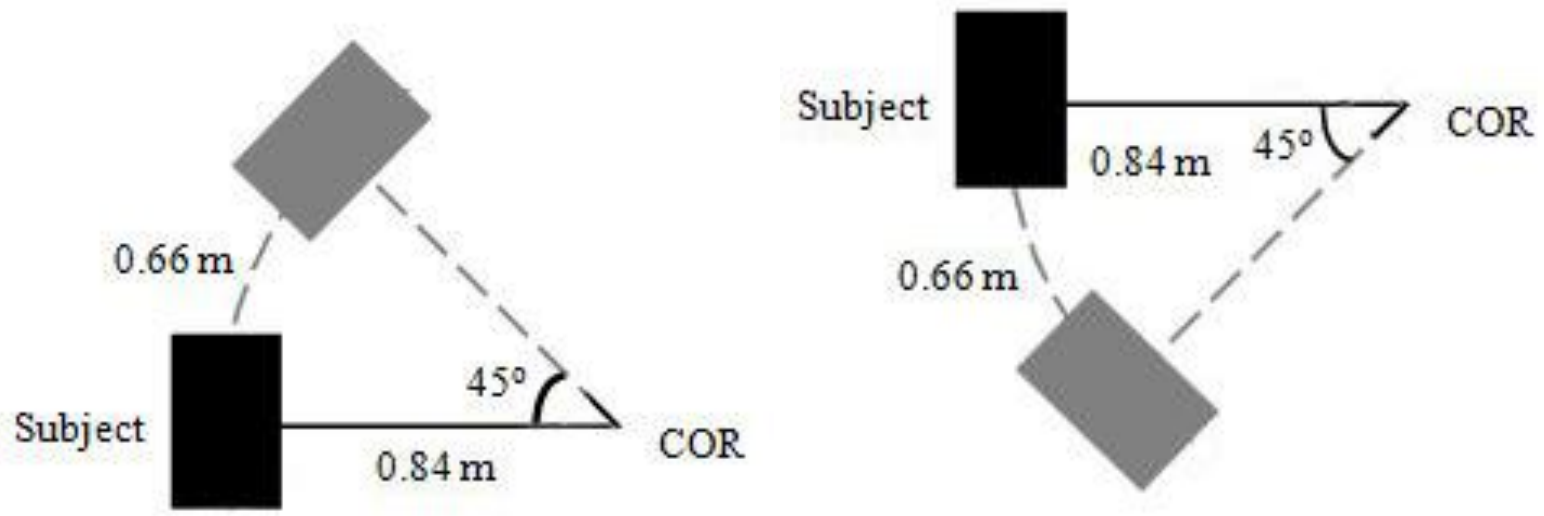

Figure 3.6: Subject Rotated in the Backward Pitch Plane (left) and Forward Pitch Plane (right) 
Table 3.1: Maxflight Simulator Specifications

\begin{tabular}{|l|l|}
\hline Height & 12 Feet, 9 Inches (3.88 meters) \\
\hline Width & 12 Feet, 4 Inches (3.76 meters) \\
\hline Length & 18 Feet, 5 Inches (5.62 meters) \\
\hline Weight & 4000 lbs. (1814.36 kg) \\
\hline
\end{tabular}

Table 3.2: Experimental Design Specifications

\begin{tabular}{|l|l|}
\hline Range of Motion & 45 degrees \\
\hline Speed & 45 degrees/second \\
\hline Visual Display & Included motion, depth, and a focal point \\
\hline
\end{tabular}
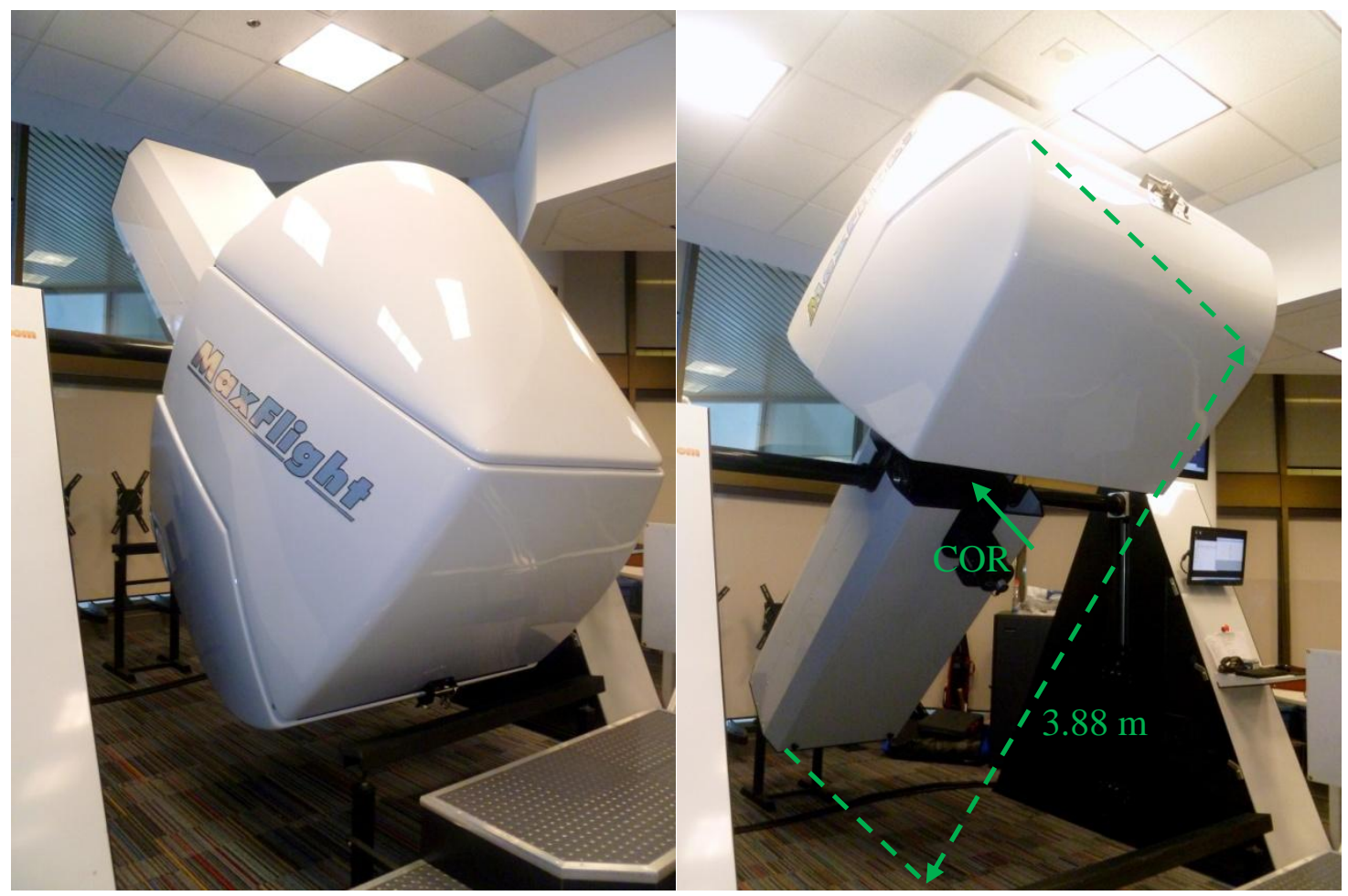

Figure 3.7: Motion Simulator at 45 degrees forward (left) and 45 degrees backward (right) 


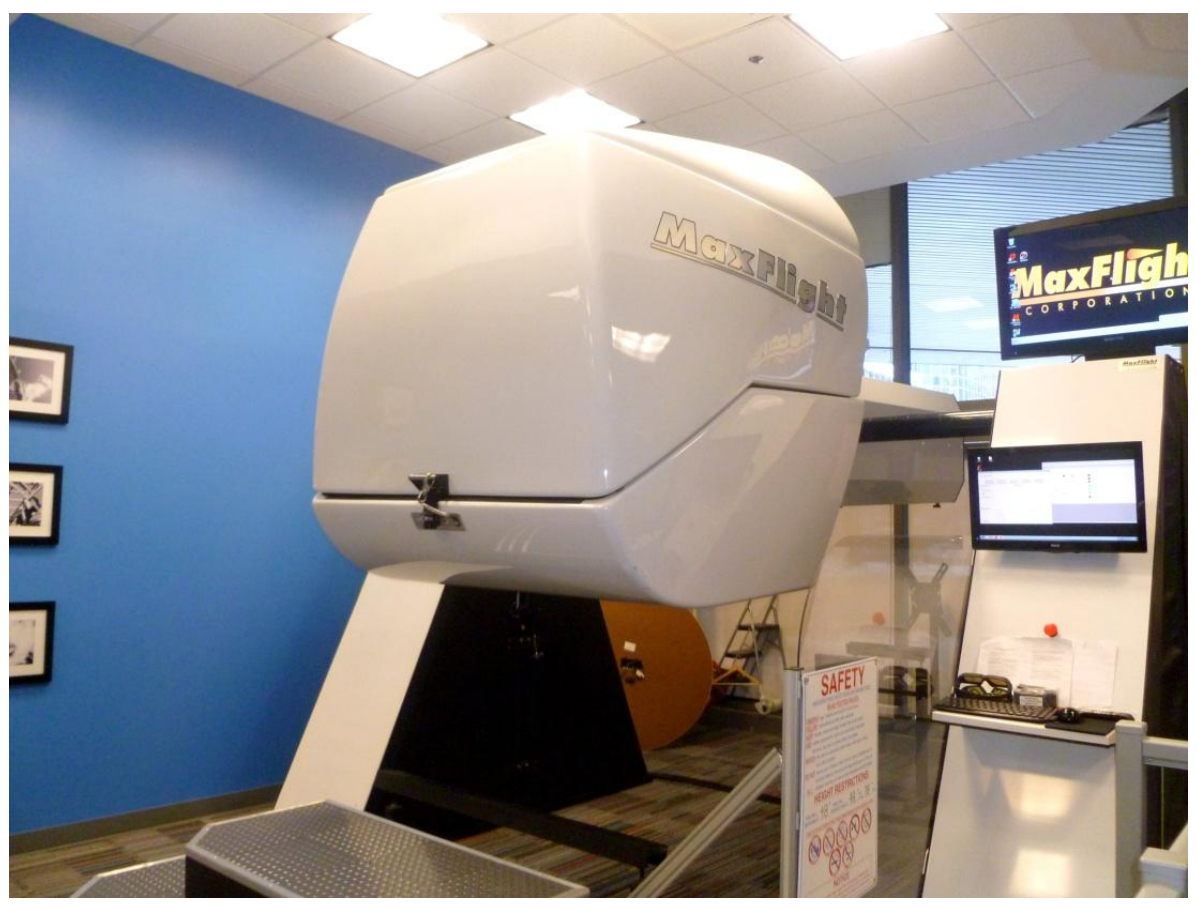

Figure 3.8: Motion Simulator at Neutral Position

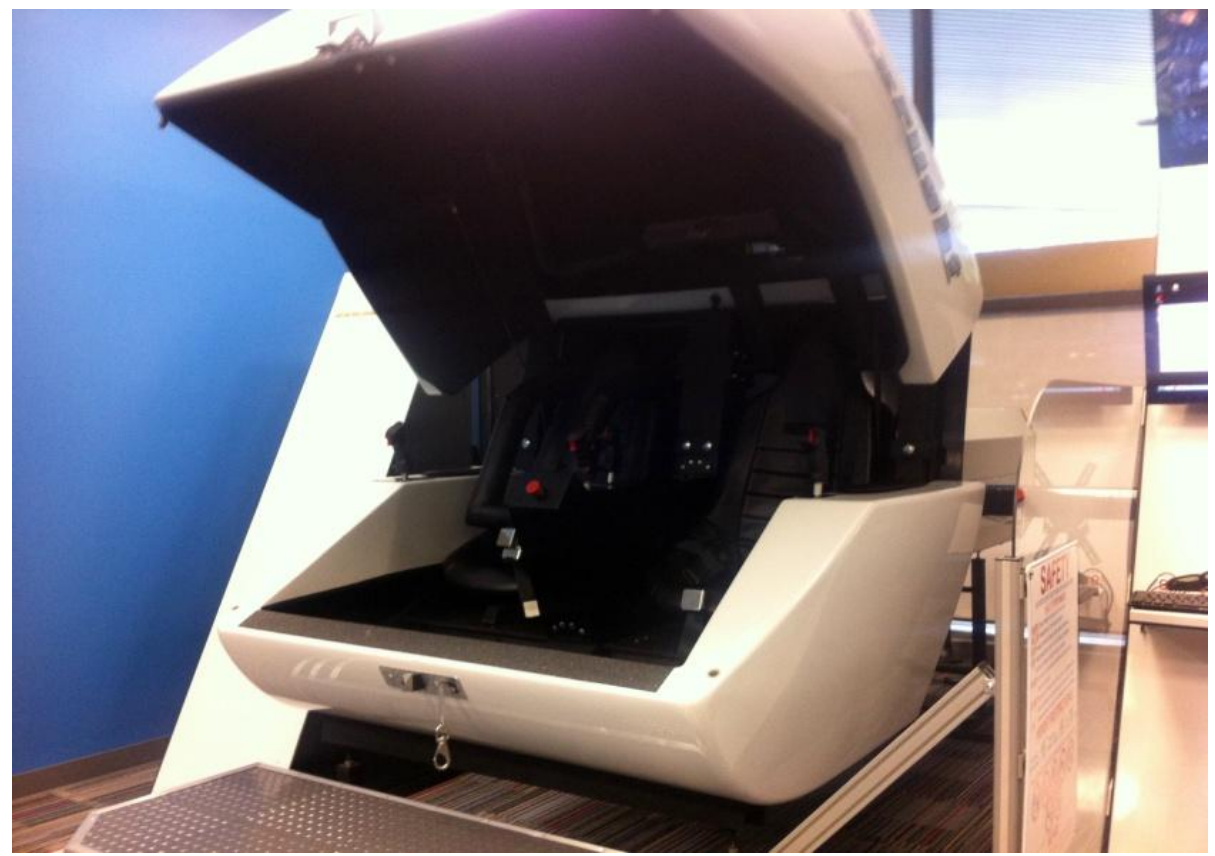

Figure 3.9: Motion Simulator with Canopy Open 


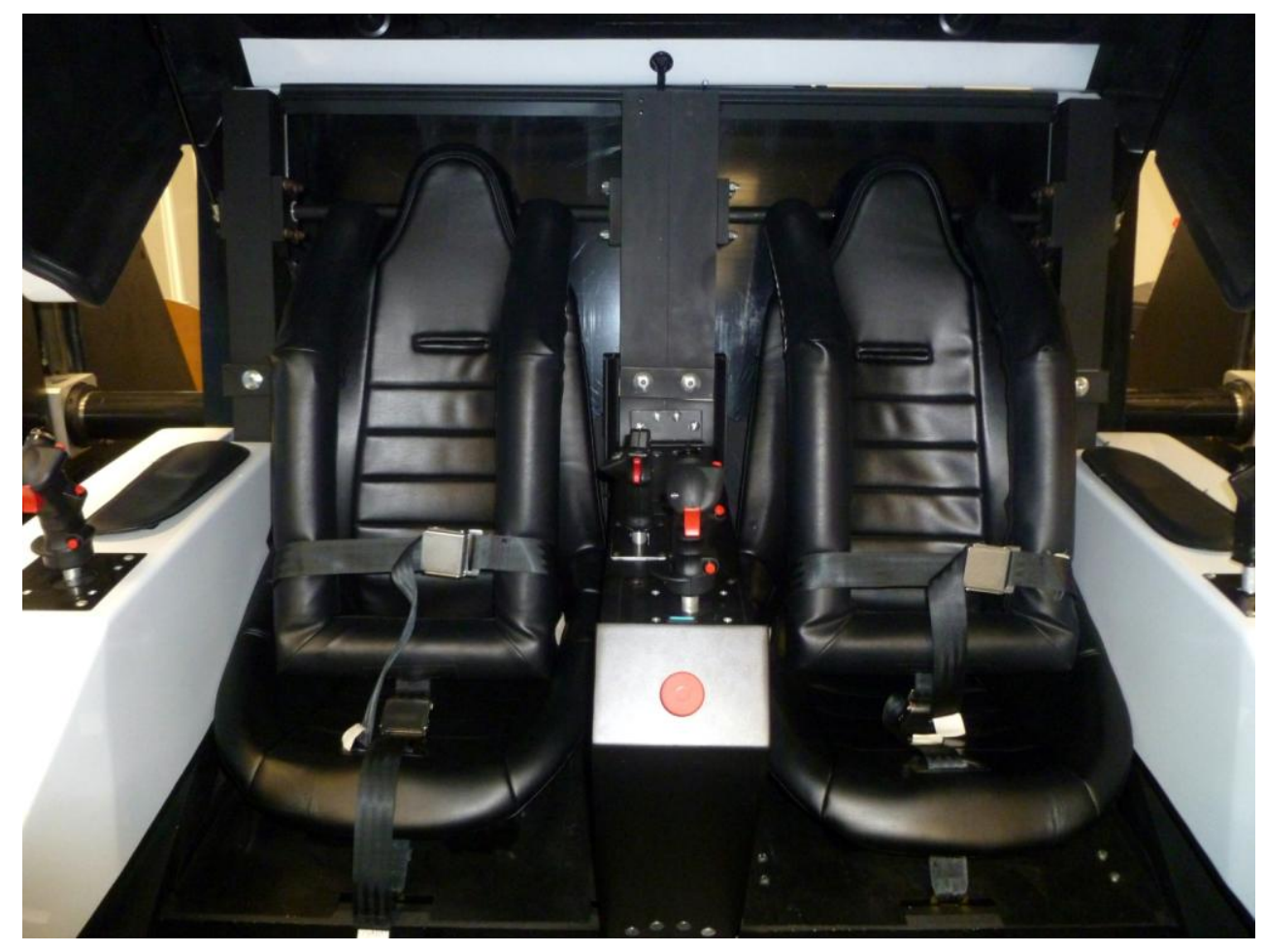

Figure 3.10: Interior of Motion Simulator

The simulator was prepared by running through the daily checklist, to make sure all parts were secure and working properly. Once the checking was complete, the simulator was set to go through a test ride, with no subjects inside.

\subsection{BioRadio}

The wireless BioRadio was used to acquire EMG, ECG, and accelerometer data from the subjects. This programmable device has 8 channels per radio, a transmission range of 100 feet, and a 2.4-2.484 GHz radio frequency band. The input range of the device is $\pm 1 \mu \mathrm{V}$ to $\pm 2 \mathrm{~V}$. The input impedance of the device is $500 \mathrm{M} \Omega$ and filter input bandwidth ranges from $0.5 \mathrm{~Hz}$ to 250 Hz. Data can be recorded at a sampling rate of up to $16 \mathrm{kHz}$. For this study, a sampling frequency of $1000 \mathrm{~Hz}$ was used, as many reputable sources would agree that a sampling rate of $1000 \mathrm{~Hz}$ would be ideal $[54,55,56]$. 
Accelerometer data was measured using the embedded motion sensor. This sensor records both tri-axial linear acceleration and angular velocity. A diagram of the radio, as well as the axes and directions can be seen in Figure 3.11.

The BioRadio system along with 3M Red Dot $\mathrm{Ag} / \mathrm{AgCl}$ electrodes were used for data acquisition. These wet electrodes are commonly used in clinical settings, as they are easy to apply, have low weight, and are disposable [58]. However, these wet electrodes do have their share of disadvantages. They tend to dry out due to dehydration, and as a result have a limited shelf life. Long term continuous measurement may result in loss of contact, causing noise and contact impedance. A detailed study was performed relating to the differences between wet and dry electrodes for medical signal acquisition [49, 59]. Results showed that although dry electrodes had a higher signal-to-noise (SNR) ratio, the wet electrodes produced results with a lower standard error. For the purpose of this experiment, wet electrodes were used, as the subjects were only required to wear them for a short period of time.

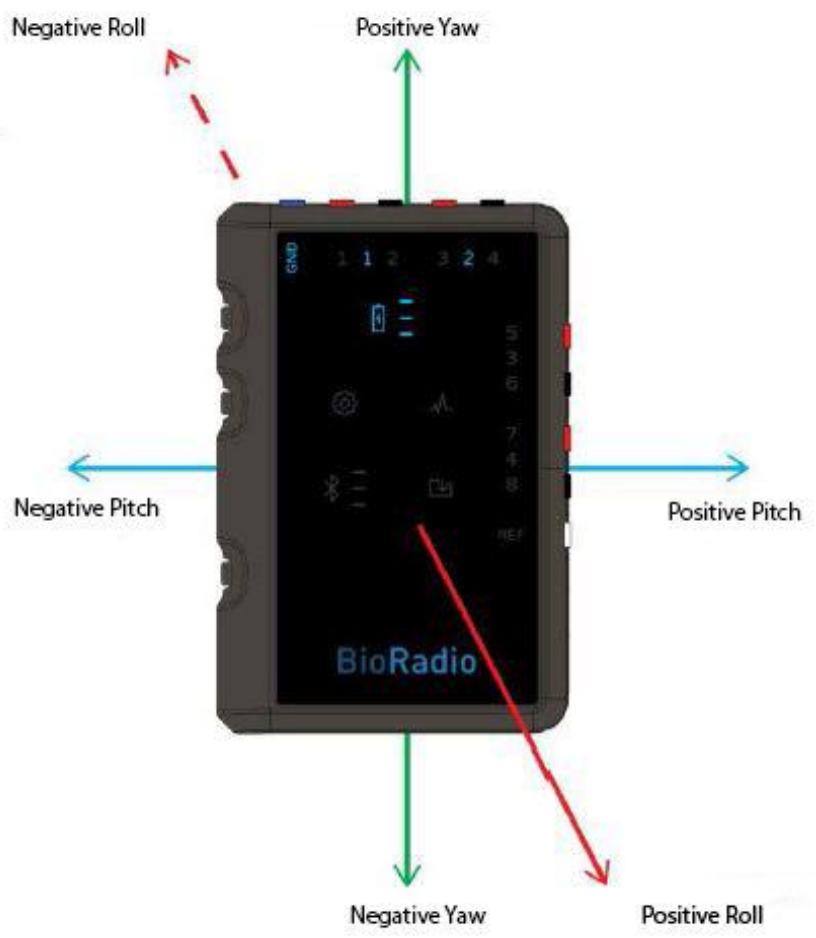

Figure 3.11: BioRadio Axes (adapted from [57]) 


\subsection{Algorithm}

The BioCapture recording (BCR) files were converted into comma-separated values (CSV) files, so that they can be opened in MATLAB to perform signal processing.

\subsubsection{Adaptive Filtering}

The first difference that was noticed during testing stages, between EMG signals acquired from neck muscles versus truck muscles was ECG crosstalk. A strong pulse can be detected in the neck, with bare fingers. ECG crosstalk can show up in areas closer to the heart. The signal resulting from blood flow is the stronger near the heart, as it pumps blood out to the body. The strength of the signal then dissipates as the blood travels away from the heart to all parts of the body. Since the neck is closer to the heart, ECG crosstalk was observed in the EMG signal.

Adaptive filters use the input signal and the associated noise signal to adjust the frequency and impulse responses. This technique has been popularly used to distinguish ECG signals between the mother and fetus [24]. The adaptive filter takes in the signal and associated noise as inputs. The noise $\mathrm{e}(\mathrm{n})$, is estimated based on a reference input, and is removed from the original signal. Another example application is for wearable medical devices. Noise and motion artifacts caused by muscle signals (EMG), were successfully removed from ECG signals acquired from devices intended for continuous, daily monitoring [49]. As far as EMG processing goes, motion artifacts and interferences are commonly removed using wavelet transformation. Here, the adaptive filtering method is used to remove ECG interference from EMG. When implementing this technique, the reference input $r(n)$, should be a signal that relates to the noise looking to be removed. In this case, the reference was an ECG signal acquired simultaneously from the chest, during the experiment. Equation 3.1 represents the output of the adaptive filter [24].

$$
y(n)=\sum_{k=0}^{N-1} \omega_{k} r(n-k)
$$


The adaptive filter output consists of $\omega_{k}$ which represents tapped weights, $N$ which is the filter order, $r(n)$ which is the input, and $k$ which is the tap number. The following equation is used to compute the weight vectors:

$$
w(n+1)=w(n)+2 \mu e(n) r(n)
$$

In Equation 3.2, the convergence parameter is represented by $\mu$ and the error estimation is represented by $e(n)$. Figure 3.12, shows a block diagram of the filter design.

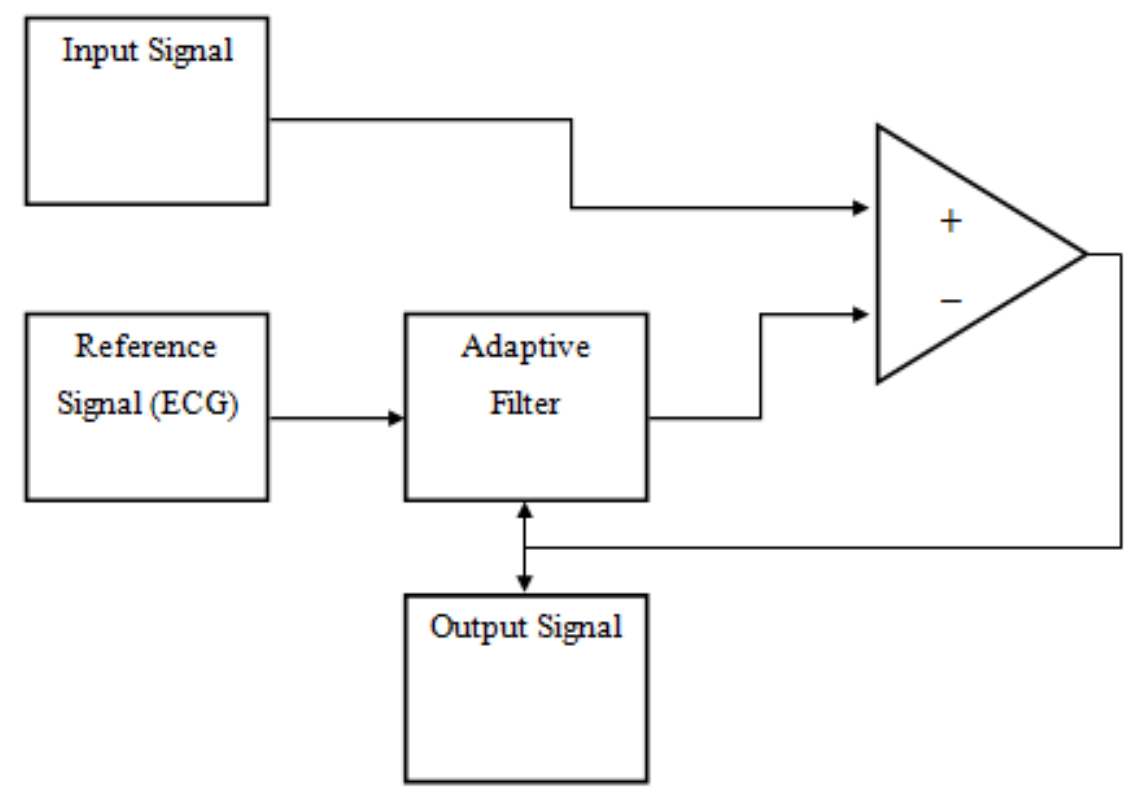

Figure 3.12: Adaptive Filter Block Diagram (adapted from [24])

The ECG data was acquired at the same frequency as the EMG signals. When comparing the EMG and ECG signals, the pulse in the EMG signals were lined up with the ECG signal. However, the ECG signals acquired from the chest, had a slightly larger magnitude. This is expected, as the heart signal from the chest is much stronger than the neck. Hence the ECG signal was scaled down about 2.5 times, to match the magnitude of the pulses observed in the EMG signal. 


\subsubsection{EMG Signal Processing}

The EMG signal measures the electrical impulses of muscles [24]. They can be used to detect muscle spasms, fatigue and conditions, such as carpal tunnel syndrome. When it comes to signal processing of EMG signals, the bandpass frequency ranges from $10 \mathrm{~Hz}$ to $20 \mathrm{~Hz}$ and 500 $\mathrm{Hz}$ to $1000 \mathrm{~Hz}[24,60]$. The bandpass filter consists of both a high-pass and low-pass filter which help eliminate low frequency artifacts and high frequency artifacts, respectively [24]. Muscle activity is represented by action potentials on the EMG and the fluctuating frequencies and magnitudes depend on the strength of contraction. Abnormalities can be detected using unusually high or low amplitudes, as well as frequency and duration of the action potentials. The block diagram below depicts the signal processing steps executed in this thesis.

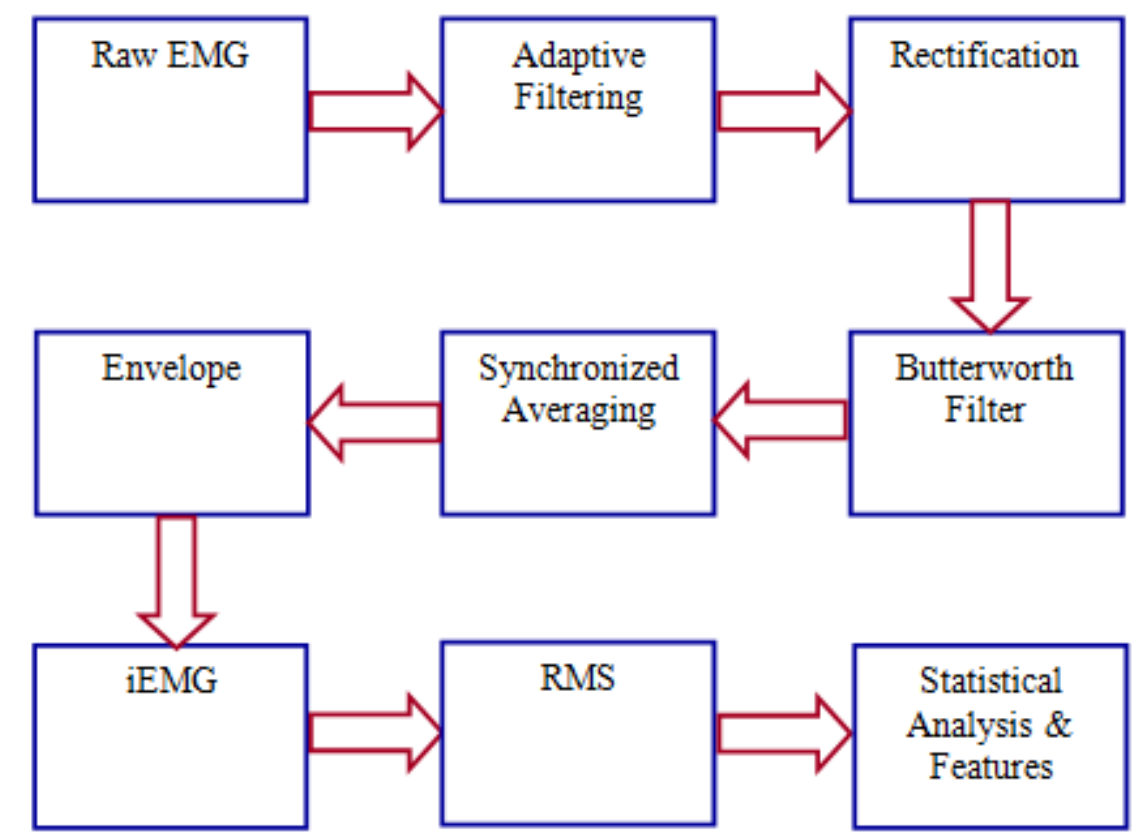

Figure 3.13: EMG Signal Processing Block Diagram

Rectification was performed by computing the absolute value of the entire signal [25]. This made analysis between the signals easier, as the envelope created later only had to deal with positive values. Butterworth low-pass filtering was necessary for basic noise removal and 
smoothing of the signal [25]. Typical cut-off frequencies for a low-pass Butterworth RMG filter ranges from $5 \mathrm{~Hz}$ to $15 \mathrm{~Hz}$ [24, 60]. Additional filtering can also be performed if required, depending on the quality of the signals acquired. Some of these filters may include, but are not limited to, high-pass, band-pass, and/or chebyshev. Different EMG measuring devices can produce signals of varying quality. Some devices come with many built in filters that clean the EMG signal. The built in bandpass filter in the BioRadio was used as an initial filter.

Synchronized averaging was used to reduce error by taking multiple recordings into account [24]. The final EMG signal would be an average of the multiple trials. The EMG envelope produced a single outline that illustrated the overall pattern of the given EMG signal. This envelop was easily be compared with other each other by amplitude, as well as the overall behaviour. Once the envelope was computed the RMS and iEMG values were computed for further comparison. The RMS is a single value determined by squaring the data points, taking the mean of all the values and finally taking the square root of this value [24]. This value represents the power of each muscle contraction. The iEMG was computed by taking the area under the EMG plot, in other words the integral. The iEMG was plotted by having cumulative values for the entire EMG graph. The cumulative value quantifies the amount of activity produced by each muscle.

Further analysis was performed using statistical analysis. The mean was computed for each signal to determine the average amplitude of each muscle, relative to the MVC. The variance and standard deviations helped compare fluctuation levels between the muscle contractions.

\subsubsection{Butterworth Filter}

Before any analysis was performed, all signals were filtered to reduce noise and artifacts. A 4th order low-pass Butterworth filter was used to adjust the frequency spectrum of the signals. The frequency spectrum represents the signal in frequency domain, rather than the time domain. The frequency domain can help analyse the signal in parts, and clearly portray the changes made to the signal via mathematical operations. The low-pass Butterworth filter helped create a ripple less frequency response and was designed using equation 3.3 [24]. 


$$
|H(\Omega)|^{2}=\frac{1}{1+\left(\frac{\Omega}{\Omega_{c}}\right)^{2 N}}=\frac{1}{1+\varepsilon^{2}\left(\left(\frac{\Omega}{\Omega_{p}}\right)^{2 N}\right.}
$$

In equation $3.3, \mathrm{~N}$ is the filter order, $1 /\left(1+\varepsilon^{2}\right)$ is the band edge value, $\Omega_{c}$ is the corner frequency, and $\Omega_{p}$ is the pass-band edge frequency.

\subsubsection{Synchronized Averaging}

Averaging multiple trials of the same signal can help reduce noise and improve signal-tonoise ratio (SNR). This is done by identifying the noiseless, repetitive signal. Synchronized averaging was performed using the following equation [24]:

$$
y_{k}(n)=x_{k}(n)+n_{k}(n)
$$

In equation $3.4, k$ represents the number of signal copies, $x_{k}(n)$ is the original signal, and $n_{k}(n)$ is the noise. Equation 3.4 can be extended to include multiple copies of the signal $(\mathrm{M})$, as seen in equation 3.5. As the number of copies of the signal increases, the amount of noise decreases [24].

$$
\sum_{k=1}^{M} y_{k}(n)=\sum_{k=1}^{M} x_{k}(n)+\sum_{k=1}^{M} n_{k}(n)
$$

This step not only helped reduce noise, but it also made up for slight misalignments and time differences during data acquisition.

\subsubsection{RMS and iEMG}

Once the filtering and averaging was complete, the envelope of the EMG was computed for analysis. The muscle activity and behaviour were compared using RMS and iEMG. Equation 3.6 was used to compute the RMS values.

$$
x_{r m s}=\sqrt{\frac{1}{n}\left(x_{1}^{2}+x_{2}^{2}+x_{3}^{2}+\cdots+x_{n}^{2}\right)}
$$

In this equation, $x$ represents the signal and $n$ represents the number of samples. Areas under the envelopes of the EMG were computed by integrating the envelops, using the following equation: 


$$
i E M G=\int_{0}^{t} E M G_{\text {envelope }} d t
$$

\subsubsection{Motion Data Processing}

Motion data acquired from the three spatial directions ( $\mathrm{x}, \mathrm{y}$, and $\mathrm{z}$ ) were combined using equation 3.8 .

$$
V M U=\sqrt{\left(x^{2}+y^{2}+z^{2}\right)}
$$

A moving average filter was used to remove noise, while computing the average motion between the subjects. Equation 3.9 represents the moving average filter used on the motion data [24].

$$
y(n)=\frac{1}{M} \sum_{k=0}^{M-1} x[n-k]
$$

The linear acceleration and angular velocity data acquired from the neck and body were normalized and compared by taking the differential between neck and body motion. A window size (M) of 0.2 seconds was used.

\subsubsection{Statistical Analysis}

The EMG signals were further compared using the following parameters: (1) mean, (2) standard deviation, and (3) variance. The normal distribution pattern was used to analyze how the EMG signals were distributed, relative to the mean, under the four conditions mentioned above. The probability density function for a normal distribution can be depicted using the following equation[61]:

$$
f(x, \mu, \sigma)=\frac{1}{\sigma \sqrt{2 \pi}} e^{-\frac{1}{2}\left(\frac{x-\mu}{\sigma}\right)^{2}}
$$


The symbols $\mu$, and $\sigma$ represent mean and standard deviation, respectively. The mean and standard deviations were computed for signals acquired from each muscle, under the four conditions, using equations 3.11 and 3.12 .

$$
\mu=\frac{\sum x}{n}
$$

In equation $3.11, n$ is the number of samples, and $x$ represents the data value.

The standard deviations and variances helped determine how much the signals differed from the mean, as well as the fluctuation levels of the signals acquired. The equation used can be found below.

$$
\sigma=\sqrt{\frac{\sum_{i=1}^{n}\left(x_{i}-\bar{x}\right)^{2}}{n-1}}
$$

In equation 3.12, $\sigma$ is the standard deviation $x_{i}$ is the data value, and $\bar{x}$ is the mean of $x_{i}$.

$$
\sigma^{2}=\frac{\sum\left(x_{i}-\bar{x}\right)^{2}}{n-1}
$$

In equation $3.13, \sigma^{2}$ represents the variance

\subsubsection{Features}

Further analysis was performed by comparing amplitudes, zero crossings (ZC), slope sign changes (SSC), and waveform lengths (WL). The amplitude provides information relating to the power of the muscle contractions. The number of zero crossings measures the amount of times the signal amplitude crosses zero. This can help provide information relating to the frequency of contractions and muscle fatigue. Zero crossings can be represented by equation 3.14 [62].

$$
\begin{gathered}
Z C=\sum_{n=1}^{N-1}\left[\operatorname{sgn}\left(x_{n} \times x_{n+1}\right) \cap\left|x_{n}-x_{n+1}\right| \geq \text { threshold }\right] \\
\operatorname{sgn}=\left\{\begin{array}{c}
1, \text { if } x \geq \text { threshold } \\
0, \text { otherwise }
\end{array}\right.
\end{gathered}
$$


The SSC (represented in equation 3.15) indicates the number of times the signal changes slope. SSC can provide information relating to the stages of muscle contractions (activation, contraction, recharging, and relaxation) [63].

$$
\begin{gathered}
S S C=\sum_{n=2}^{N}\left[f\left(x_{n}-x_{n-1}\right) \times\left(x_{n}-x_{n+1}\right)\right] \\
f(x)=\left\{\begin{array}{c}
1, \text { if } x \geq \text { threshold } \\
0, \text { otherwise }
\end{array}\right.
\end{gathered}
$$

Finally, the waveform length provides information relating to the length of muscle activity. The equation for waveform length can be seen in equation 3.16 [63].

$$
W L=\sum_{n=1}^{N}\left|x_{n+1}-x_{n}\right|
$$

In equations $3.14,3.15$, and $3.16, x$ represents the $\mathrm{EMG}$ data point and $\mathrm{N}$ represents the signal length.

\subsection{Chapter 3 Summary}

This chapter has discussed the devices, preparations, and processing techniques used in this study. The MaxFlight motion simulator provided a fully enclosed environment to test within. The BioRadio, with its multifunctional capabilities, helped measure EMG, ECG, linear acceleration, and angular velocity data, simultaneously. Furthermore, ECG crosstalk can occur in EMG signals acquired from areas with stronger pulse (closer to the heart). The adaptive filtering technique was used to successfully remove ECG crosstalk from EMG and the additional processing techniques were used create smooth comparable signals. Finally, statistical analysis along with features such as ZC, SCC, and WL were used to provide accurate information relating to EMG signal patterns. 


\section{Chapter 4}

\section{Results and Discussion}

$\mathrm{T}$

The following section discusses the results obtained from 20 subjects, 6 muscles, in the backward and forward pitch plane, with and without visuals. MATLAB was used to process the data and create results and plots. The EMG signals between the different muscles varied in signal strength and power. However, the overall pattern of the EMG signals were similar. For that reason, additional graphs focused on the right sternocleidomastoid muscle, when comparing the EMG, acceleration, and velocity profiles. This section focuses on the average results obtained from all the subjects. Extensive data tables can be found in Appendix A. The first half of this section focuses on the EMG signals acquired in the backward and forward pitch plane, with and without visuals. The second half of this section focuses on the linear acceleration and angular velocity profiles, under the same conditions.

\subsection{Graphical Results of Neck Muscle Activity}

\subsubsection{Adaptive Filtering Results}

Before the EMG results were process, ECG crosstalk from the neck was removed using adaptive filtering. Figure 4.1, shows an example output from the filter.

\subsubsection{Backward Rotation}

The normalized average muscle activity for each muscle, during backward rotation without VR, can be seen in Figure 4.2. Figure 4.3 illustrates the average data acquired while VR 

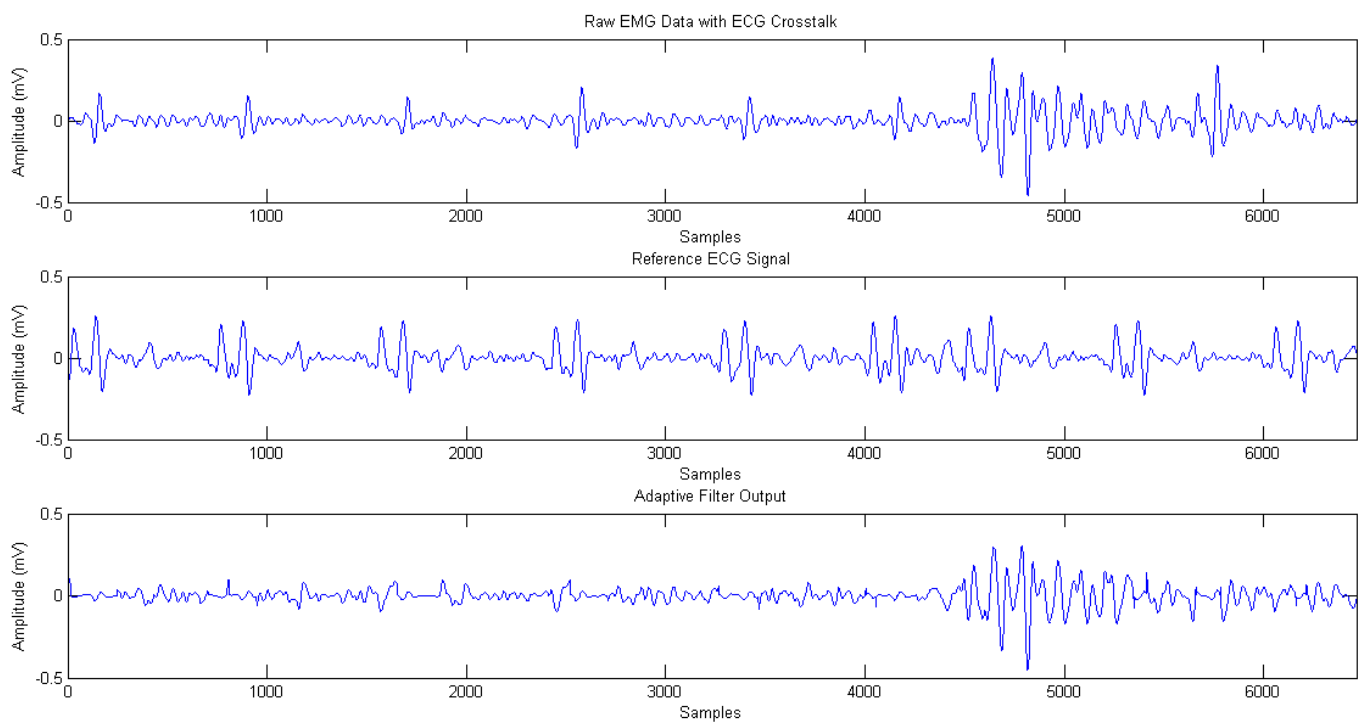

Figure 4.1: ECG Crosstalk Removal 
Average EMG with Envelope and RMS
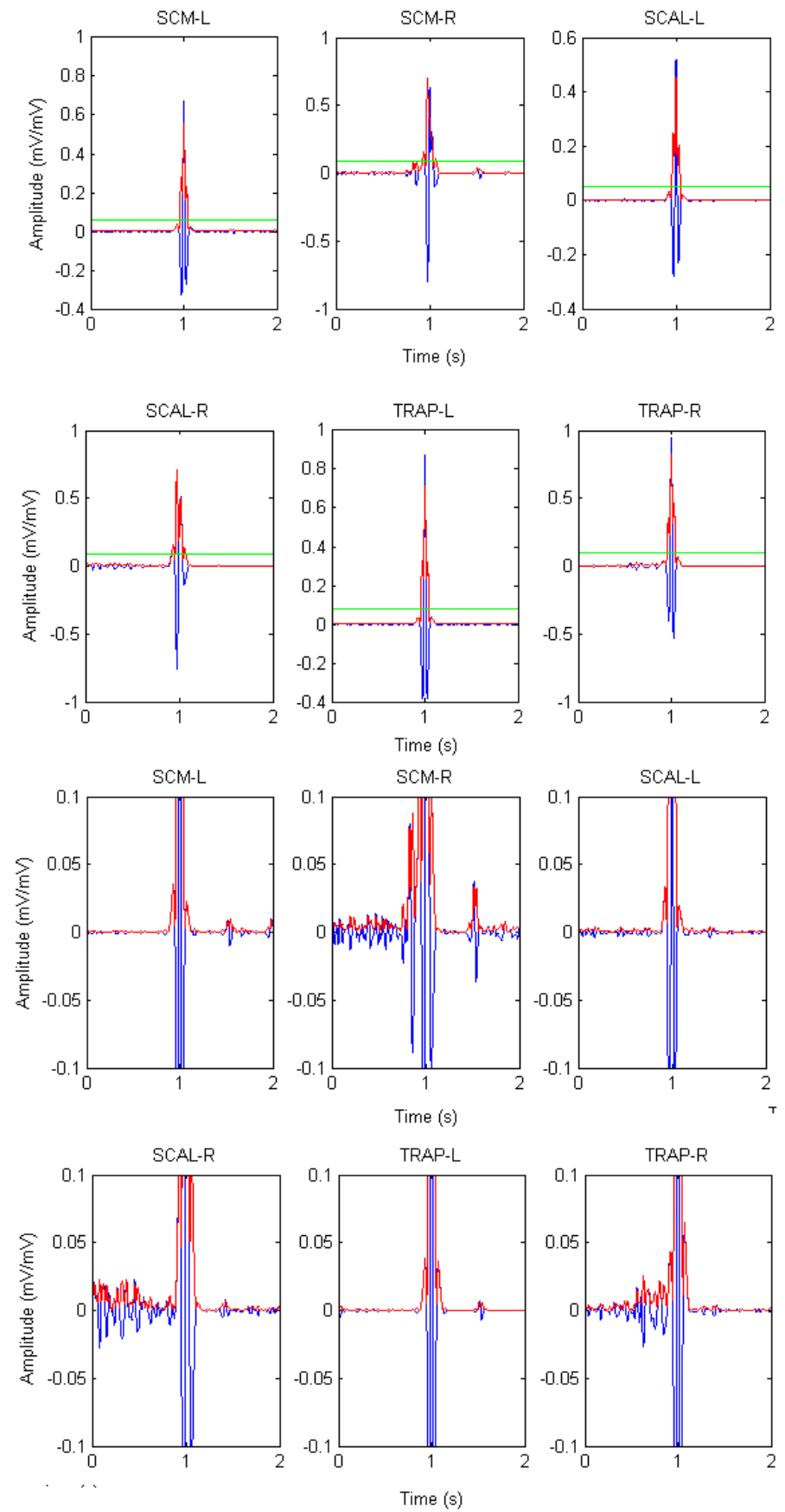

Figure 4.2: EMG for Backward Rotation with no Visuals (red = envelope; blue = EMG) 
was present. In these figures, the blue line represents the raw EMG, the red line represents the envelope of the filtered and rectified EMG, and the green line shows the RMS value, for each envelope. The first 2 rows of plots show the entire signal, while the last 2 rows shows a close-up view by scaling the y-axis. The close-up view shows a better view of the activity during motion, as the neck muscle contraction are very small during this time. The highest peaks can be observed toward the end of motion, at around the 1 second mark. This point in time is also when the subject experiences the final jerky motion, resulting from a sudden stop. The right muscles show higher activity before the final jerk. This represents the muscle activity that occurred throughout motion. However, the activity during motion is a lot smaller in amplitude, compared to the final jerk. Since the movement only lasted about second, this may not have been enough time for the muscles to adapt to the motion until the very end. Testing the neck muscle reactions for longer rides may show how the muscle adapt to the changes, once they are expected.

When looking at the amplitudes, the TRAP muscle showed slightly higher peaks of about $0.9 \mathrm{mV}$. The other muscles were closer to about $0.5 \mathrm{mV}$. This differentiates between the front and back muscles. The TRAP muscles, located in the back, are much larger and spread across a much wider area, compared to the SCM and SCAL muscles in the front.

In Figure 4.3, the peaks are evident during jerk. However, when looking at the close-up plots in this figure, there is slightly more muscle activity taking place during movement by the TRAP muscles. A few additional noticeable peaks are present for the left TRAP muscle. This muscle shows a stronger initial contraction right after the start of motion. There were not any mentionable differences between the amplitudes when measuring with VR, as opposed to without. In this case, the strength of contractions maintains the same, where the TRAP muscles had stronger contractions during jerk ranging from 1 to $1.5 \mathrm{mV}$. Here again, the SCAL muscles had the lowest amplitudes around 0.3 to $0.4 \mathrm{mV}$, which can be justified by their thinner size compared to the SCM and TRAP muscles.

One interesting factor was the slightly higher amplitudes by the front left muscles, while visuals were present. This shows a slight rotation of head toward the opposite side of contraction (right side). The subjects showed a slight rotation to the right. This can be explained by the positioning of the subjects, during the experiments. Since the motion simulator had two seats, all 
Average EMG with Envelope and RMS
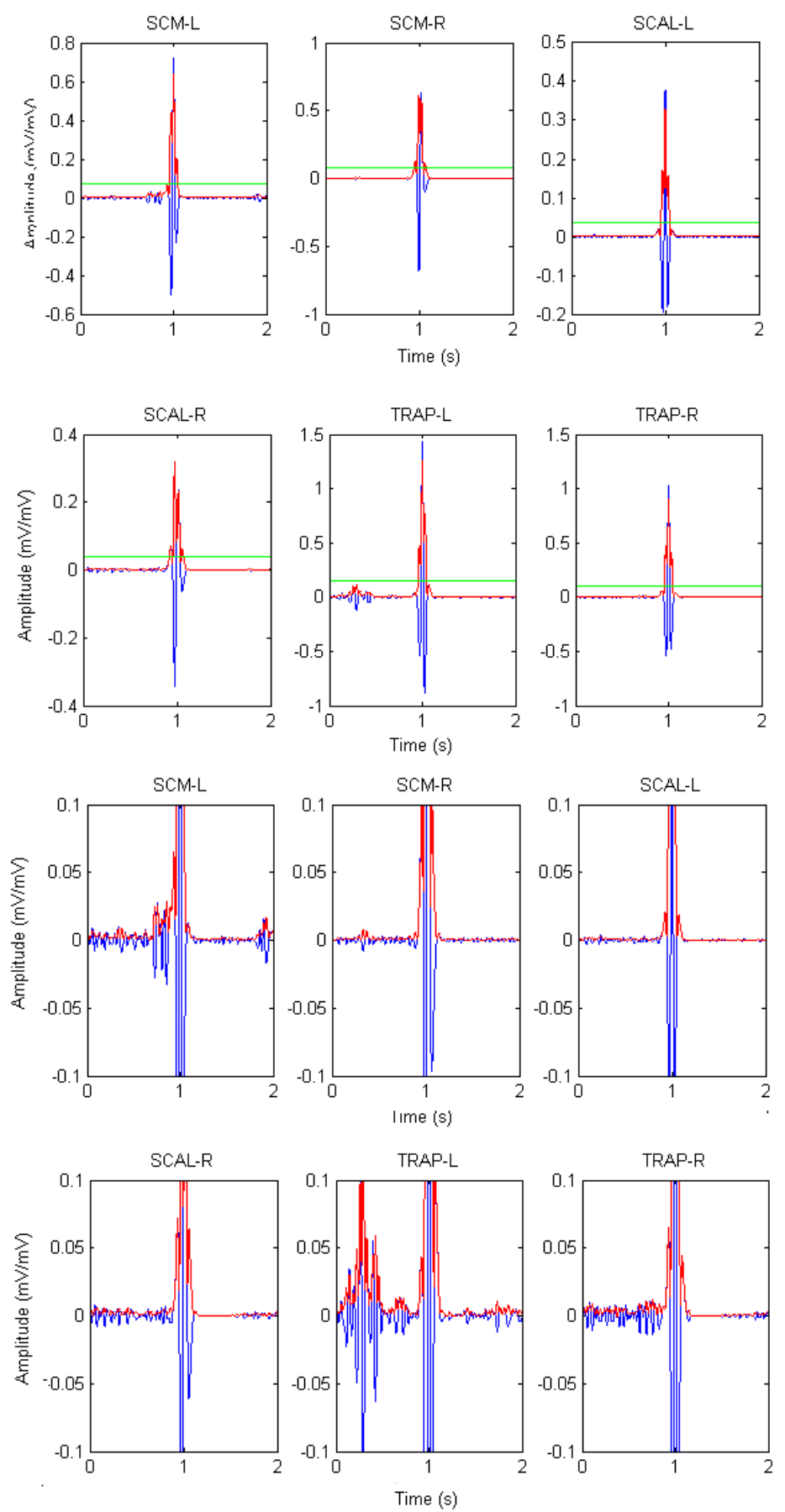

Figure 4.3: EMG for Backward Rotation with Visuals (red = envelope; blue = EMG) 
subjects were seated on the left seat, to maintain consistency. Hence, the subjects lacked a central view of the screen.

All subjects were positioned slightly left to the centre of the screen. This can explain why there were slightly stronger signals from the left muscles, as the head was slightly turned toward the centre of the screen. This was noticeable while the VR was present. When there were no visuals present, the amplitudes between the left and right muscles were closer together. It could be assumed that the subjects were not focusing on the screen during these trials, and had their heads facing straight forward.

When looking at the iEMG graphs for the backward motion. It is evident that area under the curve increases dramatically towards end of motion. In Figures 4.4 and 4.5, there is not much difference between the amount of work performed by the front muscles. The back muscles, however, showed greater area under the curve when VR was present. This can indicate two things, greater amplitudes or more activity resulting in frequent oscillations, when VR was present, as they result in larger integrals. The front neck muscles showed a decrease in work while VR was present. These lower contraction levels and activity levels could also be due to the headrest being present during motion. The head and neck had support during the backward rotations.

\subsubsection{Forward Rotation}

Figures 4.6 and 4.7 show the EMG activity for each muscle, during forward rotation, with and without VR. From first glance, it is obvious that there is greater muscle activity during forward rotation. The peaks during jerk are higher than the peaks in the backward motion, indicating stronger contractions. A similar pattern observed, is back muscles (left and right TRAP) having higher peak amplitudes, however, during forward rotation we see the front muscles showing higher amplitudes as well. The SCM muscles are very close in amplitude to the TRAP muscles. The SCAL muscles' amplitudes have also increased from $0.3 \mathrm{mV}$ during backward rotation to about $0.6 \mathrm{mV}$ during forward rotation. This shows that the front muscles definitely produce stronger contractions in the forward motion. There are a lot more oscillations and the frequency of contractions are greater. This show that that neck muscles are not only 

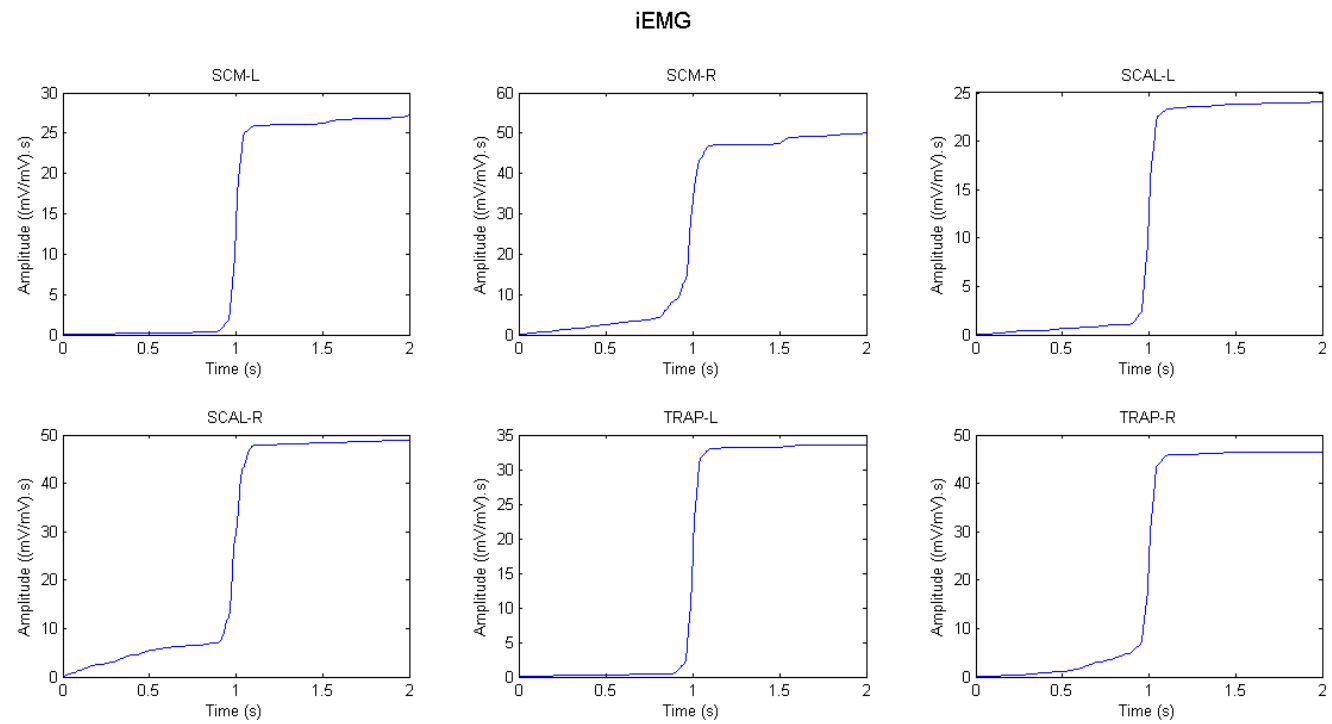

Figure 4.4: iEMG for Backward Rotation with no Visuals
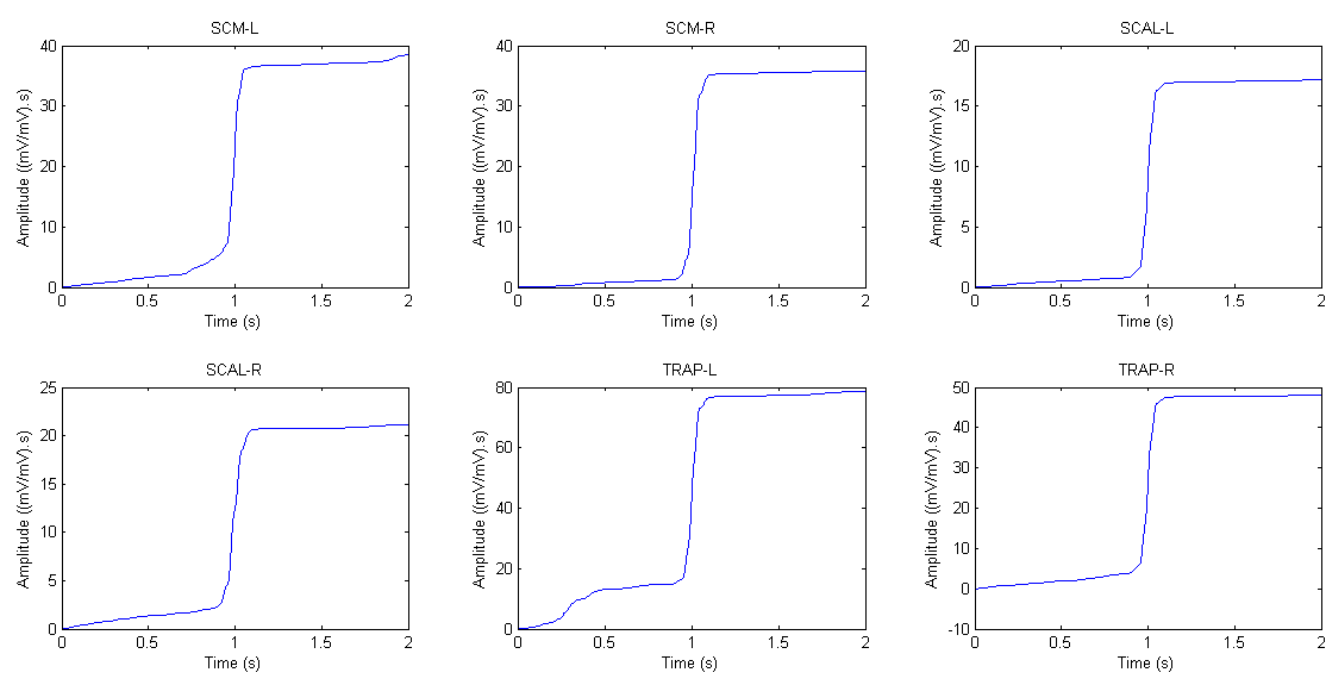

Figure 4.5: iEMG for Backward Rotation with Visuals 
Average EMG with Envelope and RMS
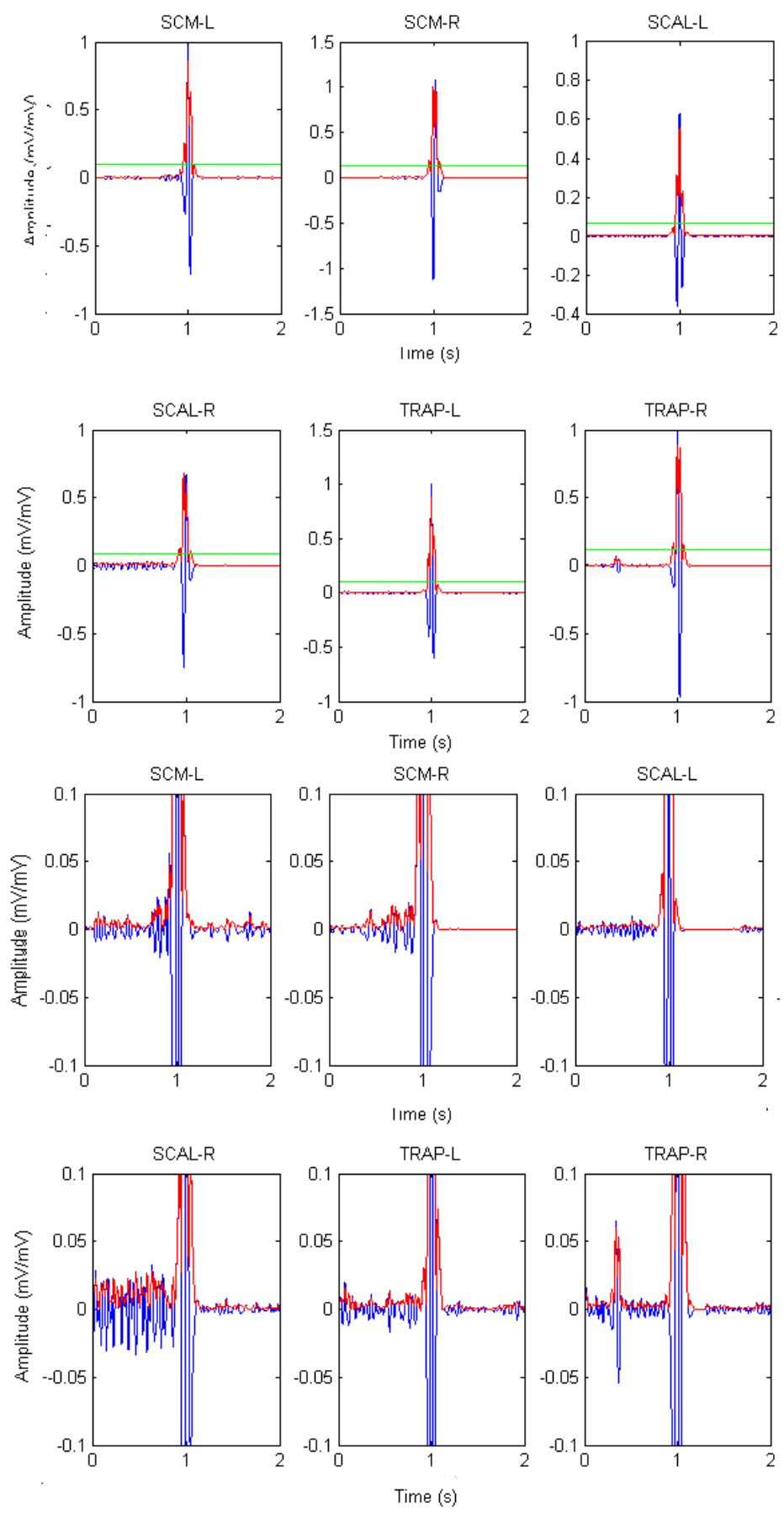

Figure 4.6: EMG for Forward Rotation with no Visuals (red = envelope; blue = EMG) 


\section{Average EMG with Envelope and RMS}
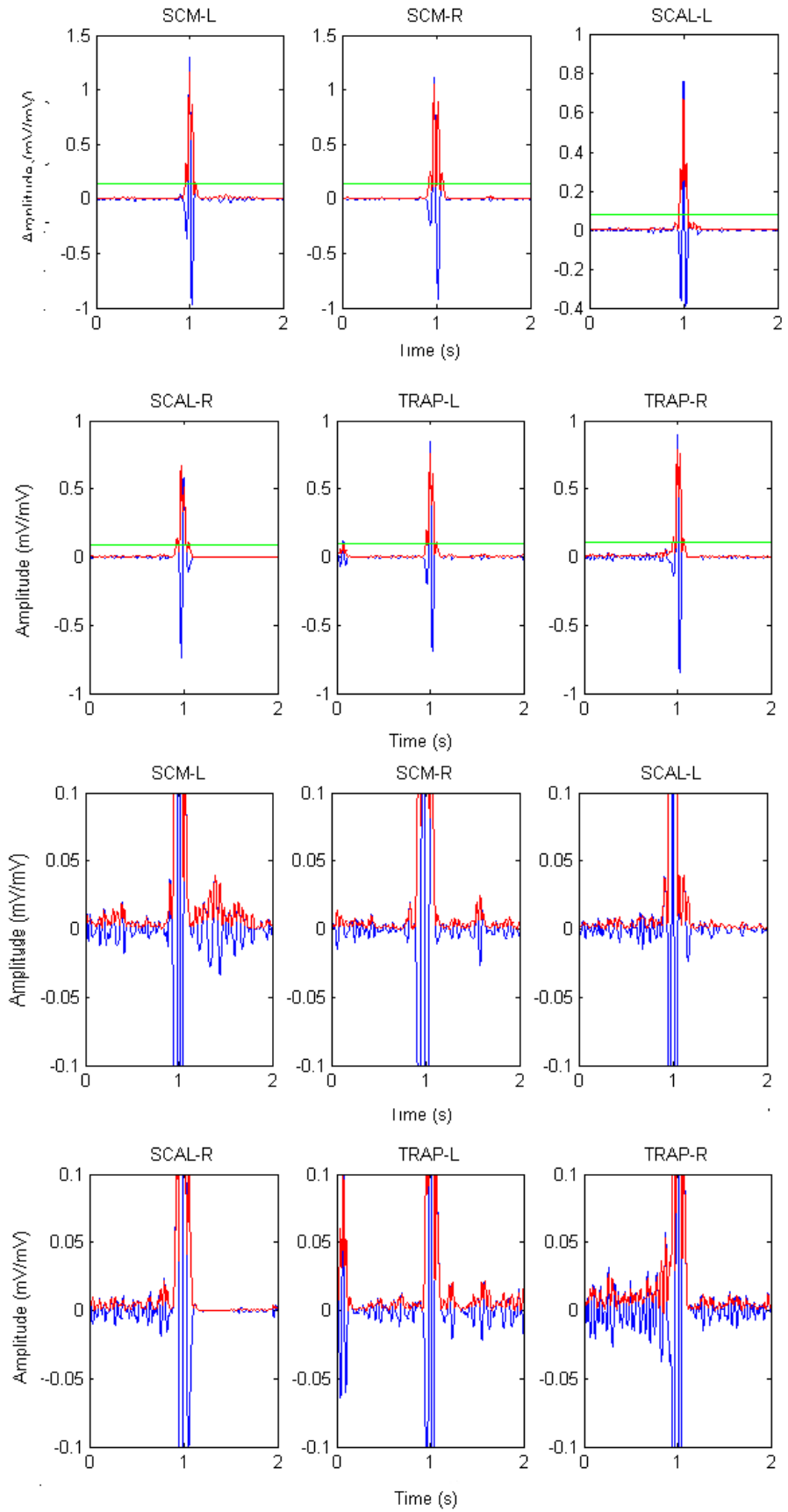

Figure 4.7: EMG for Forward Rotation with Visuals (red = envelope; blue = EMG) 
producing stronger contractions, but also a great number of contractions. On the other hand, the amplitudes between the right and left muscles were relatively the same.

When taking a look at the graphs for forward motion with visuals, all the muscles show an increase in activity, with higher frequent oscillations, and more contractions. So, the muscles are definitely more active with VR present, and during forward motions. Since the head has no support while rotated in the forward pitch plane, the muscles have to put in more work to stabilize the head, thus the higher amplitudes and increase in muscle activity. Here, as also seen in Figure 4.6, the SCM muscle amplitudes range from 1 to $1.5 \mathrm{mV}$. However, we do see the pattern of left muscles having slightly higher amplitudes, indicating a slight head rotation to the right. For example, the EMG of the left SCAL muscle reaches close to $0.8 \mathrm{mV}$ while the right SCAL muscle reached a maximum amplitude of about $0.6 \mathrm{mV}$. This is only evident in the front muscles, as they help rotate the head, while the TRAP muscles help with flexing and extending the neck. In this case, the left TRAP muscle shows the higher initial peak a few milliseconds after the start of motion.

Another interesting aspect that was not evident in the previous graphs, was the contractions after the jerk. One of the major impacts of VR was the increase in activity after the final jerk, during forward rotation. The left SCM muscles show the strongest example of postjerk contractions. This shows that when visuals were present, the neck muscles were active for a longer period of time. Not only are these contractions caused by the subjects focusing on something ahead, but having visuals with a focal point can help provide a sense of position that can be used to help stabilize the neck and head. These extra contractions in the end were missing during backward rotation, as the head was supported by the headrest after jerk. But, these postjerk contractions were also missing the forward motions without visuals present. This could also be a sign that the head and neck may not be reaching proper stability, when experiencing forward rotational motions in complete darkness.

Looking at Figure 4.8, it is evident that there is greater area under the curves for the signals acquired in forward motion. The anterior muscles also show a slightly steeper slope before the jerk at 1 second. This indicates greater muscle activity as the ride approached jerk. The SCM muscles had total iEMG ranging from 60 to $70(\mathrm{mV} / \mathrm{mV}) \cdot \mathrm{s}$, while they mostly ranged 
around $40(\mathrm{mV} / \mathrm{mV}) \cdot \mathrm{s}$ during backward rotation. The $(\mathrm{mV} / \mathrm{mV})$ unit is achieved after normalization, where the EMG signal is divided by the MVC. The unit for time becomes incorporated when computing the area under the curve, resulting in $(\mathrm{mV} / \mathrm{mV}) \cdot \mathrm{s}$. Furthermore, the right TRAP muscle showed a strong peak right after the start of motion.

The greater post-jerk activity can also be seen in Figure 4.9, as the slopes after the 1 second mark are steeper than the iEMG graphs seen in Figures 4.4, 4.5, and 4.8. The SCM muscles had the highest total area under the EMG signals. This follows the earlier trend of the SCM muscles being the most active during forward motions.

In Figures 4.10-4.13, the right SCM muscle EMG from all subjects are plotted, for each condition. Each colour represents the average between 3 trials for each subject. The largest peaks occurred from 0.8 to 1.2 seconds in all the graphs. However, the peaks slightly varied in time depends on the muscle reaction times of the subjects. Most of the EMG reached around 0.6 $\mathrm{mV}$ with a few signals that reach up to $0.8 \mathrm{mV}$. The amplitudes for rotation in the forward pitch plane range around 0.9 to $1.2 \mathrm{mV}$. The most important pattern to observe is that all the subjects produced the largest EMG peak close to the end of the ride, at the final jerk. Hence, the stop in motion seems to play a greater role than the start.

\subsection{Graphical Results of Head and Body Motion}

\subsubsection{Backward Rotation}

The following section looks at the accelerometer and gyroscope data acquired during motion, under the same conditions. One motion sensor was positioned behind the head where the parietal and occipital bones meet. The second sensor was placed on the torso near the upper rectus abdominis muscle. Motion data was acquired simultaneously with the EMG and ECG data. The common simulator motion in the neck and body motion data was accounted for by taking the differential between the neck and body motion. Additional graphs showing computed acceleration and jerk can be found in Appendix A. 

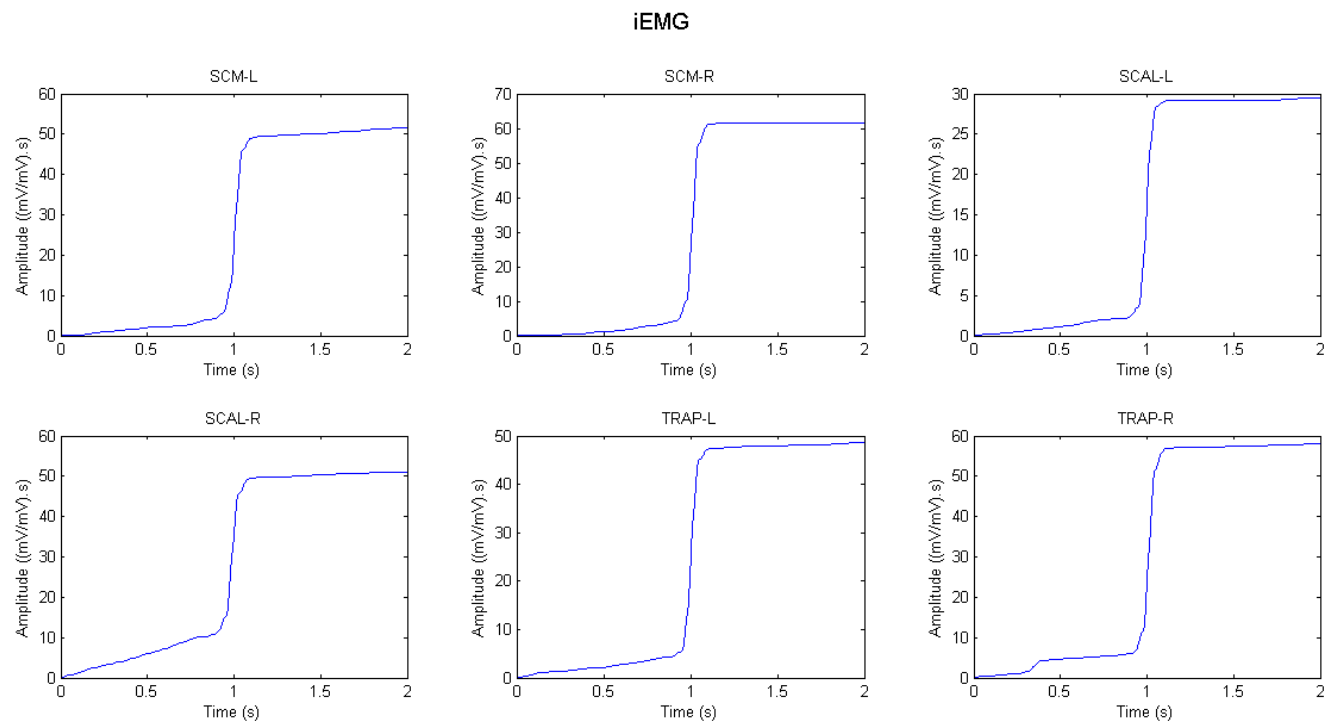

Figure 4.8: iEMG for Forward Rotation with no Visuals
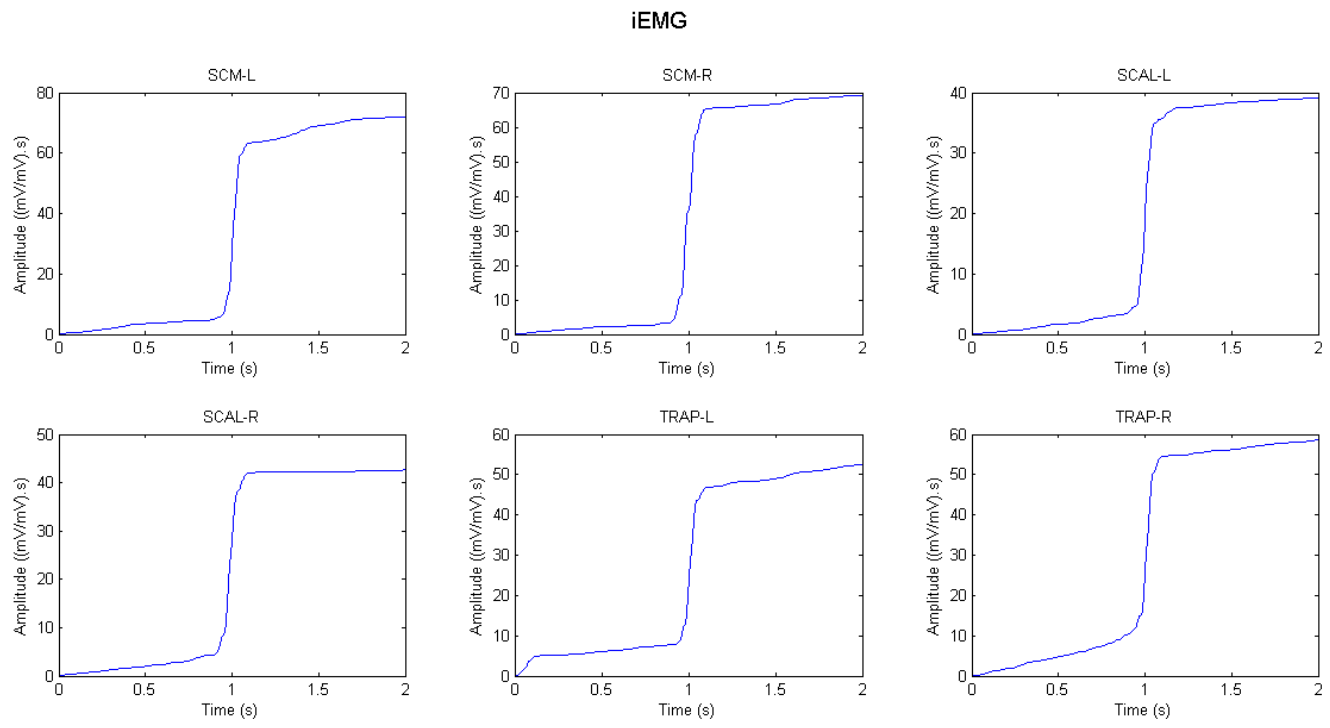

Figure 4.9: iEMG for Forward Rotation with Visuals 


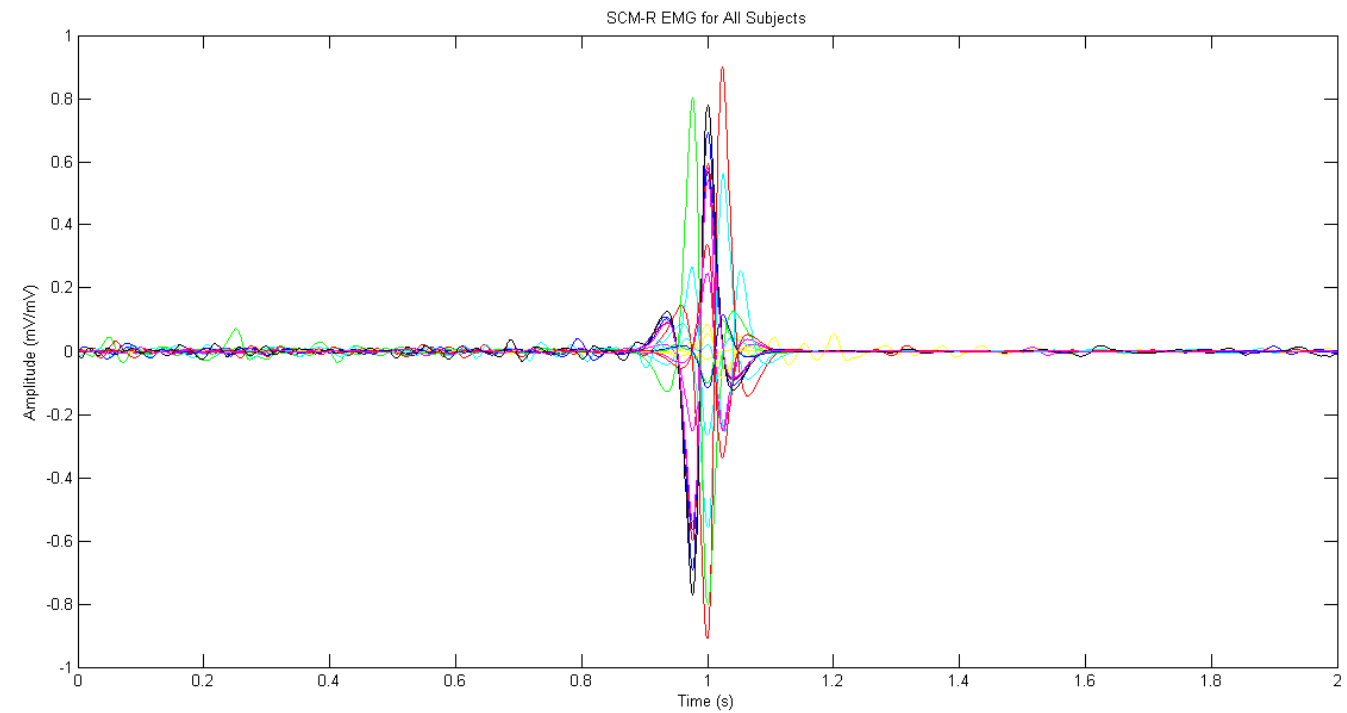

Figure 4.10: All EMG for Backward Rotation with no Visuals

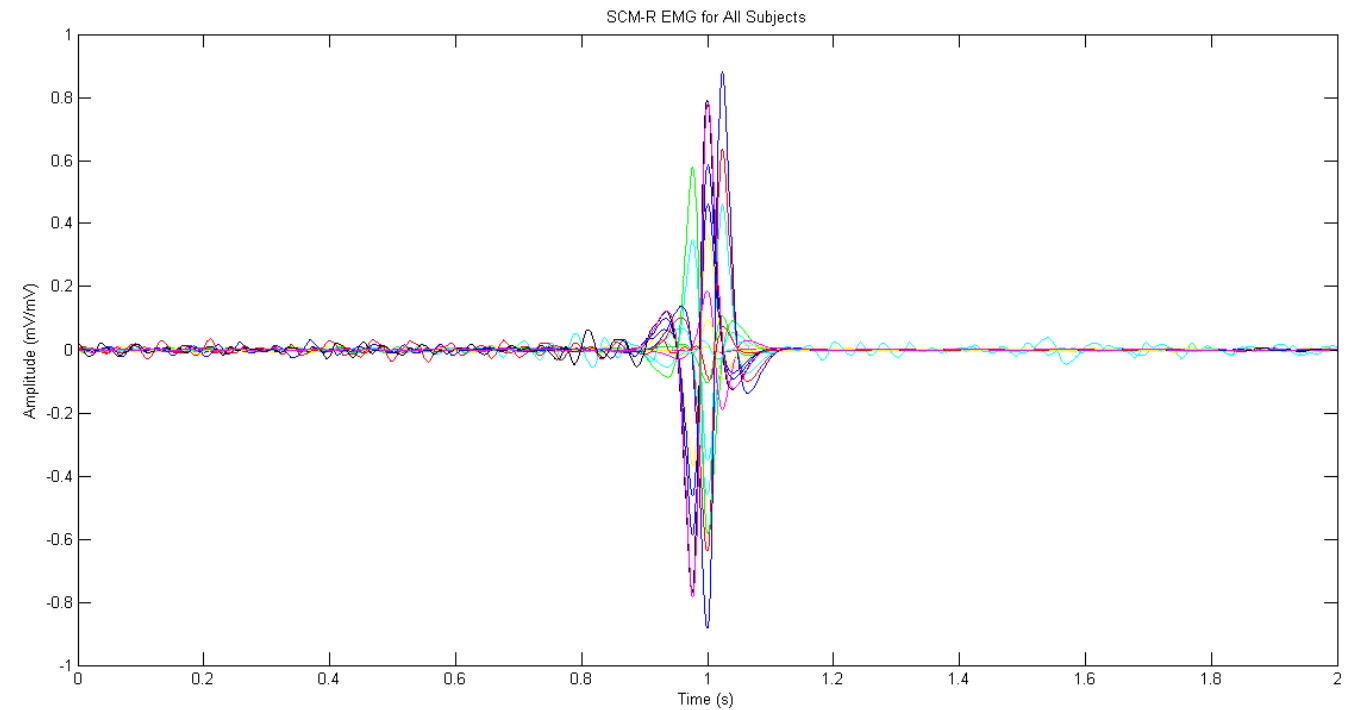

Figure 4.11: All EMG for Backward Rotation with Visuals 


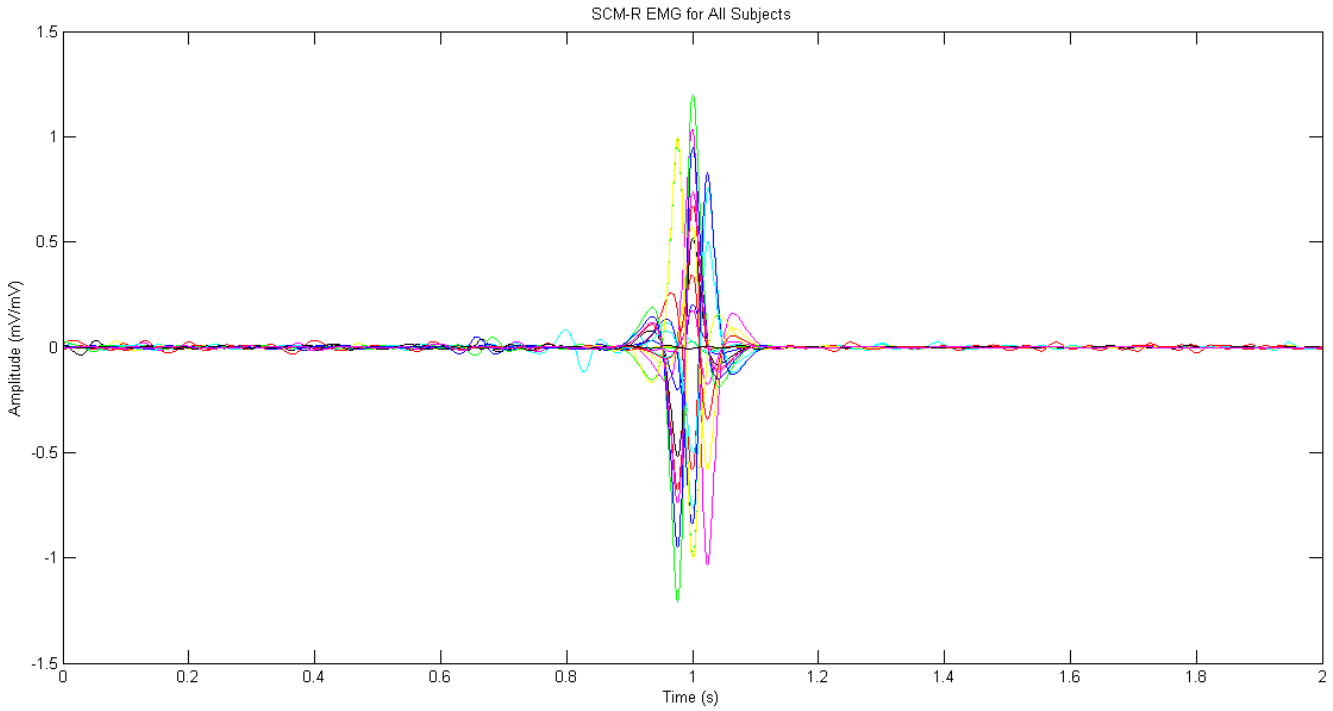

Figure 4.12: All EMG for Forward Rotation with no Visuals

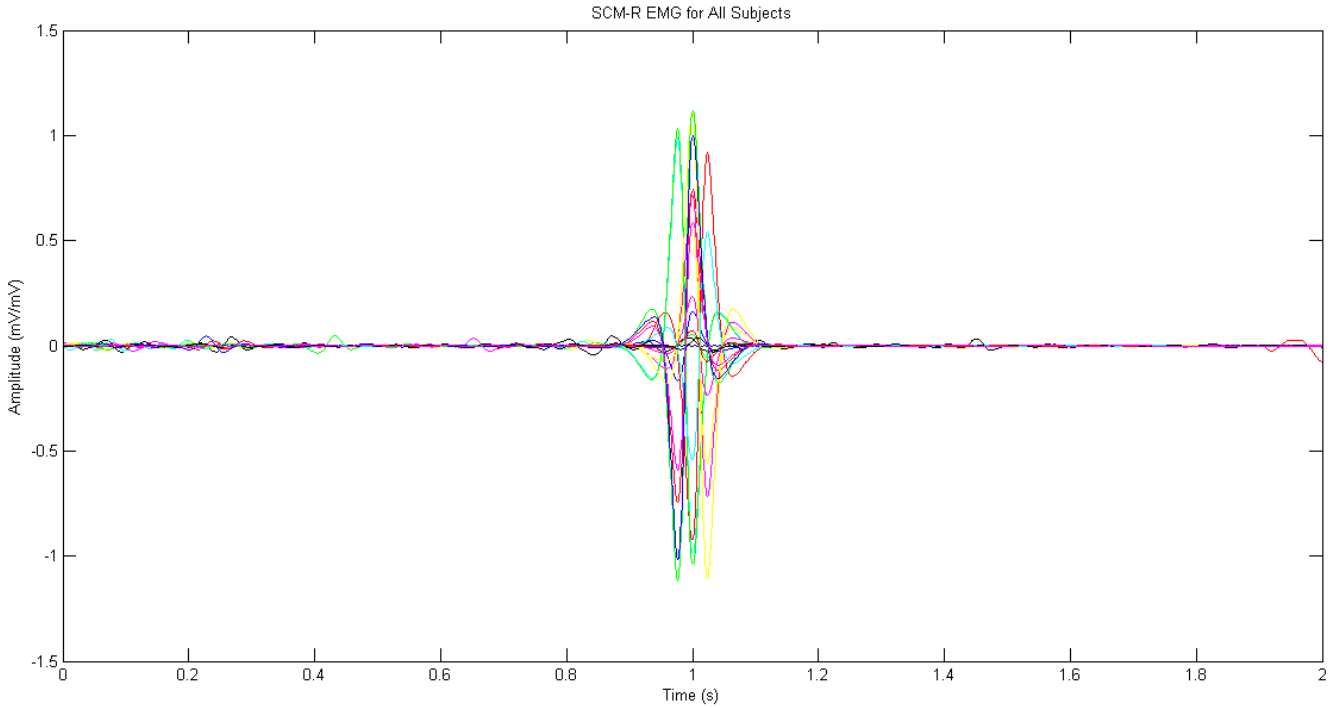

Figure 4.13: All EMG for Forward Rotation with Visuals 
Figure 4.14 shows the motion profile of the neck during backward rotation, without VR present. The plot to the left shows the linear acceleration measured using the tri-accelerometer, and the plot to the right shows angular velocity measured using the tri-axial gyroscope. The blue line represents motion across the yaw plane, the red line represents motion across the pitch plane, and the green line represents motion across the roll plane. As seen in the plot, there was greater angular motion, rather than linear motion. This makes sense, as the subjects were rotated about a fixed point. The largest degree of motion in the angular velocity graph can be seen across the $y-$ axis, as the subjects were rotated in the pitch direction. Since, all the subjects showed similar motion patterns, with slightly varying reaction times, the single motion profiles of the neck and body presented in this section were from random subjects. These graphs show the detail of motion and stabilization that become dampened in the averages. The following graphs show the differential motion averages of all subjects.

When looking at the motion profile of the neck, the linear acceleration graph shows an acceleration of the neck in the positive roll axis after the end of motion. This shows that the stabilization is achieved after the neck moves back with the body around jerk. In the angular velocity graph, it is interesting to see how the head moves during motion. The red line (pitch motion) shows initial motion of the head in the forward pitch plane, right after the start of motion. This movement is then followed by a larger degree of motion in the backward pitch plane. The maximum angular velocity occurs at the 1 second mark (the end of motion). During the time period of 1.2 to 2 seconds, the graph shows some post-motion stabilization. There are a few changes in slope signs that show posture adjustments. Moreover, the angular motion across the roll plane is very small, however there were some stabilization seen across the yaw plane.

Figure 4.15 shows the motion profile of the body during backward rotation, without VR present. Here, the linear acceleration mostly occurred during motion ( 0 to 1 seconds). When looking at the angular velocity graph, there is no initial jerk in the forward pitch plane, as noticed in the neck motion profile. The body begins moving in the direction of motion with the greatest velocity around the 1 second mark. One difference seen in Figure 4.14, compared to Figure 4.15, was the maximum angular velocity reached by the neck (about 90 degrees per second), 

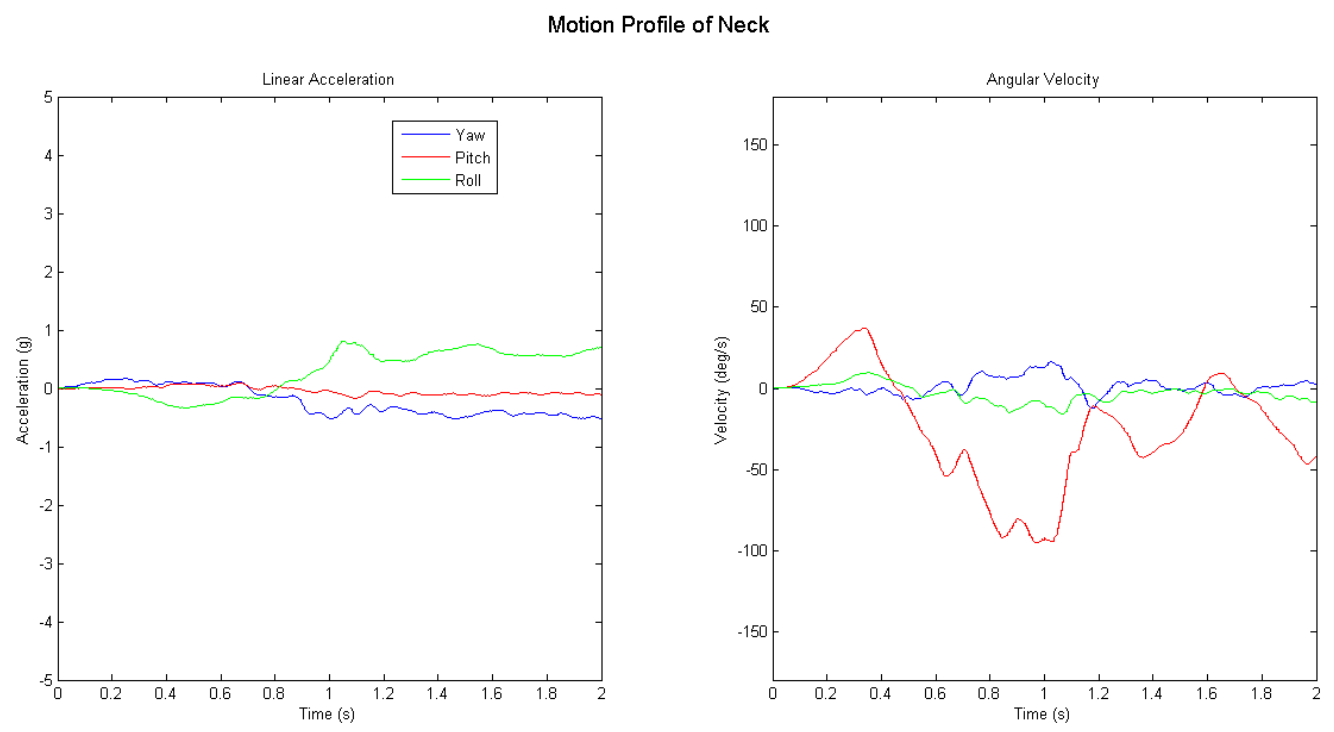

Figure 4.14: Motion Profile of Neck for Backward Rotation with no Visuals (red = pitch; blue = yaw; green $=$ roll)

versus the body (about 75 degrees per second). The angular profile of the body also shows more frequent slope sign changes throughout motion.

Figure 4.16 displays a comparison between the total motion produced by the neck and body, across all axes, in vector matrix units (VMU). Both the neck and body have greater motion towards the end of the ride, as the simulator comes to a sudden stop. The neck shows greater motion relative to the body. It is also evident, from Figure 4.16, that the body has a smoother profile, compared to the neck. The neck profile shows smaller motion pulses after the start of motion, as well as after the completion of motion. The green line in the neck profile graph shows the average EMG response by the right SCM muscle. The maximum contraction occurs during the highest range of motion from 0.8 to 1.2 seconds. This shows the neck muscles exerting the strongest contraction to prevent angular neck motion past a certain point during jerk. 
Motion Profile of Body
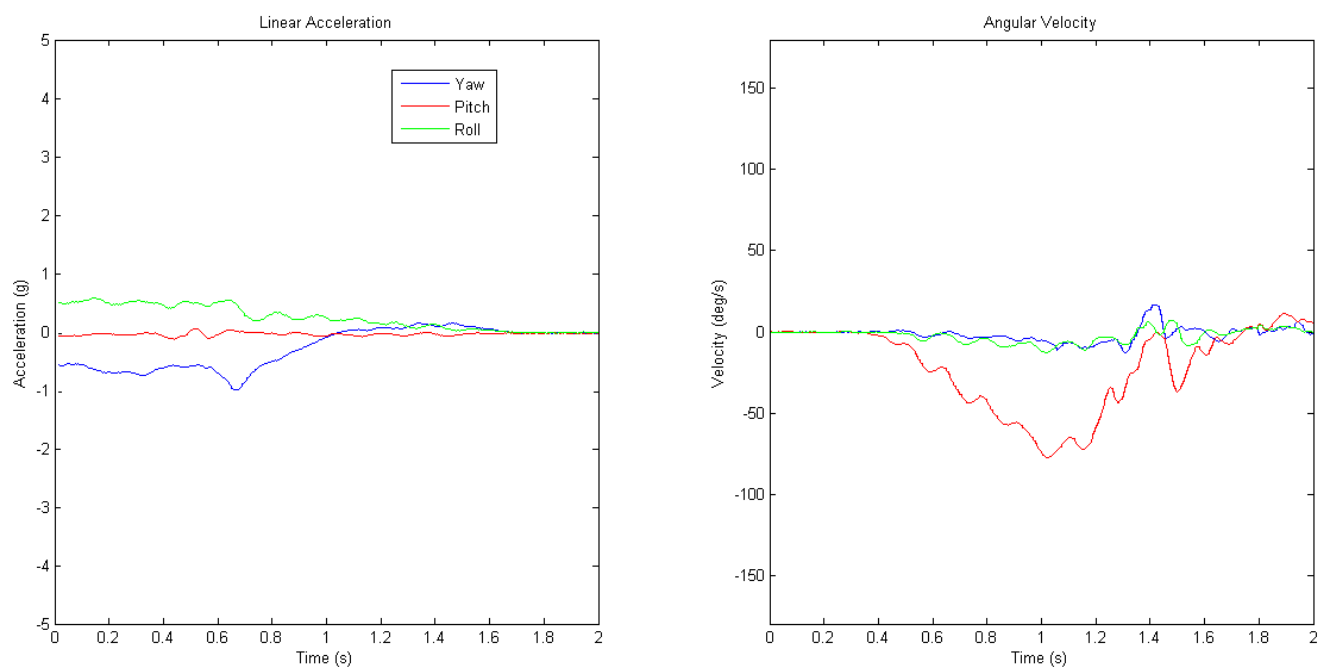

Figure 4.15: Motion Profile of Body for Backward Rotation with no Visuals (red = pitch; blue = yaw; green $=$ roll)
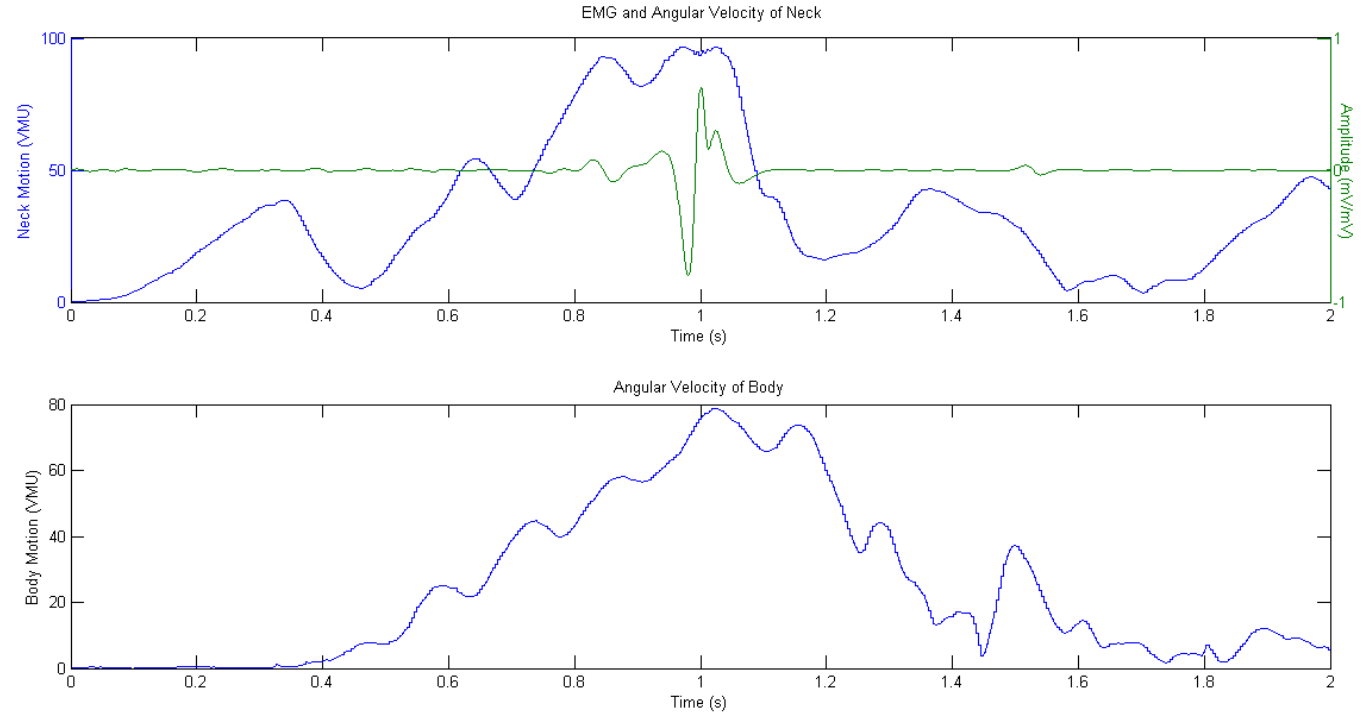

Figure 4.16: Comparison of EMG, Neck and Body Angular Velocity for Backward Rotation with no Visuals (blue = motion; green $=\mathrm{EMG}$ ) 
Further processing was performed to compute the differential motion between the neck and the head. This step normalizes the data and eliminates any common motion between the neck and body. Figure 4.17, shows all the computed differential motion profiles for each subject. The graph shows an overall similar pattern with slightly varying reaction times. All the differential motion profiles were averaged using a moving average filter with a window size of 0.2 seconds. Figure 4.18, shows the differential motion average of all subjects. The differential linear acceleration between the neck and body were very minimal. In the angular velocity graph, a positive VMU indicates dominance in neck motion, while a negative VMU indicates a dominance in body motion. In this graph, the neck had dominant motion most of the time. The profile begins with an initial peak of motion after the start of motion, after which the neck and body reached a common velocity (indicated by the zero differential at about 0.7 seconds). A greater peak of motion occurs at the stop of motion, around 1 second, and common velocity was reached again around 1.3 seconds. The profile ends off with some minor dominance in head motion that starts decreasing towards zero (stabilization).

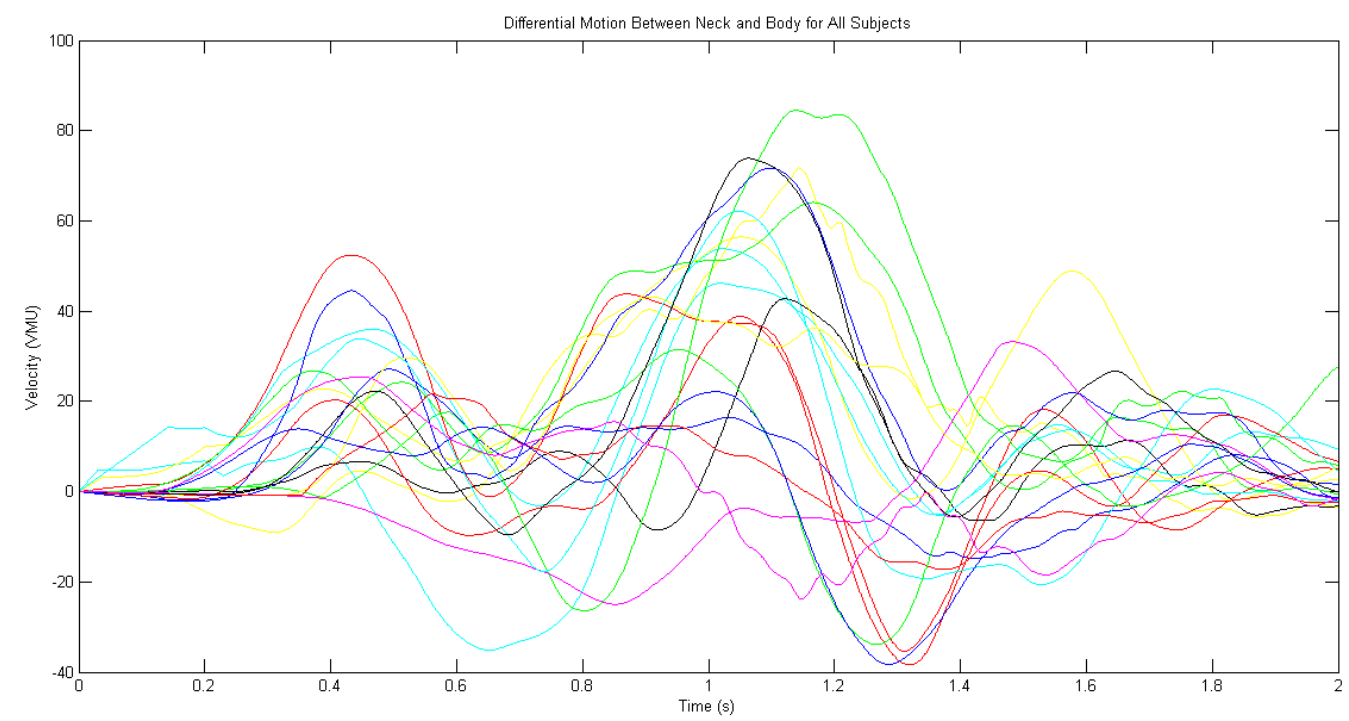

Figure 4.17: All Differential Motion Graphs for Backward Rotation with no Visuals 

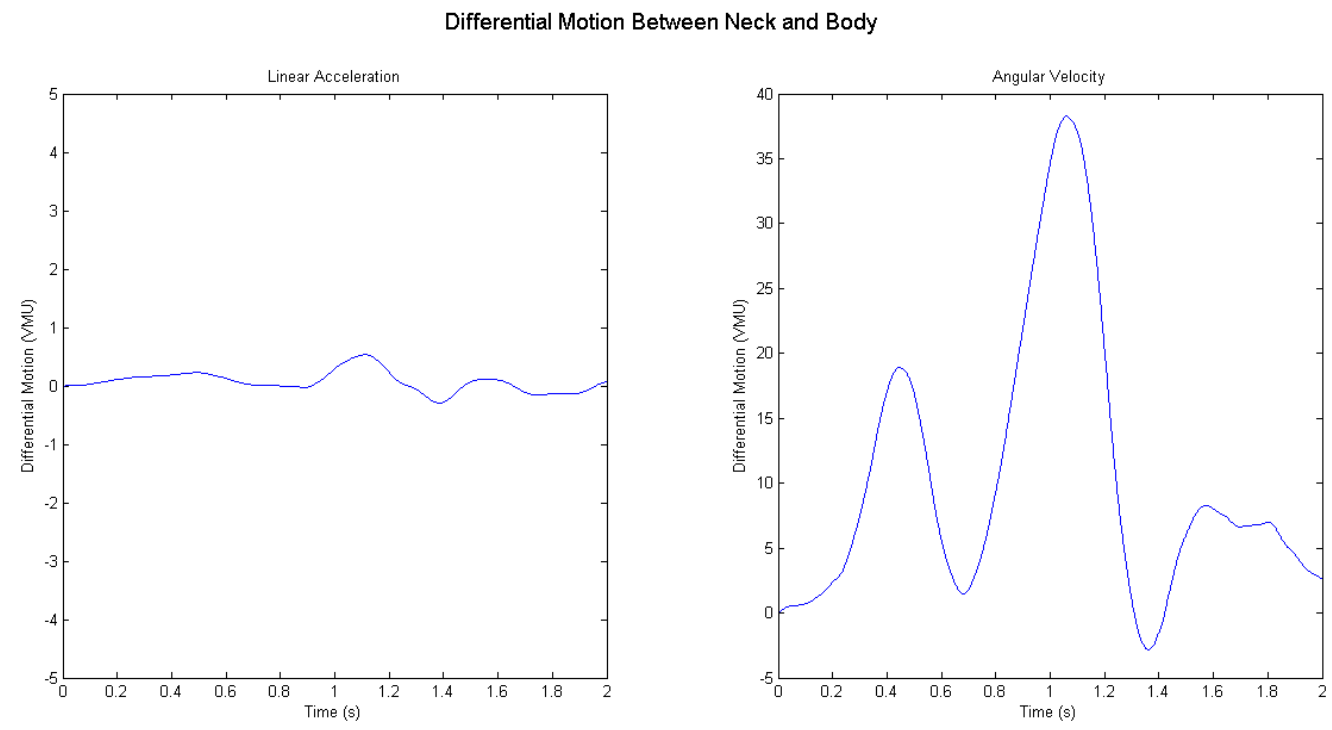

Figure 4.18: Average Differential Motion Graphs for Backward Rotation with no Visuals

Overall, for angular motions of 45 degrees per second, the peak angular velocity of the neck ranges from 35 to 40 degrees per second. The velocity in the backward motion is close to that of the simulator. This following part looks at the graphs acquired during backward motion with the presence of VR. As seen in Figure 4.14, the linear acceleration graph to the left of Figure 4.19 shows minimal motion of about $1 \mathrm{~g}$. There is forward acceleration, after motion has stopped, as well as a slight movement to the right (seen by the blue line), which may be due to the focus on the screen. The angular velocity graphs shows a similar overall pattern as seen before, without VR. The motion profile starts off with an initial jerk in the opposite direction of motion, followed by movement in the same direction as motion. This graph also shows a similar number of slope sign changes after the 1.2 second mark as Figure 4.15. One difference seen in this graph is that the motion in the pitch access is much smoother, with few oscillations and minor slope changes compared to Figure 15. 

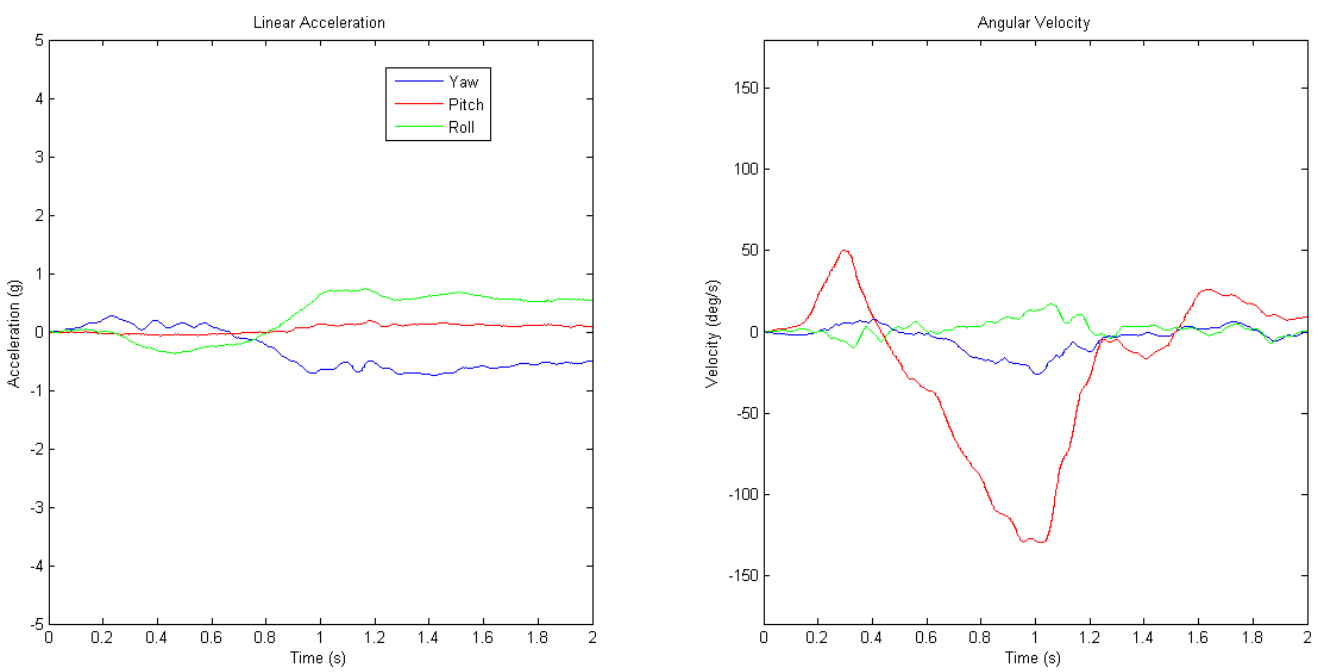

Figure 4.19: Motion Profile of Neck for Backward Rotation with Visuals (red = pitch; blue = yaw; green $=$ roll $)$

Figure 4.20 shows the motion profile of the body during backward motion, with VR present. One major difference in linear body motion is that the majority of the linear acceleration occurs during motion, rather than after. Here again, it is evident that the body does not have the initial jerk of motion in the opposite direction of motion as the neck does. One difference found in Figure 4.20, is the stronger oscillations compared to no visuals present. This graph shows more swaying of the body as the body works to stabilize itself. The presence of VR, definitely shows a difference in how the body moves throughout motion. The magnitudes of the signals are relatively the same for both conditions.

When looking at the sum of both neck and body movements in Figure 4.21, The neck reaches a slightly higher magnitude than the body. Although the body signal shows more prominent swaying, compared to when visuals were not present, the neck motion shows a much smoother change in angular velocity. The presence of VR, seems to be impacting how sudden the neck changes positions. 


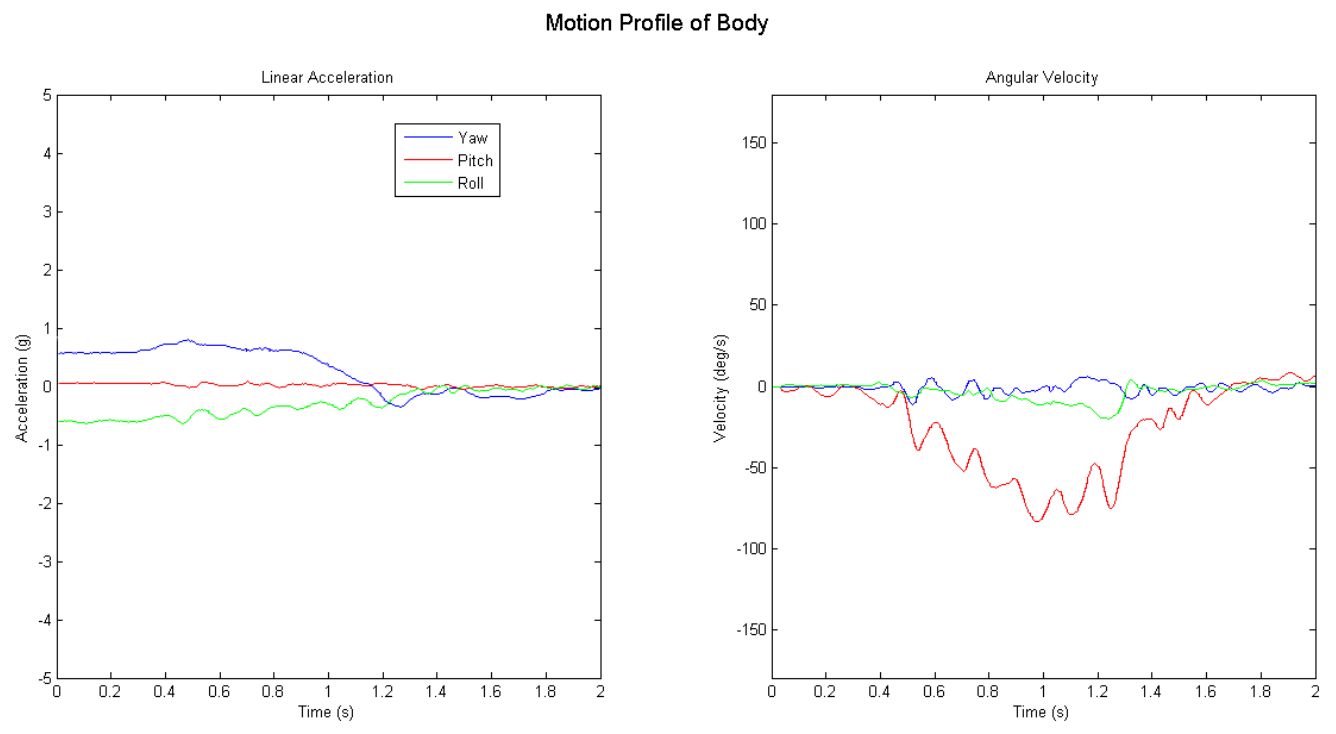

Figure 4.20: Motion Profile of Body for Backward Rotation with Visuals (red = pitch; blue = yaw; green $=$ roll)
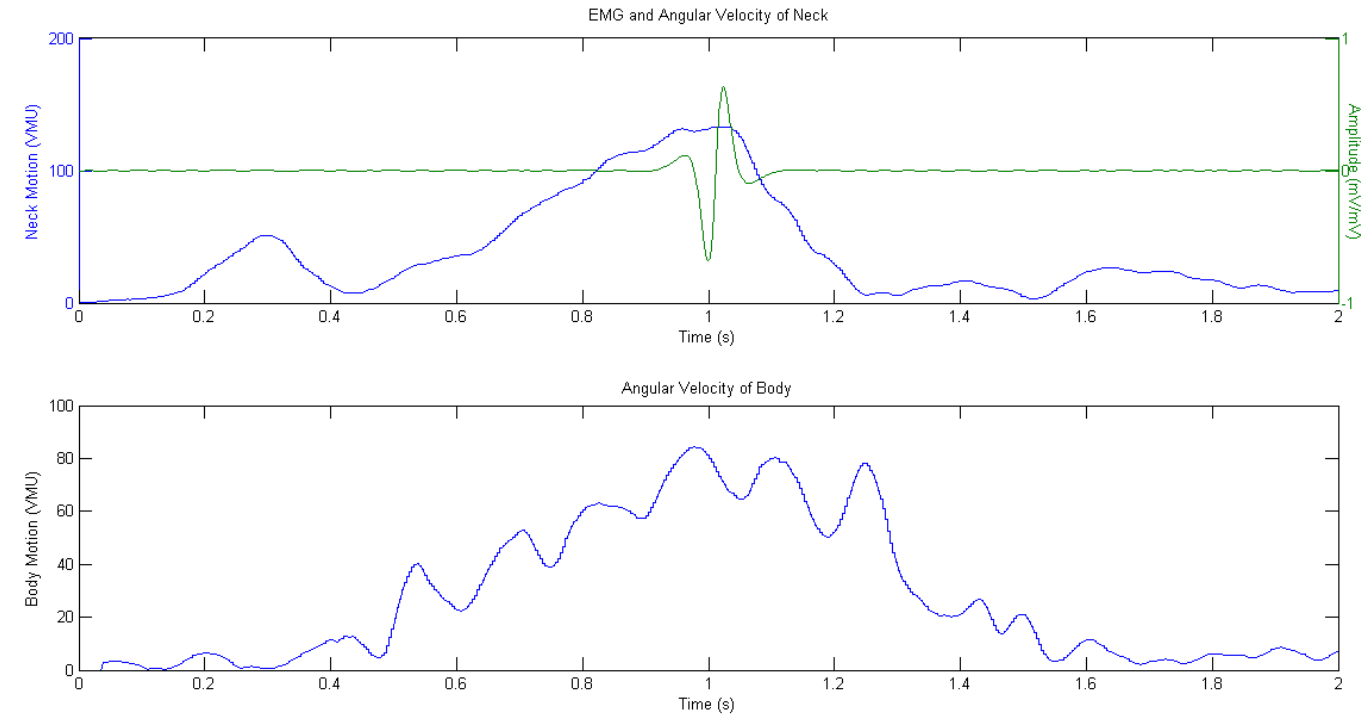

Figure 4.21: Comparison of EMG, Neck and Body Angular Velocity for Backward Rotation with Visuals (blue = motion; green $=\mathrm{EMG}$ ) 
Figure 4.22 shows all the differentials between neck and body motions. These shows a very similar pattern to the differentials computed for backward motion with no visuals. However, there were a couple of subjects that had slightly stronger signals resulting in slightly larger magnitudes from 0.6 to 1 second, as seen in the average in Figure 4.23.

\subsubsection{Forward Rotation}

This sections looks at the motion results during forward rotation. Figure 4.24, shows the motion profile of the neck, while VR was not present. The change in direction is visible by the positive angular velocity values. Similar to backward rotation, there is very minimal linear acceleration. In this case, most of the linear acceleration of the neck occurs after motion. The green line shows acceleration in the backward pitch plane as the neck makes up for the forward push during jerk. Linear acceleration across the yaw and pitch planes remain minimal. The angular velocity graph in Figure 4.24 shows an initial jerk in the opposite direction of motion.

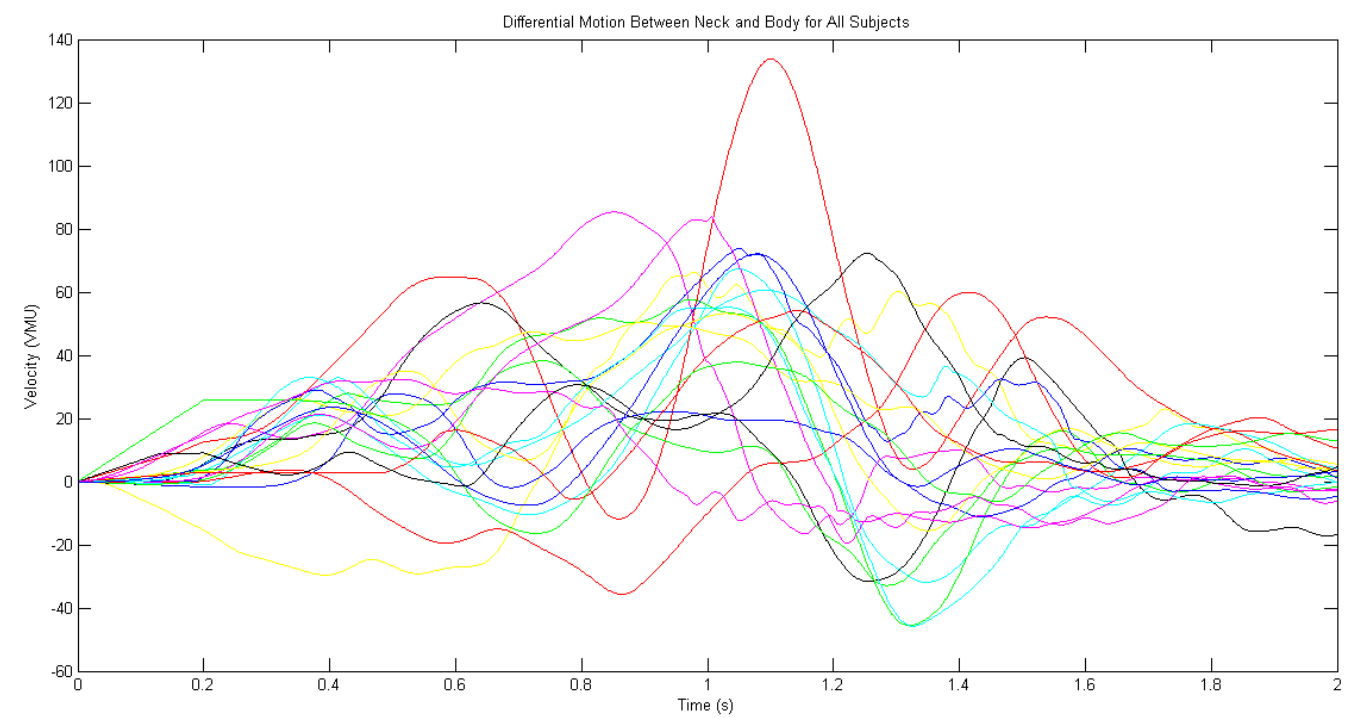

Figure 4.22: All Differential Motion Graphs for Backward Rotation with Visuals 

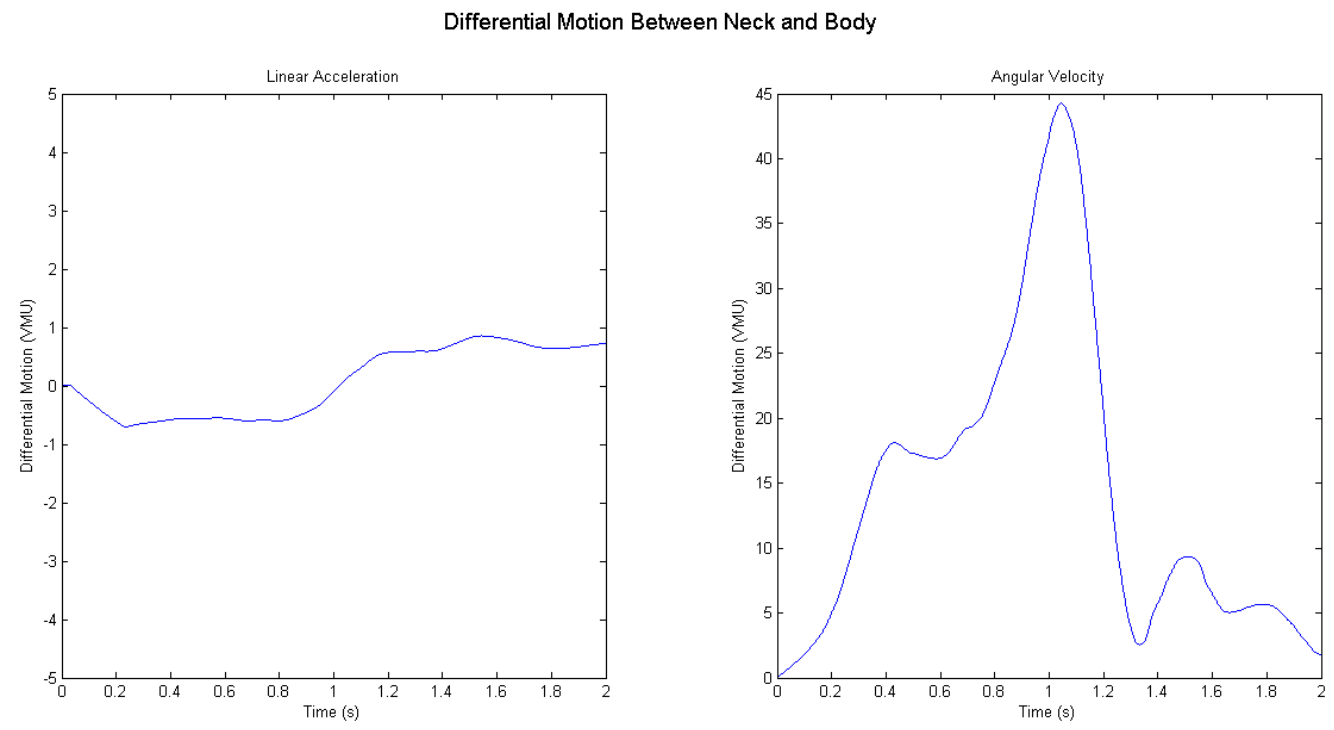

Figure 4.23: Average Differential Motion Graphs for Backward Rotation with Visuals
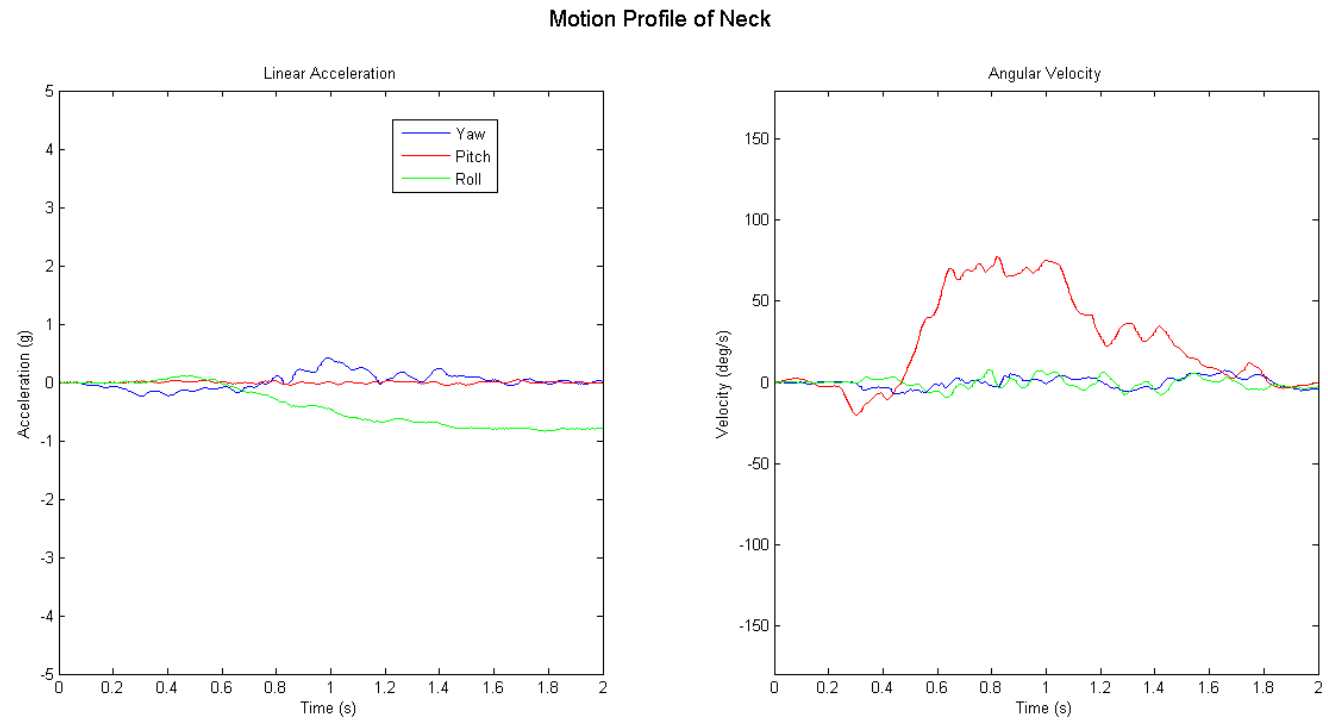

Figure 4.24: Motion Profile of Neck for Forward Rotation with no Visuals (red = pitch; blue = yaw; green $=$ roll $)$ 
However, the magnitude of this initial peak in smaller than the ones recorded during backward motion. This may be due to the support provided by the headrest as the head makes its initial movement backward. Another difference seen in the angular velocity is a greater period of close to constant angular velocity (0.6 - 1 second).

Figure 4.25 shows the motion profile of the body. The body shows majority of linear acceleration during motion, rather than after. The greatest motion occurs along the roll plane, as the body is pushed forward. In the angular velocity graph, there is no initial motion in the opposite motion, as seen in the neck profile. The body is directly pushed in the direction of motion. The angular velocity of the body not only reaches a maximum that exceeds the heights reached in the backward motion, it also exceeds the maximum angular velocity of the neck. The swaying of the body remains constant as the body changes its centre of mass to refrain from tipping.

Figure 4.26, shows the sum of angular motion for both the neck and body. It evident that the body reaches a higher angular motion than the neck. The body also shows more frequent oscillations, during motion and after. Figure 4.27 plots the differentials between neck and head motions for all subjects. This plot shows a slightly different pattern than the differential plots for the backward motion. The graph starts off with greater body motion during motion, followed by greater neck motion after motion. This is further illustrated by the average differential curve in Figure 4.28. The body definitely showed a greater degree of motion in the forward pitch plane, however the amount of neck motion is slightly lower compared to the differential magnitudes seen in the backward motion. This is caused by the upper body being flung backward during the initial jerk. For forward rotation, the differential peak angular velocity of the head decreases to about $60 \%$ of the differential peak angular velocity seen during backward motion. However, dominance in body angular velocity increases by $15 \%$.

When looking at the results for forward movement with VR, the linear acceleration graph in Figure 4.29 shows forward acceleration. The angular velocity graphs shows a very similar overall pattern as seen in Figure 4.24. There is period of constant angular velocity from 0.6 to 1 seconds. A very slight difference can be seen in the minor oscillations during constant velocity. 
Motion Profile of Body
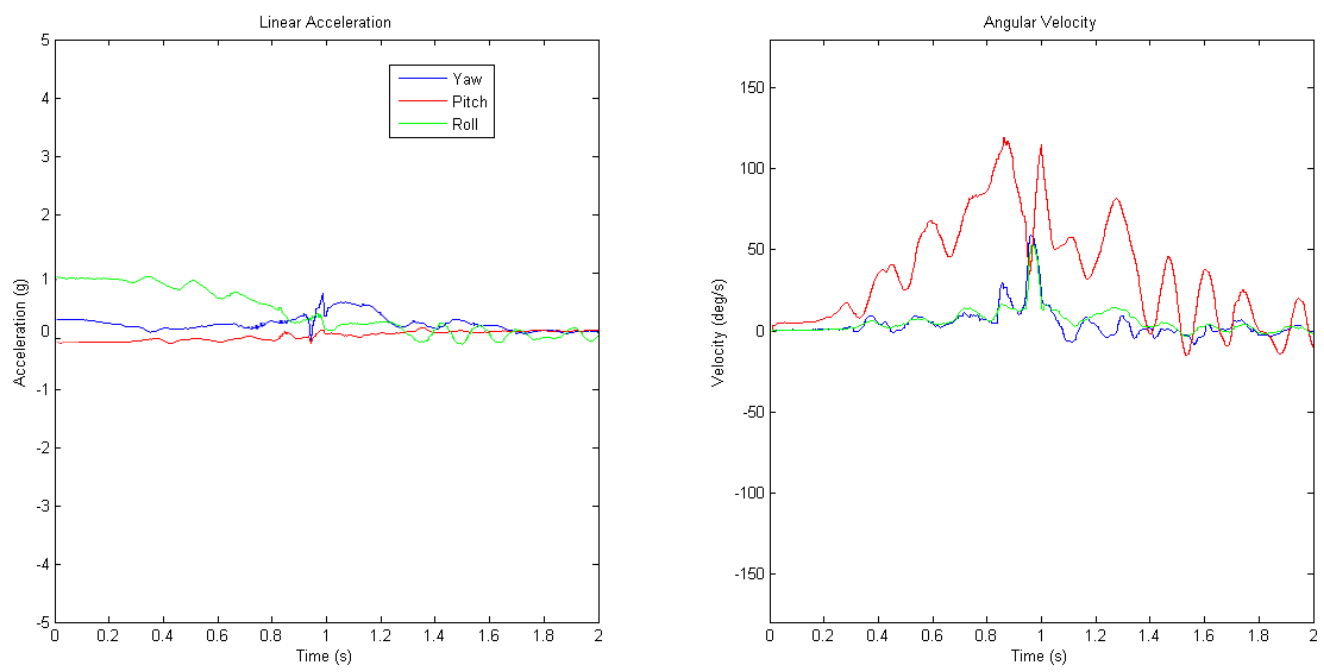

Figure 4.25: Motion Profile of Body for Forward Rotation with no Visuals (red = pitch; blue = yaw; green $=$ roll)
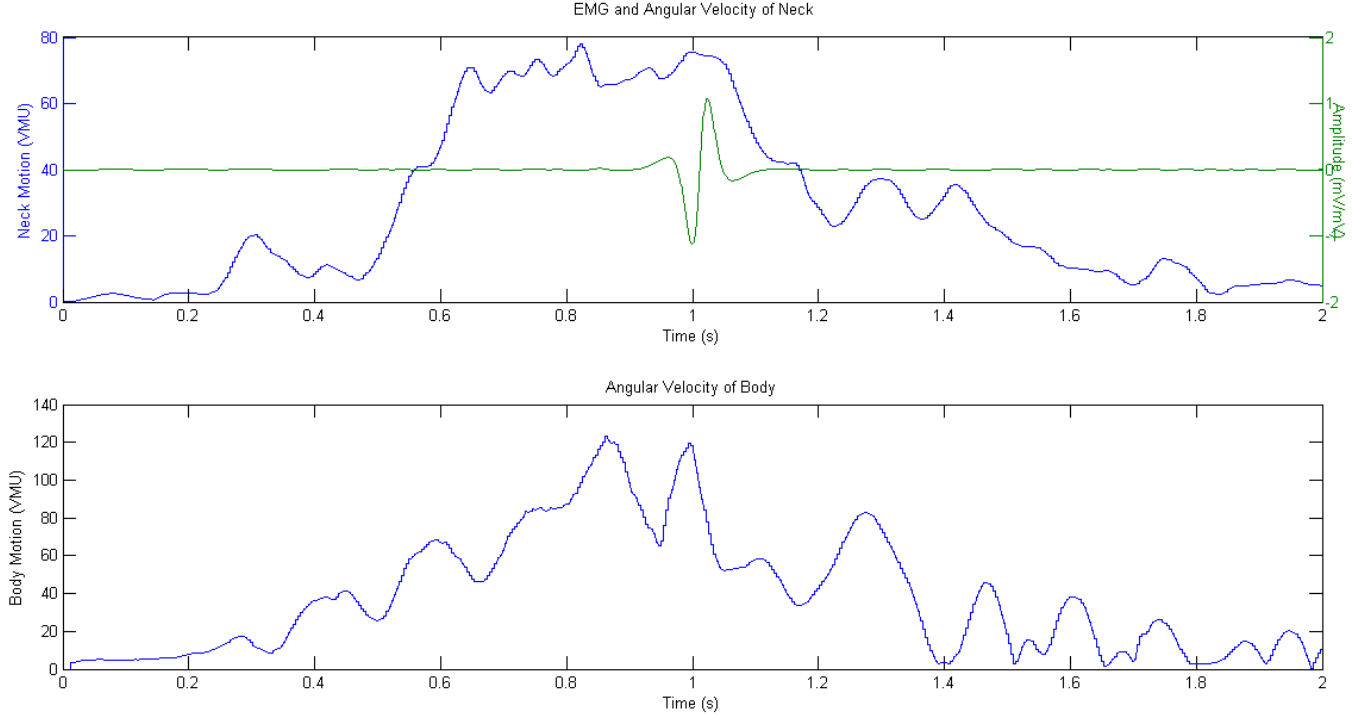

Figure 4.26: Comparison of EMG, Neck and Body Angular Velocity for Forward Rotation with no Visuals $($ blue $=$ motion; green $=\mathrm{EMG}$ ) 


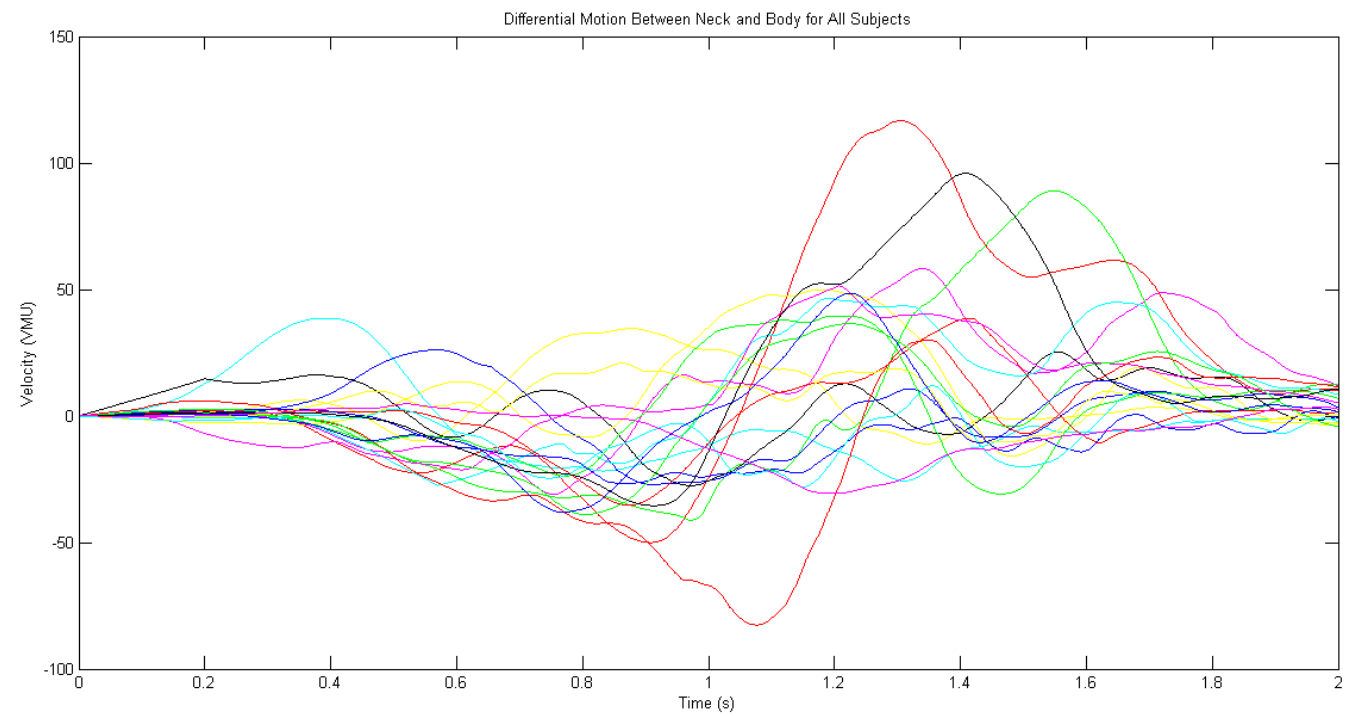

Figure 4.27: All Differential Motion Graphs for Forward Rotation with no Visuals

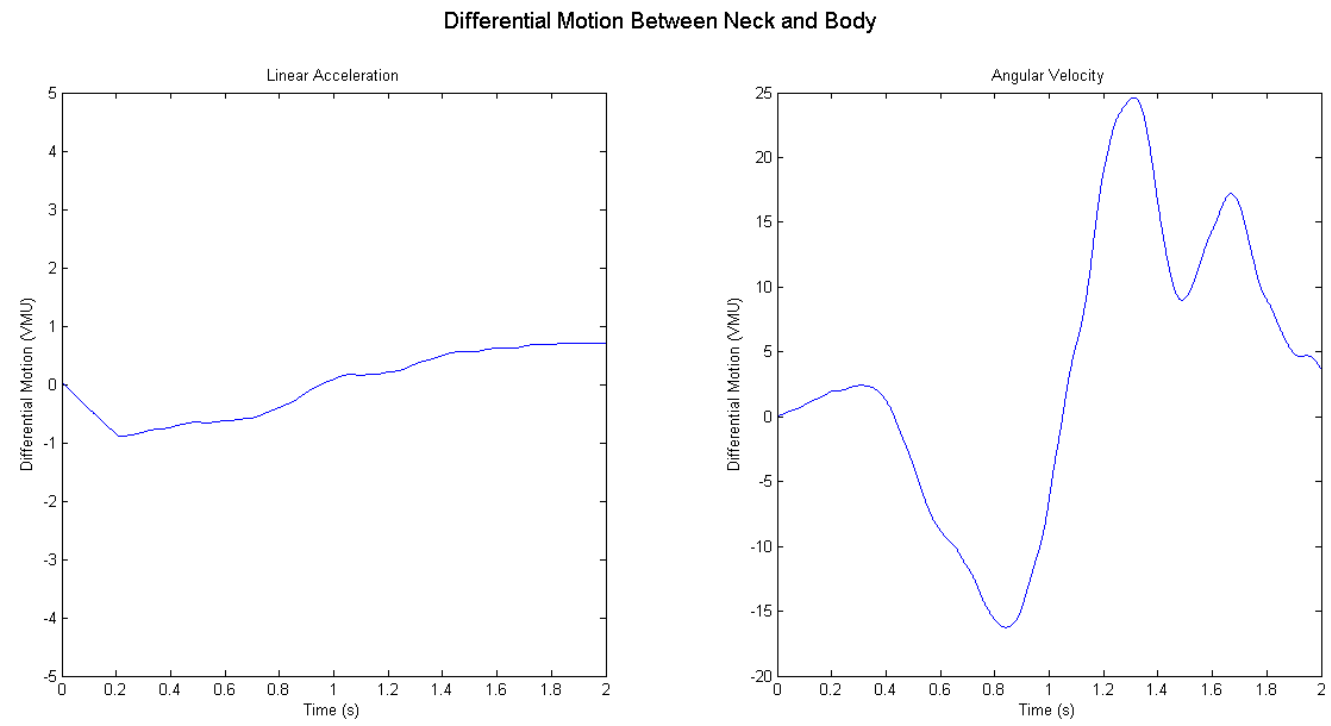

Figure 4.28: Average Differential Motion Graphs for Forward Rotation with no Visuals 
These oscillations are slightly more prominent and constant, compared to the random ones seen in Figure 4.24.

The motion profile of the body, seen in Figure 4.30, does not show any noticeable differences when VR was present. The maximum angular velocity reaches close to 100 degrees per second and the amount of bodying swaying is similar to the graphs above. The comparison graph in Figure 4.31, shows that the body motion reaches a higher magnitude compared to neck motion. When comparing this graph to the ones seen during backward motion, the overall pattern for the body motion is similar. However, the neck motion shows a longer period of constant angular velocity during forward motion.

Figure 4.32 plots the differentials between neck and body for all subjects during forward rotation, with the presence of VR. When looking at the average between all subjects in Figure 4.33, the body has dominance during motion, followed by neck dominance after the final jerk. During backward rotation, the neck had greater dominance throughout. The maximum amplitudes are relatively similar with and without the presence of VR. However, movement during backward rotation showed greater maximum magnitudes of motion, compared to forward motion.

\subsubsection{EMG Data}

Table 4.2 summarizes the average quantitative results obtained from all the subjects' EMG. An extensive table showing results for each specific muscle can be found in Appendix A. In order to be able to compare data between subjects with different muscle strengths, statistical values were taken as a percentage of the MVC. These values further explain the patterns noticed in the graphs above. When comparing the values between the four different conditions, the forward rotation showed higher results. The presence of visuals resulted in slightly higher values compared to no visuals.

Higher iEMG values for the forward rotation and the presence of VR demonstrates a higher amount of overall work performed by the neck muscles. Furthermore, the higher RMS values for these conditions signifies a higher muscle power exerted by the neck muscles. The average effect muscle power exhibited during forward motion was approximately $10-10.5 \%$ of the MVC. 
Motion Profile of Neck
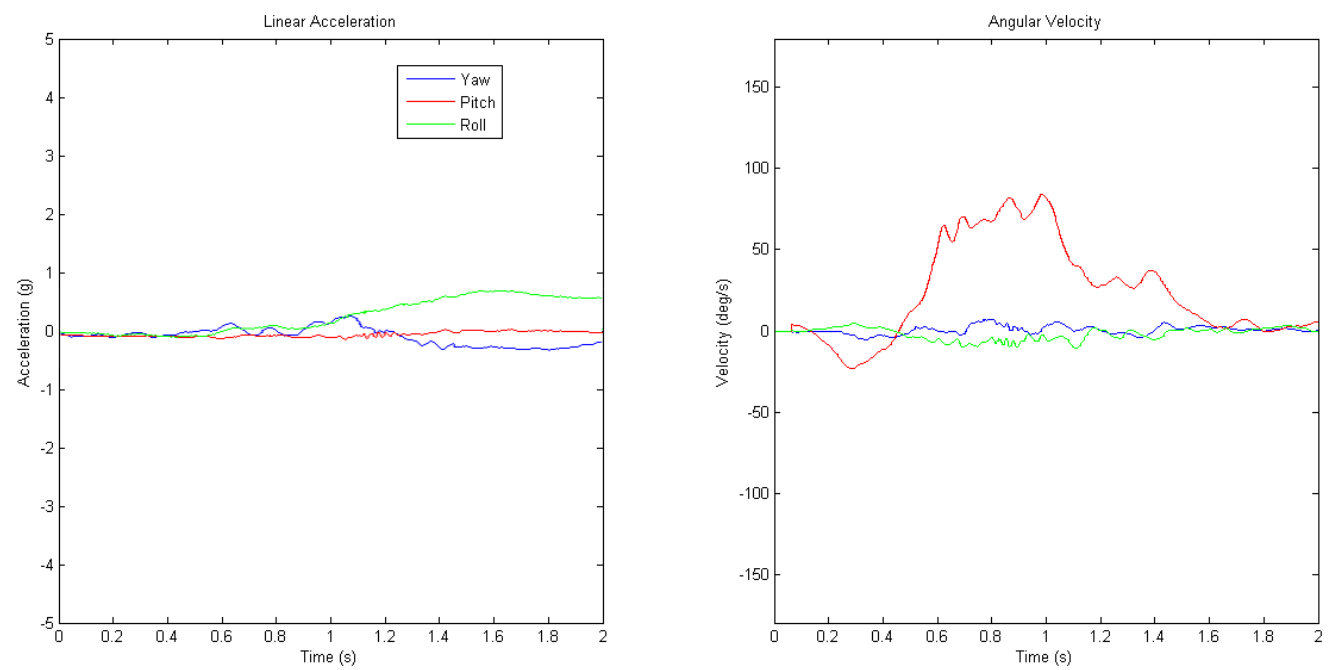

Figure 4.29: Motion Profile of Neck for Forward Rotation with Visuals (red = pitch; blue = yaw; green $=$ roll $)$

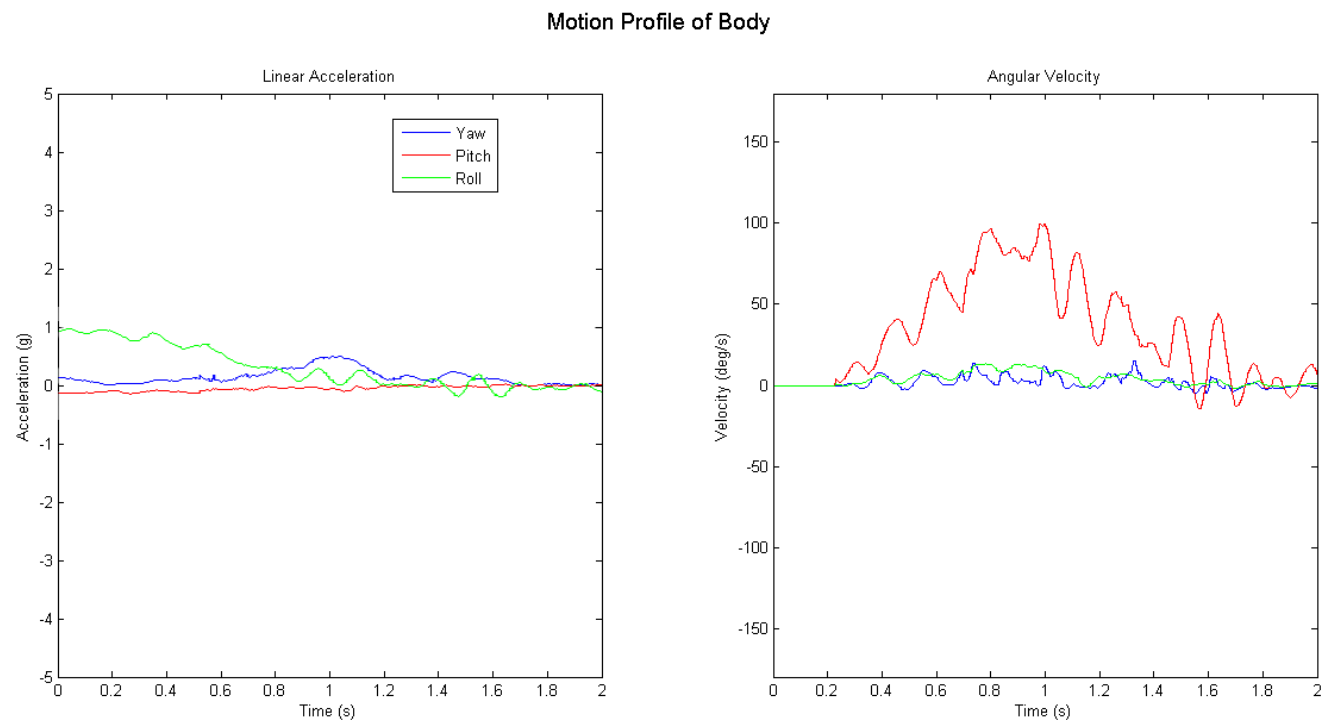

Figure 4.30: Motion Profile of Body for Forward Rotation with Visuals (red = pitch; blue = yaw; green $=$ roll) 

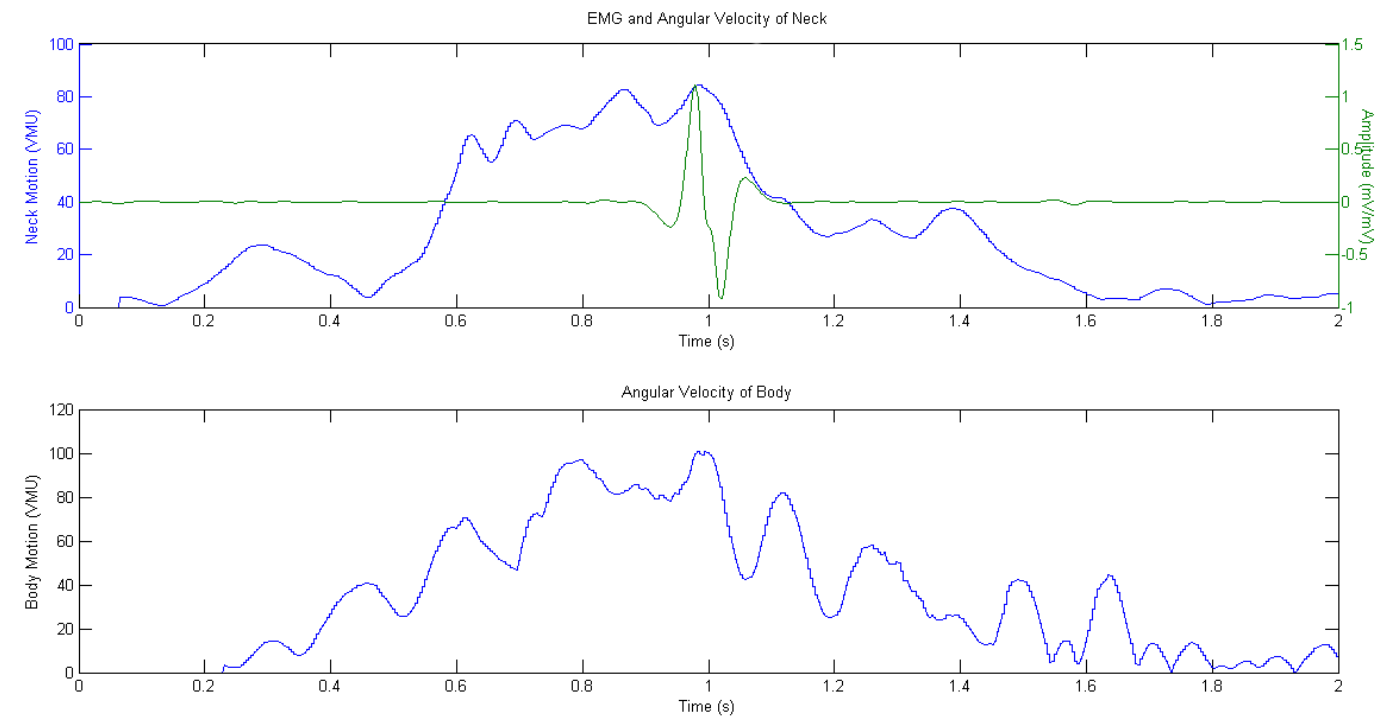

Figure 4.31: Comparison of EMG, Neck and Body Angular Velocity for Forward Rotation with Visuals $($ blue $=$ motion; green $=\mathrm{EMG})$

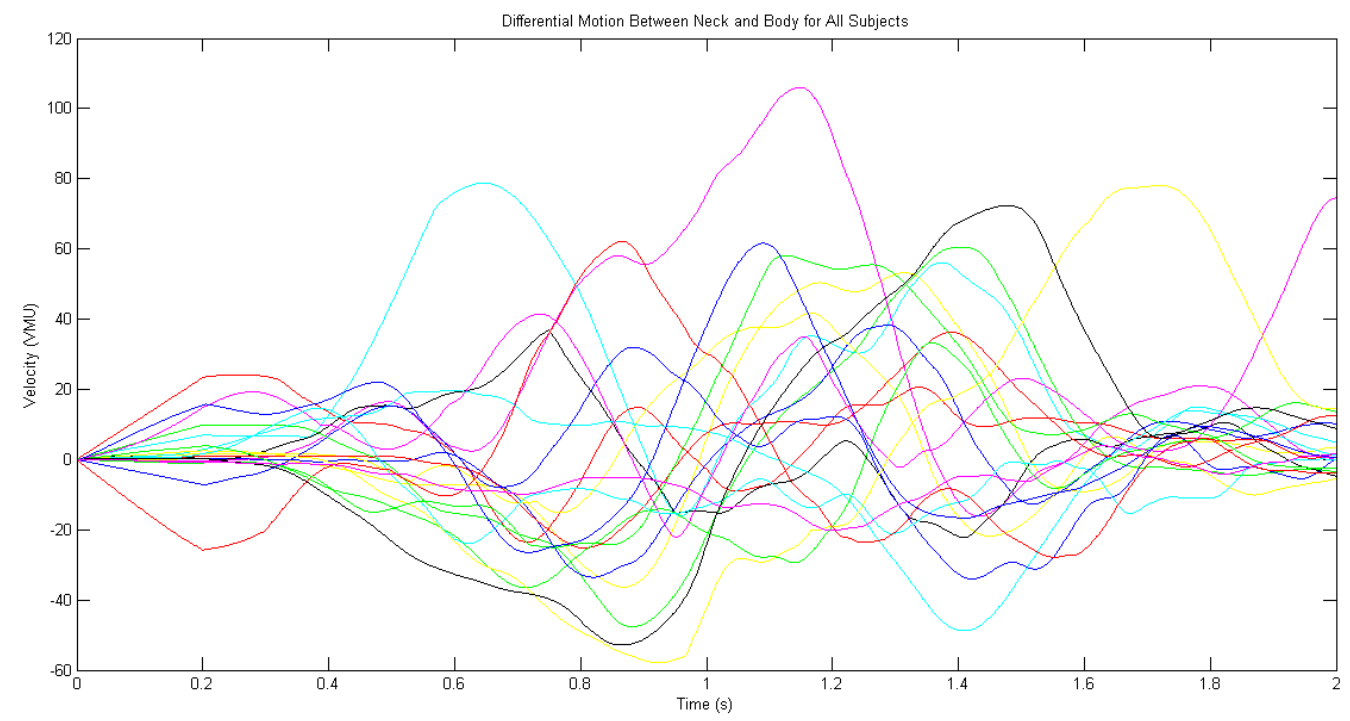

Figure 4.32: All Differential Motion Graphs for Forward Rotation with Visuals 

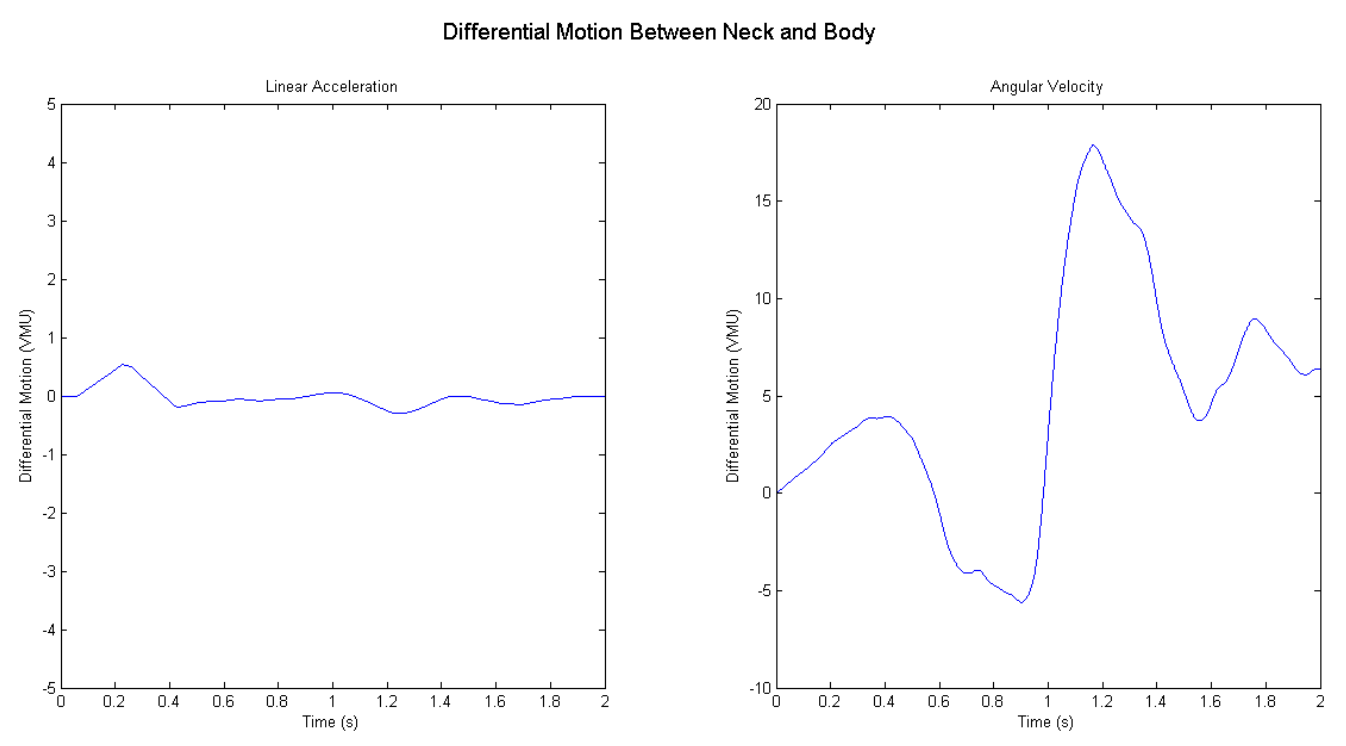

Figure 4.33: Average Differential Motion Graphs for Forward Rotation with Visuals

The average effect muscle power exerted by the neck muscles was about 7.5-8\% of the MVC. Since the variances and standard deviations provide information related to how disperse the EMG values were from the mean, higher variances and standard deviations indicates an increase in phasic activity.

Tables A.2 and A.4 in Appendix A show that during the backward motion, the RMS, variance, and iEMG values were greatest for the TRAP muscles, followed by the SCM muscles having the second highest, and the SCAL muscles having the lowest. However, for the forward rotation, the RMS, variance, and iEMG values slightly decrease for the TRAP muscles, while they increase for the SCM and SCAL muscles. This indicates that the amount of work and strength of contractions do vary depending on the muscle. The posterior neck muscles are more active during the backward motion and the anterior neck muscles are most active during forward motion. The ZC, SCC, and WL values further highlights the patterns of the EMG. The increase in activity during forward rotation can be identified by a greater number of times the EMG passes the zero mark, as well as the number of times the signal changes slopes signs. 


\subsection{Tabulated Results}

Table 4.1 indicates the units for each feature.

Table 4.1: Units Chart

\begin{tabular}{|c|c|}
\hline EMG Feature & Units \\
\hline RMS & $(\%$ of MVC $)$ \\
\hline Absolute Mean & $(\%$ of MVC $)$ \\
\hline Variance & $(\%$ of MVC $)$ \\
\hline Standard Deviation (SD) & $(\%$ of MVC $)$ \\
\hline Amplitude & $(\mathrm{mV} / \mathrm{mV})$ \\
\hline WL & $(\mathrm{mV} / \mathrm{mV})$ \\
\hline iEMG & $(\mathrm{mV} / \mathrm{mV}) \cdot \mathrm{s}$ \\
\hline Linear Acceleration & $(\mathrm{g})$ \\
\hline Angular Velocity & $($ degrees $/ \mathrm{second})$ \\
\hline Differential Linear Acceleration & VMU \\
\hline Differential Angular Velocity & VMU \\
\hline
\end{tabular}

Another interesting point to notice was that for conditions with no visuals, the right muscles showed slightly more activity than the left muscles. It would be interesting to test if this is related to hand dominance. Moreover, when visuals were present the left muscles showed to be more active, with higher RMS and iEMG values. This can be explained by a slight rotation to the right as the subjects direct their focus toward the centre of the screen. The higher activity level of the TRAP and SCM muscles can also be seen by the higher number of ZC, SCC, and WL values.

\subsubsection{Motion Data}

Table 4.3 provides a summary of the motion data acquired from the head and body. Additional tables in appendix A provide more extensive results for each axes of motion. The 
Table 4.2: EMG Feature Average for Each Condition

\begin{tabular}{|l|c|c|c|c|}
\hline & $\begin{array}{c}\text { Backward no } \\
\text { VR }\end{array}$ & $\begin{array}{c}\text { Backward } \\
\text { with VR }\end{array}$ & $\begin{array}{c}\text { Forward no } \\
\text { VR }\end{array}$ & $\begin{array}{c}\text { Forward with } \\
\text { VR }\end{array}$ \\
\hline RMS & 7.64 & 8.00 & 10.17 & 10.55 \\
\hline Absolute Mean & 1.92 & 2.00 & 2.51 & 2.79 \\
\hline Variance & 0.63 & 0.81 & 1.12 & 1.18 \\
\hline SD & 7.80 & 8.12 & 10.32 & 10.60 \\
\hline Amplitude & 0.76 & 0.76 & 0.91 & 0.94 \\
\hline ZC & 61.17 & 73.83 & 67.17 & 75.30 \\
\hline SSC & 87.67 & 96.17 & 91.00 & 99.80 \\
\hline WL & 3.61 & 3.83 & 4.72 & 6.07 \\
\hline iEMG & 38.37 & 39.96 & 50.13 & 55.72 \\
\hline
\end{tabular}

linear acceleration amplitudes were very similar for conditions with and without the presence of VR. However, there was a slightly higher range of linear motion achieved during forward rotation. The head and body had very similar amount of linear acceleration. For angular velocity, the head reached higher angles of motion during backward motion, while the body reached higher angles during the forward motion. Although the muscle activity showed noticeable differences when visuals were present, the difference in motion between having visuals and no visuals were very minimal for both directions. This shows that the visuals mostly affect how the muscles react in order to achieve balance and stability.

Table 4.4 compares the subjects' height weight to the maximum differential motion between head and body. The results show that subjects with a lower head mass showed higher levels of motion, compared to subjects with a greater head mass. Thus, the head weight is also related to the degree of motion exerted during jerky rotational movements. 
Table 4.3: Motion Feature Average for Each Condition

\begin{tabular}{|c|c|c|c|c|}
\hline & $\begin{array}{c}\text { Backward no } \\
\text { VR } \\
\end{array}$ & $\begin{array}{c}\text { Backward with } \\
\text { VR }\end{array}$ & $\begin{array}{c}\text { Forward no } \\
\text { VR }\end{array}$ & $\begin{array}{c}\text { Forward with } \\
\text { VR } \\
\end{array}$ \\
\hline $\begin{array}{l}\text { Maximum } \\
\text { Linear Head } \\
\text { Acceleration } \\
\end{array}$ & 1.13 & 1.2 & 1.34 & 1.3 \\
\hline $\begin{array}{l}\text { Minimum } \\
\text { Linear Head } \\
\text { Acceleration }\end{array}$ & -0.18 & -0.11 & -0.37 & -0.2 \\
\hline $\begin{array}{l}\text { Maximum } \\
\text { Angular Head } \\
\text { Velocity }\end{array}$ & 37.84 & 50.6 & 75.81 & 79.35 \\
\hline $\begin{array}{l}\text { Minimum } \\
\text { Angular Head } \\
\text { Velocity }\end{array}$ & -94.97 & -129.52 & -22.16 & -28.26 \\
\hline $\begin{array}{l}\text { Maximum } \\
\text { Linear Body } \\
\text { Acceleration }\end{array}$ & 1.08 & 1.15 & 1.4 & 1.24 \\
\hline $\begin{array}{l}\text { Minimum } \\
\text { Linear Body } \\
\text { Acceleration } \\
\end{array}$ & -0.19 & -0.2 & -0.77 & -0.75 \\
\hline $\begin{array}{l}\text { Maximum } \\
\text { Angular Body } \\
\text { Velocity }\end{array}$ & 19.53 & 9.16 & 113.83 & 96.98 \\
\hline $\begin{array}{l}\text { Minimum } \\
\text { Angular Body } \\
\text { Velocity }\end{array}$ & -77.58 & -82.7 & -20.75 & -17.21 \\
\hline $\begin{array}{l}\text { Maximum } \\
\text { Differential } \\
\text { Acceleration } \\
\end{array}$ & 0.58 & 0.97 & 0.7 & 0.55 \\
\hline $\begin{array}{l}\text { Minimum } \\
\text { Differential } \\
\text { Acceleration }\end{array}$ & -0.31 & -0.8 & -0.96 & -0.34 \\
\hline $\begin{array}{l}\text { Maximum } \\
\text { Differential } \\
\text { Velocity } \\
\end{array}$ & 38.52 & 44.78 & 24.68 & 18.07 \\
\hline $\begin{array}{l}\text { Minimum } \\
\text { Differential } \\
\text { Velocity }\end{array}$ & -2.31 & 0 & -16.25 & -5.92 \\
\hline
\end{tabular}


Table 4.4: Head Weight to Motion Comparison

\begin{tabular}{|r|r|}
\hline $\begin{array}{r}\text { Head } \\
\text { Weight }\end{array}$ & $\begin{array}{r}\text { Maximum } \\
\text { Motion } \\
\text { (VMU) }\end{array}$ \\
\hline 14.48 & 36.58 \\
\hline 13.20 & 36.65 \\
\hline 10.40 & 61.42 \\
\hline 11.20 & 85.33 \\
\hline 13.50 & 35.97 \\
\hline 15.60 & 24.36 \\
\hline 10.56 & 50.45 \\
\hline 8.80 & 41.77 \\
\hline 14.80 & 27.29 \\
\hline 13.60 & 23.62 \\
\hline 11.60 & 50.98 \\
\hline 10.80 & 51.09 \\
\hline 12.48 & 34.35 \\
\hline 11.12 & 40.84 \\
\hline 11.20 & 43.26 \\
\hline 14.40 & 27.47 \\
\hline 12.00 & 36.12 \\
\hline 12.80 & 38.98 \\
\hline 12.00 & 31.25 \\
\hline 11.12 & 42.27 \\
\hline
\end{tabular}

\subsection{Discussion}

The results show that there are observable differences between how neck muscles react, and how the head and body work to stabilize posture when rotated in the forward and backward pitch plane. In experiments performed by Forslund et al. trunk rotations averaged about 45 degrees for the transfer of SCI patients from table to wheelchair [14]. Other studies found that transferring patients from wheelchair to shower chairs resulted in lateral trunk rotations of about 30 degrees [16, 64]. Hence, the rotations used in this study is the maximum about of movement patients should experience, as they undergo their day to day activities. 
Neck rotations seen in this study match the sustained angles found to cause neck discomfort in [65]. The neck EMG obtained in this study had magnitudes that were very similar to other studies relating to neck analysis during whiplash [12, 17, 45, 66]. However, by analyzing rotational motion in the forward and backward pitch plane, results showed differences in the strength of contractions produced by the different muscles analyzed. As seen in [47], the posterior neck muscles were most active when moved in the posterior direction and the anterior neck muscles were most active when moved in the anterior direction. However, there were also differences found between anterior neck muscles themselves. The larger SCM muscles provided stronger contractions compared to the smaller SCAL muscles. Furthermore, the highest energy regions were found to be at the very end of motion. This confirms that the greatest force was exhibited at the stop of motion, as the neck worked to maintain control and posture. The EMG showed much lower values during motion. Additionally, trials with VR present showed a difference in neck posture, as the subjects were focused towards the screen.

Furthermore, the difference in motion profiles amongst the subjects may also be affected by the body and head weights of the subjects, so the subjects' weights were also recorded. When the head weight of the subjects were approximated at $8 \%$ of the body weight, according to [67], and compared to the head and body motion, the body motion did not seem to be affected by the body weight. This may be due to the harness that prevents the body from moving past a certain degree. However, one of the interesting things to note was that, subjects that had lower head weight showed noticeably higher degrees of head motion during backward rotation. In backward motion there is a headrest for support and the only major movement found to occur before the muscles start contracting is during the initial jerk. The difference in movement during the jerk caused by the sudden start of motion compared to the sudden stop, can be explained by the higher level of unawareness of upcoming motion in the beginning. Whereas, the stop of motion is expected after 1 second. Hence, this is an important factor to consider in further research. In addition, during the forward motion, the motion profile starts off with body dominance, followed by neck dominance. During backward rotations, the head showed greater dominance throughout the ride. 
Studies by Siegmund et al. showed similar neck muscle and motion behaviour during linear motion [47]. However, this project was able to pick up on additional patterns and factors that influenced neck muscle and motion behaviours during rotational movements. Previous studies have indicated that spatial orientation is greatly affected by combined sensory systems, as they add more stimulus inputs $[18,19]$. This project has showed that it also affects neck muscle responses. The EMG signal processing techniques successfully produced clean signals that highlighted muscle behaviour from the beginning to the end of motion. Additionally, the adaptive filter technique, commonly used for ECG processing, was also shown to effectively removed ECG crosstalk from the raw EMG signals.

Maximum effective muscle power and work of $10.54 \%$ and $55.72(\mathrm{mV} / \mathrm{mV}) \cdot \mathrm{s}$ were reached respectively. The minimum muscle power and work value, relative to MVC, were $7.63 \%$ and $38.37(\mathrm{mV} / \mathrm{mV}) \cdot \mathrm{s}$ respectively. Both the maximum values were from forward trials with VR present, while both the minimum value were from backward trials without the presence of VR. Thus, these results provide the standard amounts of muscle power and work exerted by healthy subjects, as they undergo rotational movements. These results can now be compared to patients with neck disorders, conditions, and/or weaker neck muscles.

\subsubsection{Comparison to Previous Experimentation}

Since the previous experiments conducted on abdomen and back muscles used longer durations of motion, this study focused on having a shorter duration of motion, so that the head and body movement and muscle behaviour could be analyzed specifically during sudden increases and decreases of speed. There were three main differences that were evident between this study and the previous study relating to abdomen and back muscles during rotational motions [48]. The first difference, as mentioned earlier, was the ECG crosstalk that occurred in neck EMG. The second difference was the slightly smaller magnitudes seen in the contractions. This is expected as the neck works to control a smaller weight portion of the body (the head), where as the abdomen and back muscles work to control a larger weight. The head is estimated at about $8.2 \%$ of the total body weight, whereas the abdomen is estimated at about $13 \%$ of the total body weight [67]. The final difference was the direction and magnitudes of head motion, 
relative to body motion. The similarities between the two studies were the increase in muscle activity during forward rotations and trials with VR presence.

\subsubsection{Limitations}

The application of this study focuses on rotational motions that disabled individuals using wheelchairs experience in their daily lives. One of the assumptions taken was that the motions experienced while travelling on a wheelchair were caused by another person pushing the wheelchair from behind. Hence, the subject may not always be aware of the upcoming motions. Performing the same tests where the subject has control of motion (as they do when they travel on motorized wheelchairs) may produce different results. Moreover, although this study tested the maximum amount of rotation a patient would typically experience in the pitch motion, at times they may also experience rotational motions in the roll and yaw planes, for example when turning corners.

Also, this study focuses on the behaviour and motion of healthy individuals only. Wheelchairs users are highly prone to much weaker muscles and responses. However, it is important to first get an idea of how healthy muscles react to such motions, in order to able to determine how weaker muscles differ from the healthy. Patients with neck injuries tend to hold rigid postures to prevent further pain during movement. Overtime, this starts affecting posture and adds to neck strain. Furthermore, here, a motion simulator was used to simulate wheelchair motion indoors. There may be other factors that influence how patients may react to such motions, such as the environment, weather, etc.

Additionally, when the tests were executed with VR, the subjects were seated slightly left to the center of the screen. This was because the motion simulator was a two person ride, which resulted in each seat being positioned slightly to the side of the center. The tunnel scenery played during trials with VR, presented the illusion of travelling forwarded through the tunnel. Since the visual scene of the tunnel showed common motion during forward and backward rotations, there was conflicting visual input for backward motion. Finally, the display presented to the subjects was a 2 dimensional tunnel view. This differs from the typical street view that wheelchair users experience realistically. However, as a preliminary study, the purpose was to find out whether visual stimuli had an effect on how neck muscles reacted. 


\subsection{Chapter 4 Summary}

This chapter has illustrated how neck muscle reactions differ under various conditions, such as direction of motion and the presence of VR. Taking the differential between head and body motion has helped provide useful information relating to motion dominance. Motions patterns were found to mimic common patterns seen in head and body motion during whiplash injuries. Tabulated results further supported the hypothesis mentioned in section 1.2. This chapter has described in detail the muscle contraction, head and body motion ranges that healthy individuals typically produce during rotational motions. 


\section{Chapter 5}

\section{Conclusions and Future Work}

\subsection{Conclusions}

$\mathrm{T}$

THIS study has successfully tested and analyzed neck muscle behaviour along with head and body movement during rotational motions, with and without the presence of VR. The controlled variables, direction and visuals have shown to play a role in neck muscle responses. The signal processing techniques used provided clean comparable signals and the adaptive filtering technique was able to effectively remove ECG crosstalk from EMG signals.

The use of a motion simulator and VR can be further used for rehabilitation, balance and posture training purposes. Through this study it has become clear how the head and body work together to reach stabilization during motion. Furthermore, factors such as visuals, head weight, and direction of motion have been found to affect how neck muscles react during motion. Hence, these factors must be taken into consideration not only when planning rehabilitation program, but also when designing wheelchairs, headrests, and other supportive neck braces.

The greatest neck muscle activity was found to be at the end of motion. The strength of contractions depended on the location of the muscle and the direction of motion. The posterior neck muscles produced stronger contractions when subjects were rotated in the backward pitch plane, and the anterior neck muscles produced stronger contractions when subjects were rotated in the forward pitch plane. Maximum effective muscle power and work of $10.54 \%$ and 55.7 $(\mathrm{mV} / \mathrm{mV}) \cdot \mathrm{s}$ were reached respectively, while the minimum muscle power and work value, relative to MVC, were $7.63 \%$ and $38.3(\mathrm{mV} / \mathrm{mV}) \cdot s$ respectively. Trials with VR present resulted in higher muscle activity and work to reach stabilization. When the head and body motion were 
analyzed, results mimicked the S-phase of common whiplash injuries. While the head and body worked together to achieve stabilization during motion, the times of dominance between the two varied depending on the direction of motion. The neck had greater motion during backward motion. These results acquired from healthy subjects can now be compared to data acquired from patients with neck disorders or weaker neck muscles.

\subsection{Future Work}

Although motion simulators have proven to be effective devices to use for rehabilitation and training purpose, future research looking to study muscle behaviour and seated balance of wheelchair users should consider using actual wheelchairs for testing. Future work can use the results obtained from this study to analyze how the results of patients suffering from weaker muscles and/or neck disorders differ. Additionally, the EMG data ranges acquired from healthy subjects can also be used to functionally simulate weaker muscles, as a therapeutic option. Furthermore, since it is evident that visuals affect movement and muscle behaviour, it becomes important to analyze muscle behaviour of individuals with low vision. An additional sensory input to test would be sound. This would definitely be an interesting factor to test when studying EMG responses of the visually impaired.

Moreover, this study focuses on rotation over the pitch plane, as most motion during daily activities occur over this plane. However, wheelchair users may also be prone to slight motions in the roll and yaw planes. Thus, another future approach would be to test neck muscle activity, posture and balance during rotational motions in the roll and yaw planes, to see whether the muscle contraction strengths and work differs. Also, for future work the visual scenery can be modified to resemble streets the subjects typically travel. Furthermore, this study does not give the subjects control over motion. Neck muscle behaviour and seated balance of patients in motorized wheelchairs can be better examined by providing the test subjects control over motion.

Some of the features computed in this study including ZC, SCC, and WL provided useful information relating to EMG patterns. These features can also be used for purposes such as muscle disorder diagnosis and classification. 


\section{Appendix A}

\section{Experimental Results}

\section{A.1 Backward Rotation With No Visuals}

\section{A.1.1 EMG Data}

Table A.1: EMG Feature Data for Backward Rotation with no Visuals

\begin{tabular}{|l|c|c|c|c|c|c|}
\hline & SCM-L & SCM-R & SCAL-L & SCAL-R & TRAP-L & TRAP-R \\
\hline RMS & 6.11 & 8.49 & 5.18 & 8.73 & 7.82 & 9.48 \\
\hline Absolute Mean & 1.36 & 2.50 & 1.20 & 2.45 & 1.67 & 2.33 \\
\hline Variance & 0.41 & 0.74 & 0.28 & 0.74 & 0.67 & 0.95 \\
\hline SD & 6.38 & 8.57 & 5.34 & 8.58 & 8.21 & 9.73 \\
\hline Amplitude & 0.67 & 0.80 & 0.52 & 0.76 & 0.87 & 0.94 \\
\hline ZC & 62.00 & 58.00 & 65.00 & 61.00 & 62.00 & 59.00 \\
\hline SSC & 86.00 & 102.00 & 94.00 & 85.00 & 75.00 & 84.00 \\
\hline WL & 2.79 & 4.72 & 2.35 & 3.85 & 3.49 & 4.44 \\
\hline iEMG & 27.22 & 50.00 & 24.04 & 48.93 & 33.48 & 46.55 \\
\hline iEMG Sum & \multicolumn{7}{|c|}{230.23} \\
\hline
\end{tabular}




\section{A.1.2 Motion Data}

Table A.2: Motion Feature Data for Backward Rotation with no Visuals

\begin{tabular}{|l|c|c|c|}
\hline & Yaw & Pitch & Roll \\
\hline Maximum Linear Head Acceleration & 0.17 & 0.10 & 0.82 \\
\hline Minimum Linear Head Acceleration & -0.53 & -0.17 & -0.34 \\
\hline Maximum Angular Head Velocity & 16.42 & 37.41 & 9.70 \\
\hline Minimum Angular Head Velocity & -12.45 & -95.40 & -16.05 \\
\hline Maximum Linear Body Acceleration & 0.17 & 0.07 & 0.60 \\
\hline Minimum Linear Body Acceleration & -0.97 & -0.10 & -0.01 \\
\hline Maximum Angular Body Velocity & 16.85 & 11.54 & 7.26 \\
\hline Minimum Angular Body Velocity & -13.06 & -77.52 & -12.70 \\
\hline Maximum Differential Acceleration & & 0.53 & \\
\hline Minimum Differential Acceleration & & -0.29 & \\
\hline Maximum Differential Velocity & & 35.31 & \\
\hline Minimum Differential Velocity & & -45.47 & \\
\hline
\end{tabular}




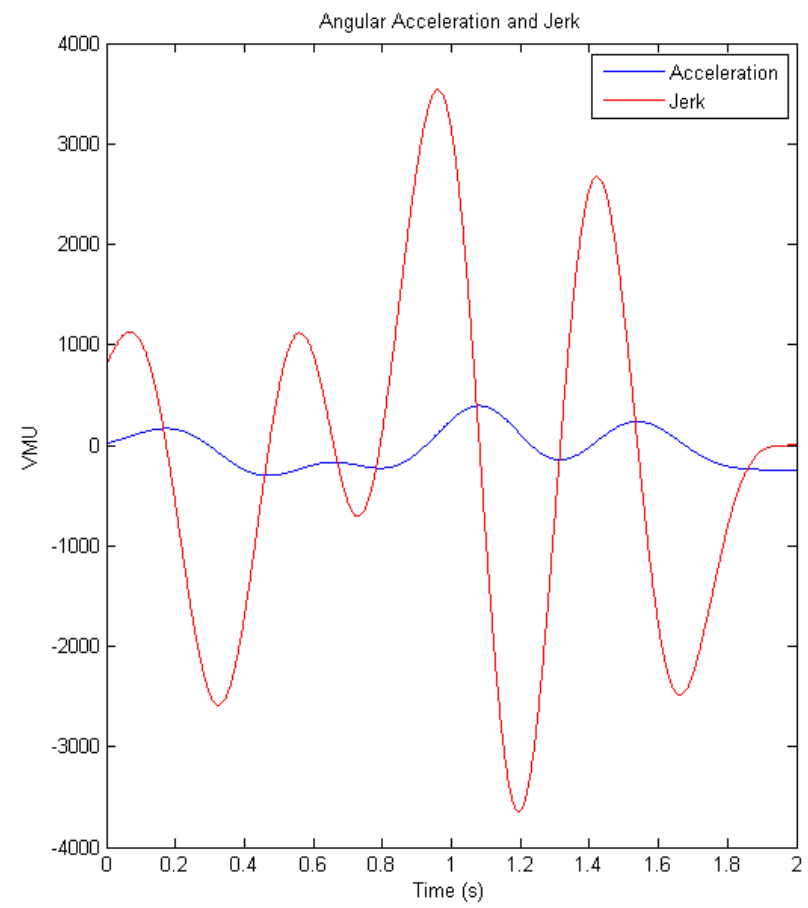

Figure A.1: Acceleration and Jerk Profiles of Neck for Backward Rotation with no Visuals

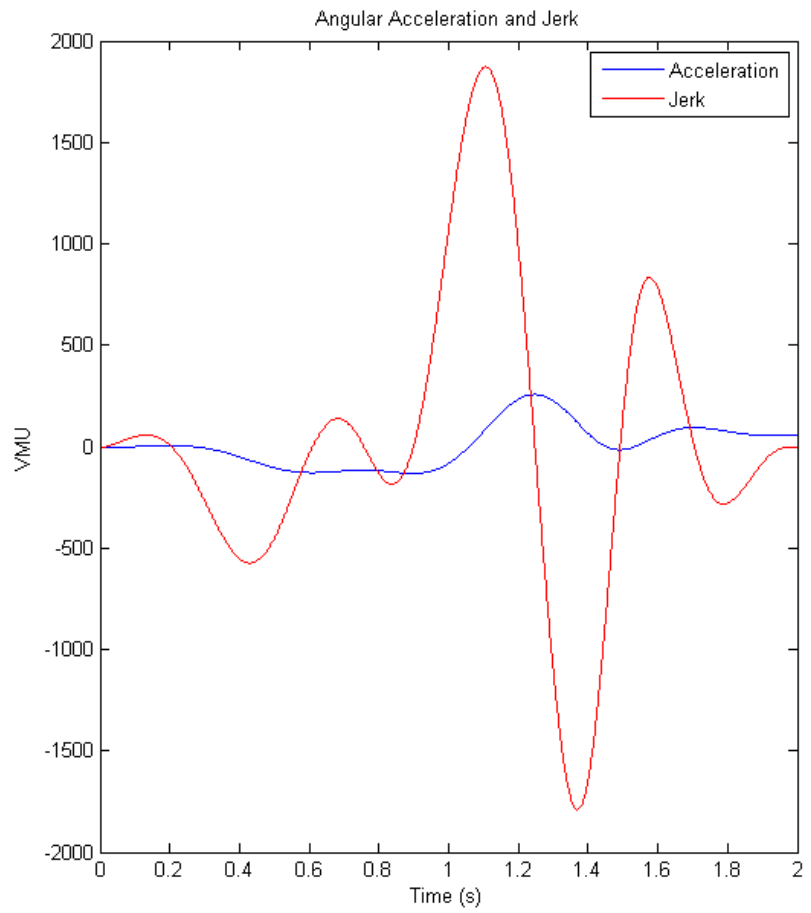

Figure A.2: Acceleration and Jerk Profiles of Body for Backward Rotation with no Visuals 


\section{A.2 Backward Rotation With Visuals}

\section{A.2.1 EMG Data}

Table A.3: EMG Feature Data for Backward Rotation with Visuals

\begin{tabular}{|l|c|c|c|c|c|c|}
\hline & SCM-L & SCM-R & SCAL-L & SCAL-R & TRAP-L & TRAP-R \\
\hline RMS & 7.50 & 7.99 & 3.76 & 3.94 & 14.59 & 10.20 \\
\hline Mean & 1.92 & 1.79 & 0.86 & 1.06 & 3.94 & 2.41 \\
\hline Variance & 0.59 & 0.66 & 0.15 & 0.15 & 2.21 & 1.10 \\
\hline SD & 7.66 & 8.12 & 3.87 & 3.88 & 14.87 & 10.48 \\
\hline Amp & 0.72 & 0.68 & 0.38 & 0.34 & 1.43 & 1.02 \\
\hline ZC & 69.00 & 86.00 & 86.00 & 70.00 & 59.00 & 73.00 \\
\hline SSC & 101.00 & 104.00 & 103.00 & 92.00 & 83.00 & 94.00 \\
\hline WL & 3.80 & 3.31 & 1.72 & 1.75 & 7.59 & 4.80 \\
\hline iEMG Max & 38.51 & 35.86 & 17.17 & 21.18 & 78.82 & 48.18 \\
\hline iEMG Sum & & & \multicolumn{2}{|c|}{239.73} & \\
\hline
\end{tabular}




\section{A.2.2 Motion Data}

Table A.4: Motion Feature Data for Backward Rotation with Visuals

\begin{tabular}{|l|c|c|c|}
\hline & Yaw & Pitch & Roll \\
\hline Maximum Linear Head Acceleration & 0.27 & 0.20 & 0.74 \\
\hline Minimum Linear Head Acceleration & -0.75 & -0.07 & -0.37 \\
\hline Maximum Angular Head Velocity & 8.24 & 50.29 & 17.27 \\
\hline Minimum Angular Head Velocity & -26.37 & -129.83 & -9.58 \\
\hline Maximum Linear Body Acceleration & 0.83 & 0.11 & 0.05 \\
\hline Minimum Linear Body Acceleration & -0.34 & -0.04 & -0.62 \\
\hline Maximum Angular Body Velocity & 5.92 & 8.36 & 3.91 \\
\hline Minimum Angular Body Velocity & -11.17 & -83.50 & -20.39 \\
\hline Maximum Differential Acceleration & & 0.90 & \\
\hline Minimum Differential Acceleration & & -0.78 & \\
\hline Maximum Differential Velocity & & 54.79 & \\
\hline Minimum Differential Velocity & & -52.92 & \\
\hline
\end{tabular}




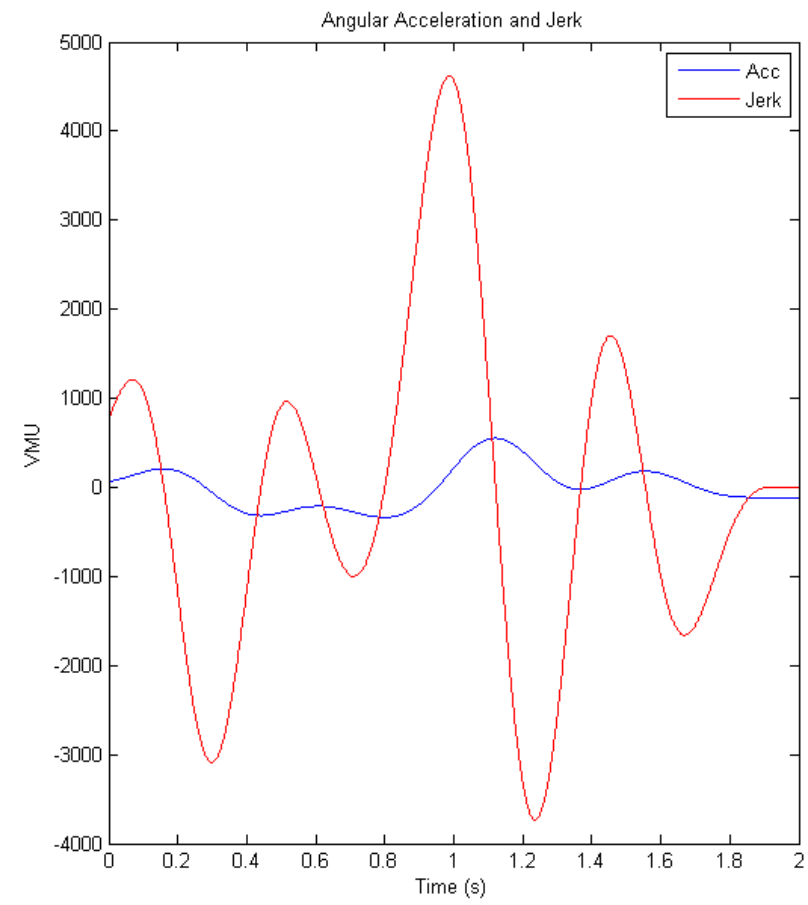

Figure A.3: Acceleration and Jerk Profiles of Neck for Backward Rotation with Visuals

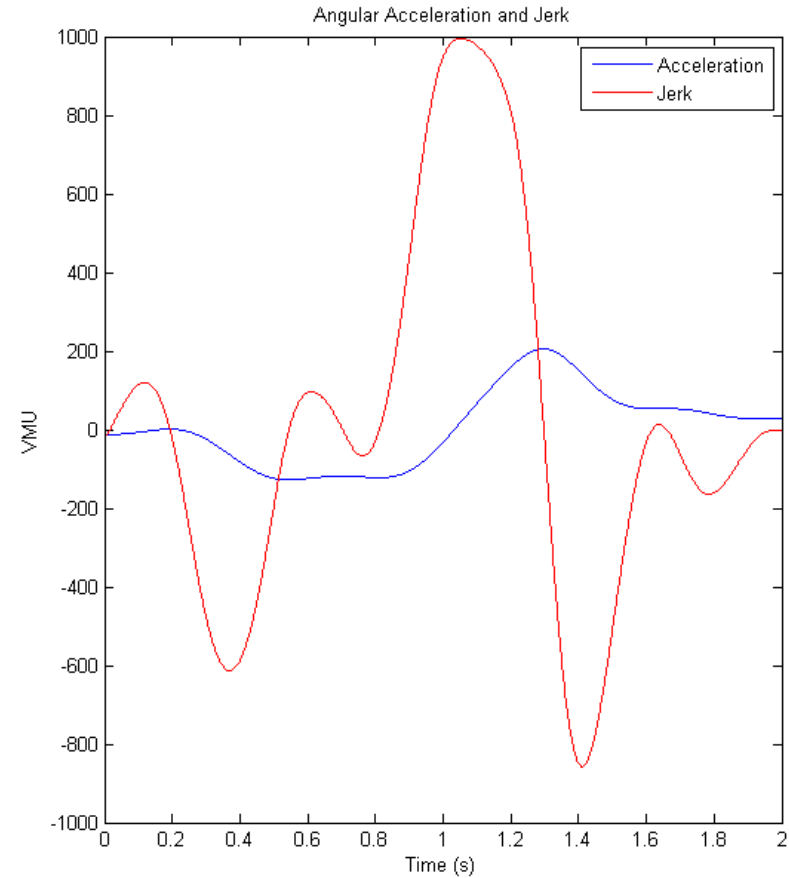

Figure A.4: Acceleration and Jerk Profiles of Body for Backward Rotation with Visuals 82 


\section{A.3 Forward Rotation With No Visuals}

\section{A.3.1 EMG Data}

Table A.5: EMG Feature Data for Forward Rotation with no Visuals

\begin{tabular}{|l|c|c|c|c|c|c|}
\hline & SCM-L & SCM-R & SCAL-L & SCAL-R & TRAP-L & TRAP-R \\
\hline RMS & 10.29 & 13.45 & 6.30 & 8.90 & 10.11 & 11.97 \\
\hline Mean & 2.58 & 3.08 & 1.48 & 2.55 & 2.44 & 2.91 \\
\hline Variance & 1.09 & 1.86 & 0.42 & 0.78 & 1.08 & 1.46 \\
\hline SD & 10.45 & 13.64 & 6.48 & 8.85 & 10.39 & 12.10 \\
\hline Amp & 0.96 & 1.12 & 0.63 & 0.75 & 1.01 & 0.99 \\
\hline ZC & 52.00 & 56.00 & 67.00 & 72.00 & 75.00 & 81.00 \\
\hline SSC & 85.00 & 77.00 & 99.00 & 93.00 & 87.00 & 105.00 \\
\hline WL & 4.85 & 5.49 & 2.96 & 4.83 & 4.86 & 5.35 \\
\hline iEMG Max & 51.64 & 61.61 & 29.53 & 51.10 & 48.76 & 58.13 \\
\hline iEMG Sum & \multicolumn{7}{|c|}{300.77} & & \\
\hline
\end{tabular}




\section{A.3.2 Motion Data}

Table A.6: Motion Feature Data for Forward Rotation with no Visuals

\begin{tabular}{|l|c|c|c|}
\hline & Yaw & Pitch & Roll \\
\hline Maximum Linear Head Acceleration & 0.43 & 0.05 & 0.12 \\
\hline Minimum Linear Head Acceleration & -0.25 & -0.05 & -0.84 \\
\hline Maximum Angular Head Velocity & 7.39 & 77.82 & 7.93 \\
\hline Minimum Angular Head Velocity & -7.26 & -20.14 & -9.46 \\
\hline Maximum Linear Body Acceleration & 0.65 & 0.05 & 0.93 \\
\hline Minimum Linear Body Acceleration & -0.19 & -0.22 & -0.23 \\
\hline Maximum Angular Body Velocity & 59.39 & 119.45 & 53.47 \\
\hline Minimum Angular Body Velocity & -8.36 & -15.14 & -3.17 \\
\hline Maximum Differential Acceleration & & 0.73 & \\
\hline Minimum Differential Acceleration & & -0.93 & \\
\hline Maximum Differential Velocity & & 14.88 & \\
\hline Minimum Differential Velocity & & -41.07 & \\
\hline
\end{tabular}




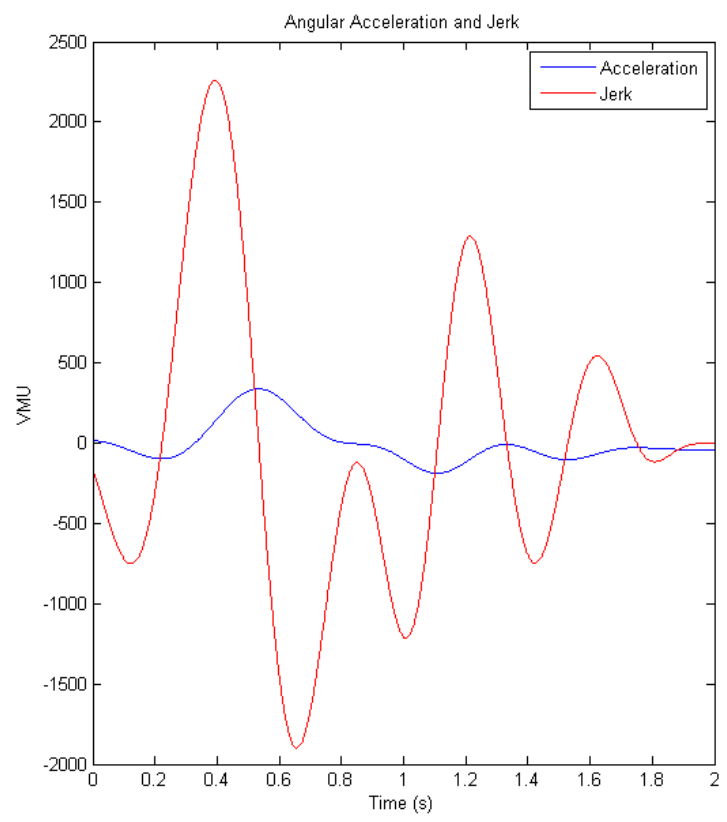

Figure A.5: Acceleration and Jerk Profiles of Neck for Forward Rotation with no Visuals

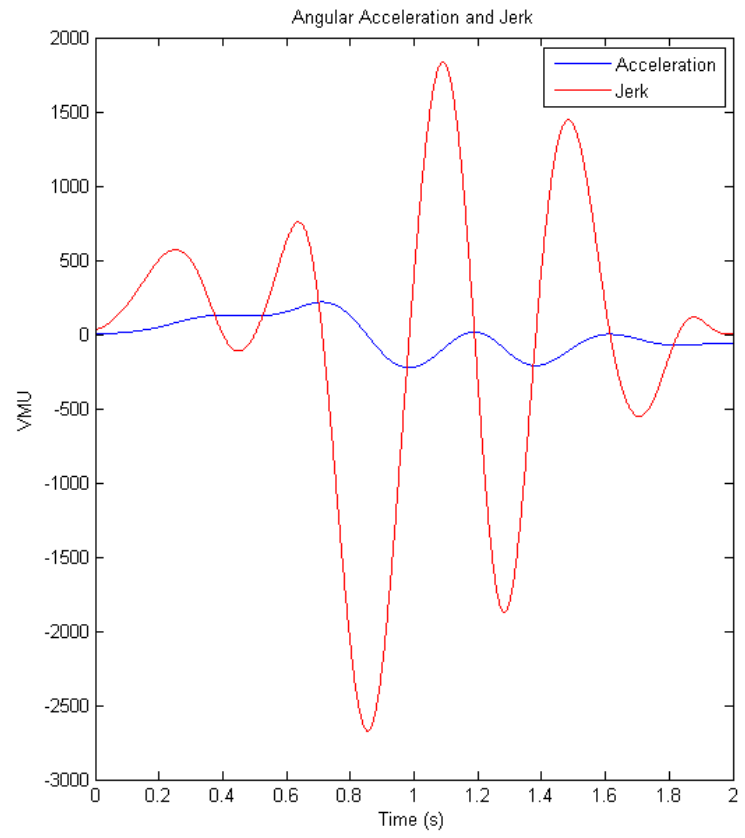

Figure A.6: Acceleration and Jerk Profiles of Body for Forward Rotation with no Visuals 


\section{A.4 Forward Rotation With Visuals}

\section{A.4.1 EMG Data}

Table A.7: EMG Feature Data for Forward Rotation with Visuals

\begin{tabular}{|l|c|c|c|c|c|c|}
\hline & SCM-L & SCM-R & SCAL-L & SCAL-R & TRAP-L & TRAP-R \\
\hline RMS & 13.90 & 13.38 & 7.64 & 8.43 & 9.39 & 10.55 \\
\hline Mean & 3.60 & 3.46 & 1.96 & 2.13 & 2.63 & 2.93 \\
\hline Variance & 1.99 & 1.73 & 0.61 & 0.71 & 0.90 & 1.12 \\
\hline SD & 14.12 & 13.15 & 7.81 & 8.45 & 9.48 & 10.59 \\
\hline Amp & 1.30 & 1.11 & 0.76 & 0.73 & 0.85 & 0.89 \\
\hline ZC & 68.00 & 70.00 & 75.00 & 86.00 & 70.00 & 83.00 \\
\hline SSC & 85.00 & 88.00 & 94.00 & 115.00 & 97.00 & 120.00 \\
\hline WL & 7.70 & 6.63 & 4.85 & 4.67 & 5.93 & 6.65 \\
\hline iEMG Max & 72.10 & 69.28 & 39.15 & 42.63 & 52.59 & 58.57 \\
\hline iEMG Sum & \multicolumn{7}{|c|}{334.32} & & \\
\hline
\end{tabular}




\section{A.4.2 Motion Data}

Table A.8: Motion Feature Data for Forward Rotation with Visuals

\begin{tabular}{|l|c|c|c|}
\hline & Yaw & Pitch & Roll \\
\hline Maximum Linear Head Acceleration & 0.33 & 0.09 & 0.75 \\
\hline Minimum Linear Head Acceleration & -0.27 & -0.09 & -0.05 \\
\hline Maximum Angular Head Velocity & 7.08 & 84.35 & 4.76 \\
\hline Minimum Angular Head Velocity & -5.80 & -23.25 & -10.62 \\
\hline Maximum Linear Body Acceleration & 0.50 & 0.02 & 0.96 \\
\hline Minimum Linear Body Acceleration & -0.01 & -0.15 & -0.19 \\
\hline Maximum Angular Body Velocity & 15.32 & 99.91 & 13.24 \\
\hline Minimum Angular Body Velocity & -4.94 & -14.28 & -2.20 \\
\hline Maximum Differential Acceleration & & 0.66 & \\
\hline Minimum Differential Acceleration & & -0.36 & \\
\hline Maximum Differential Velocity & & 13.49 & \\
\hline
\end{tabular}




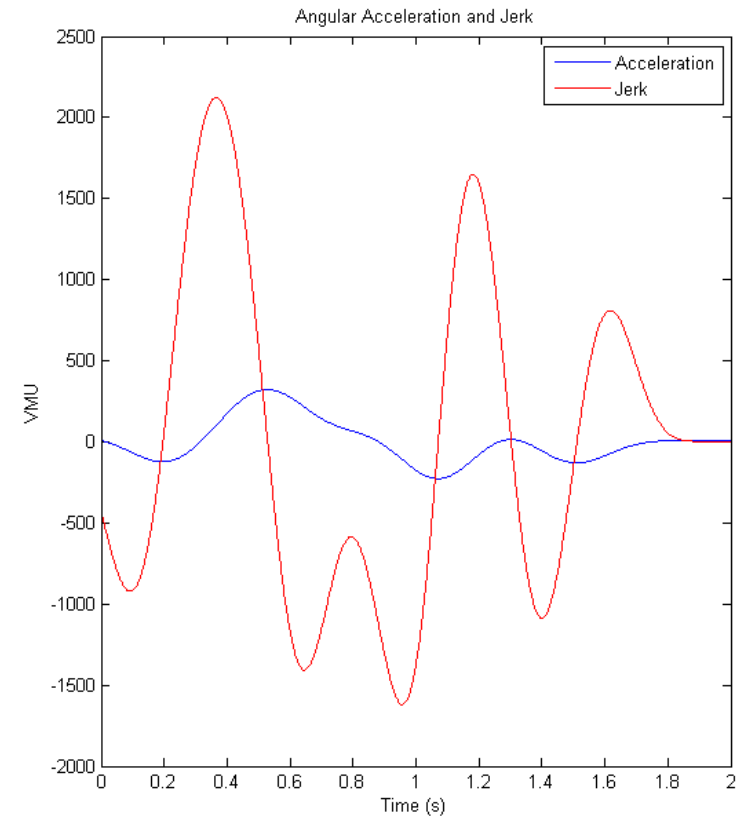

Figure A.7: Acceleration and Jerk Profiles of Neck for Forward Rotation with Visuals

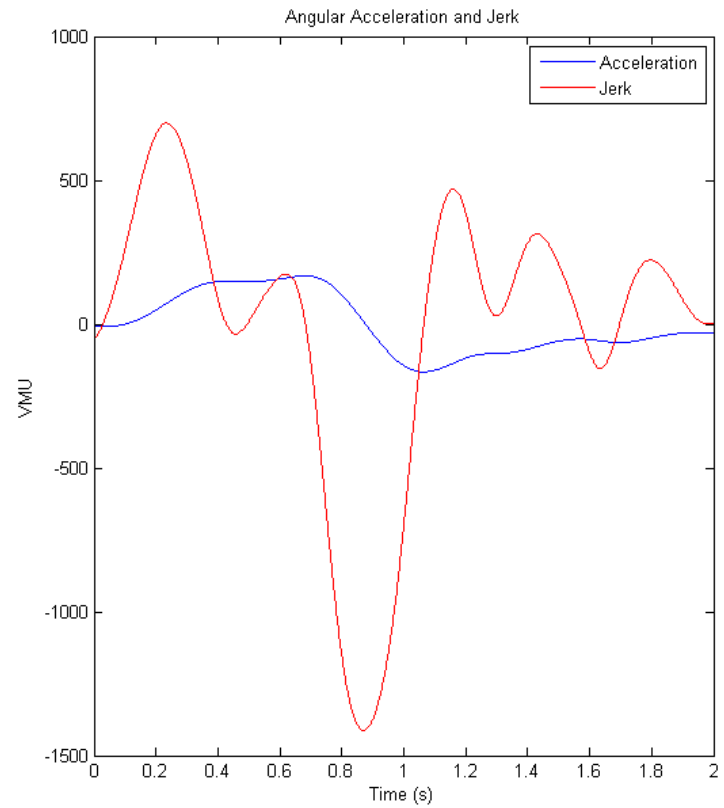

Figure A.8: Acceleration and Jerk Profiles of Body for Forward Rotation with Visuals 


\section{Appendix B}

\section{Publications}

\section{B.1 Conferences}

1. S. Sirikantharajah, K.M.V. McConville, Analysis of Neck EMG, Head Movement and Body Movement During Rotational Motion in a Motion Simulator, IEEE Engineering in Medicine and Biology Society 2015 conference (EMBC 2015), Aug. 2015, Milan, Italy (Under review).

2. N. Zolfaghari, M. Shafeie, S. Sirikantharajah, K.M.V. McConville, Comparison of PS25015A Dry Electrodes and Two Different $\mathrm{Ag} / \mathrm{AgCl}$ Wet Electrodes for ECG Applications, Sensor Devices Technologies and Applications (IARIA, 2014), Nov. 2014, pp.79-93, Lisbon, Portugal.

\section{B.2 Journals}

1. N. Zolfaghari, S. Sirikantharajah, M. Shafeie, K.M.V. McConville, Adaptive Filtering Technique and Comparison of PS25015A Dry Electrodes and Two Different Ag/AgCl Wet Electrodes for Wearable ECG Applications, Sensors and Transducers, 184(1), pp. 84-91, 2015. 


\section{Bibliography}

[1] Statistics Canada, "Injuries in Canada: Insights form the Canadian community health survey," Health at a Glance, Online, 2011. http://www.statcan.gc.ca/pub/82-624x/2011001/article/11506-eng.htm

[2] Sinnott, K.A., Milburn, P., McNaughton, H., "Factors associated with thoracic spinal cord injury, lesion level and rotator cuff disorders," Spinal Cord, vol. 38, pp. 748-753, 2000.

[3] Samuelsson K, Larsson H, Thyberg M, Gerdle B, "Wheelchair seating intervention," Disabil Rehabil, vol. 23, pp. 677-682, 2001.

[4] Samuelsson, K.A., Tropp, H., Nylander, E., Gerdle, B., "The effect of rear-wheel position on seating ergonomics and mobility efficiency in wheelchair users with spinal cord injuries: a pilot study," J. Rehabil. Res. Dev., vol. 41, pp. 65-74, 2004.

[5] Sanderson DJ, Sommer HJ, "Kinematic features of wheelchair propulsion," J Biomech, vol. 18, pp. 423-429, 1985.

[6] Rintala, D.H., Loubser, P.G., Castro, J., Hart, K.A., Fuhrer, M.J., "Chronic pain in a community-based sample of men with spinal cord injury: prevalence, severity, and relationship with impairment, disability, handicap, and subjective well-being," Arch. Phys. Med. Rehabil., vol. 79, pp. 604-614, 1996.

[7] Curtis, K.A., Drysdale, G.A., Lanza, R.D., Kolber, M., Vitolo, R.S., West, R., "Shoulder pain in wheelchair users with tetraplegia and paraplegia," Arch. Phys. Med. Rehabil., vol. 80, pp. 453-457, 1999. 
[8] Chow JW, Millikan TA, Carlton LG, Chae WS, Lim YT, Morse MI., "Kinematic and electromyographic analysis of wheelchair propulsion on ramps of different slopes for young men with paraplegia," Arch Phys Med Rehabil., vol. 90, pp. 271-278, 2009.

[9] Nederhand M.J., Hermens H.J., IJzerman M.J., Turk D.C., Zilvoid G., "Chronic neck pain disability due to an acute whiplash injury," U.S. National Library of Medicine, vol. 102, pp. 63-71, 2003.

[10] Allan G.B., "Anatomy and physiology of head and neck musculature," American Journal of Orthodontics, vol. 36, pp. 831-844, 1950.

[11] Jull G., Kristjansson E., Dall'Alba P., "Impairment in the cervical flexors: a comparison of whiplash and insidious onset neck pain patients," U.S. National Library of Medicine, vol. 9, pp. 89-94, 2004.

[12] Stapley P.J., Beretta M.V., Toffola E.D., Schieppati M., "Neck muscle fatigue and postural control in patients with whiplash injury," International federation of Clinical Neurophysiology, vol. 117, pp. 610-622, 2006.

[13] Middaugh S., Thomas K.J., Smith A.R., McFall T.L., Klingmueller J., "EMG biofeedback and exercise for treatment of cervical and shoulder pain in individuals with a spinal cord injury: A pilot study," Top Spinal Cord Inj. Rehabil., vol. 19, pp. 311-323, 2013.

[14] Forsland EB, Granström A, Levi R, Westgren N, Hirschfeld H, "Transfer from table to wheelchair in men and women with spinal cord injury: coordination of body movement and arm forces", Spinal Cord, vol. 45, pp. 41 - 84, 2007.

[15] Garg A, Owen B, Beller D, Banaag J, "A biomechanical and ergonomic evaluation of patient transferring task: bed to wheelchair and wheelchair to bed", Ergonomics, vol. 34, no. $3,289-312,1991$.

[16] Garg A, Owen B, Beller D, Banaag J, "A biomechanical and ergonomic evaluation of patient transferring task: wheelchair to shower chair and shower chair to wheelchair," Ergonomics, vol. 34, no. 4, 407 - 419, 1991. 
[17] Vibert N., MacDougall H.G., Waele C., Gilchrist D.P.D., Burgess A.M., Sidis A., Migliaccio A., Curthoys I.S., Vidal P.P., "Variability in the control of head movements in seated humans: A link with whiplash injuries," Journal of Physiology, vol. 532. pp. 851868. 2001.

[18] Mahajan HP, Dicianno BE, Cooper RA, Ding D, "Assessment of wheelchair driving performance in a virtual reality-based simulator," The Journal of Spinal Cord Medicine, vol. 36, no. 4, pp. $322-332,2013$.

[19] Cooper RA, Ding D, Simpson R, Fitzgerald SG, Spaeth DM, Guo S, Koontz AM, Cooper R, Kim J, Boninger ML, "Virtual reality and computer-enhanced training applied to wheeled mobility: an overview of work in Pittsburgh," Assistive Technology, vol. 17, no. 2, pp. $159-170,2005$.

[20] Inman DP, Loge K, Cram A, "Learning to drive a wheelchair in virtual reality," Journal of Special Education Technology, vol. 26, no. 3, pp. 21 - 34, 2011.

[21] Anonymous "Electromyogram," Encyclopedia of Neuroscience, vol. 2, pp. 1074, 2009.

[22] Anonymous "Chapter V: The Electromyogram," Acta Otolaryngol., vol. 49, pp. 51-53, 1958.

[23] R. Seeley, "Seeley's Anatomy \& Physiology," The McGraw-Hill Companies, 2011.

[24] Rangaraj M. Rangayyan, "Biomedical Signal Analysis a case-study approach”, 1st Edition, Johyn Wiley \& Sons - IEEE Press, Calgary, Canada, 2001.

[25] H. Davis, A. Forbes and L. Garceau, "THE ANALYSIS OF ELECTROMYOGRAM," Science (New York, N.Y.), vol. 75, pp. 436-437, 1932.

[26] Harvard Health Letter, "Whiplash," Academic OneFile, Online, Sept. 2009. http://go.galegroup.com/ps/i.do?id=GALE\%7CA207132860\&v=2.1\&u=rpu_main\&it=r\&p $=\mathrm{AONE} \& \mathrm{sw}=\mathrm{w} \& \mathrm{asid}=8 \mathrm{a} 552 \mathrm{c} 7481 \mathrm{~d} 5 \mathrm{ba506fb76909876660a6}$

[27] B. Brais, “Oculopharyngeal muscular dystrophy: A polyalanine myopathy," Curr. Neurol. Neurosci. Rep., vol. 9, no. 1, pp. 76-82, 2009. 
[28] L. Servais and G. Aubert, "Muscular Dystrophy," N. Engl. J. Med., vol. 371, no. 23, pp. e35, 2014.

[29] Steven H. Schot, "Jerk: The time rate of change of acceleration," American Journal of Physics, vol. 46, pp. 1090, 1978.

[30] W. Zhong, 钟 玮, R. Wu and 伍荣生, "Newtonian jerky dynamics and inertial instability," Acta Meteorologica Sinica, vol. 27, pp. 400-414, 2013.

[31] O. Umut, S. Yasar, "A Simple Jerky Dynamics, Genesio System," International Journal of Modern Nonlinear theory and Application, vol. 2, pp. 60-68, 2013.

[32] J. J. Lin, H. Abi-Rached, D. Kim, D. E. Parker and T. A. Furness, "A Natural Independent Visual Background Reduced Simulator Sickness," Human Factors and Ergonomics Society Annual Meeting Proceedings, vol. 46, pp. 2124, 2002.

[33] L. R. Harris, R. T. Dyde, M. R. Jenkin, "The use of visual and non-visual cues in updating the perceived position of the world during translation," Human Vision and Electronic Imaging, vol. 5666, 2005

[34] Weiquan Lu, B. -. H. Duh and S. Feiner, "Subtle cueing for visual search in augmented reality," in Mixed and Augmented Reality (ISMAR), 2012 IEEE International Symposium on, pp. 161-166, 2012.

[35] Y. Xiang, S. B. Yakushin and T. Raphan, "Modeling spatial tuning of adaptation of the angular vestibulo-ocular reflex," Experimental Brain Research, vol. 220, pp. 165-178, 2012.

[36] Zen-Chung Shih, Yuh-Sen Jaw and Mei-Ling Hsu, "Virtual roller coaster," in Virtual Reality, 2000. Proceedings. IEEE, pp. 225-232, 2000.

[37] EunHee Chang, InJae Hwang, Hyeonjin Jeon, Yeseul Chun, Hyun Taek Kim and Changhoon Park, "Effects of rest frames on cybersickness and oscillatory brain activity," in Brain-Computer Interface (BCI), 2013 International Winter Workshop on, pp. 62-64, 2013. 
[38] Sha Ma, M. Varley, L. Shark and J. Richards, "EMG biofeedback based VR system for hand rotation and grasping rehabilitation," in Information Visualisation (IV), 2010 14th International Conference, pp. 479-484, 2010.

[39] I. Cikajlo, A. Krpic and M. Gorisek-Humar, "Changes in EMG latencies during balance therapy using enhanced virtual reality with haptic floor," in Engineering in Medicine and Biology Society (EMBC), 2013 35th Annual International Conference of the IEEE, pp. 4129-4132, 2013.

[40] T. Ando, J. Okamoto and M. G. Fujie, "Development of roll-over support system with EMG control for cancer bone metastasis patients," in Robotics and Automation, 2007 IEEE International Conference on, pp. 1244-1249, 2007.

[41] S. Nakajima, S. Ino and T. Ifukube, "A preliminary study of MR sickness evaluation using visual motion aftereffect for advanced driver assistance systems," in Engineering in Medicine and Biology Society, 2007. EMBS 2007. 29th Annual International Conference of the IEEE, pp. 3044-3047, 2007.

[42] F. Öhberg, H. Grip, U. Wiklund, Y. Sterner, J. S. Karlsson, and B. Gerdle, "Chronic Whiplash Associated Disorders and Neck Movement Measurements: An Instantaneous Helical Axis Approach,” IEEE Trans. Inf. Technol. Biomed., vol. 7, no. 4, pp. 274-282, 2003.

[43] F. Ehtemam, P. a. Forbes, A. C. Schouten, F. C. T. Van Der Helm, and R. Happee, "Galvanic vestibular stimulation elicits consistent head-neck motion in seated subjects," IEEE Trans. Biomed. Eng., vol. 59, no. 7, pp. 1978-1984, 2012.

[44] L. M. Hynes and J. P. Dickey, "The rate of change of acceleration: Implications to head kinematics during rear-end impacts,” Accid. Anal. Prev., vol. 40, pp. 1063-1068, 2008.

[45] L. M. Hynes, N. S. Sacher, and J. P. Dickey, "Specific Parameters of the Perturbation Profile Differentially Influence the Vertical and Horizontal Head Accelerations During Human Whiplash Testing," Parallel Manip. New Dev., pp. 287-294, 2008. 
[46] C.-C. Yang, F.-C. Su, and L.-Y. Guo, "Comparison of neck movement smoothness between patients with mechanical neck disorder and healthy volunteers using the spectral entropy method," Eur. Spine J., vol. 23, pp. 1743-1748, 2014.

[47] G. P. Siegmund and J.-S. Blouin, "Head and neck control varies with perturbation acceleration but not jerk: implications for whiplash injuries.," J. Physiol., vol. 587, pp. 1829-1842, 2009.

[48] M. Shafeie, "Trunk muscle EMG in a specially designed virtual reality motion simulator, Master's thesis, University of Toronto, 2014.

[52] Utdallas, "Sensory systems V: Hearing \& balance."

[53] Szabo M, Kanuka H, "Effects of Violating Screen Design Principles of Balance, Unity and Focus on Recall learning, Study Time and Completion rate", Journal of Educational Multimedia and Hypermedia, vol. 8, no. 1, pp. 23 - 42, 1999.

[54] Rangayyan R.M., “The electrocardiogram (ECG),” Biomedical Signal Analysis. IEEE Press, pp. 14-28, 2002.

[55] Pizzuti G.P., Cifaldi S., Nolfe G., "Digital Sampling Rate and ECG Analysis," Journal of Biomedical Engineering., vol. 7, pp. 247-250, 1985.

[56] Rijnbeek P.R., Kors J.A., Witsenburg M., "Minimum Bandwidth Requirements for Recording of Pediatric Electrocardiograms," Circulation: Journal of the American Heart Association., vol. 104, pp. 3087-3090, 2001.

[57] Great Lakes NeuroTechnologies, "BioRadio: Owner's manual," 2014.

[58] Y. Wang et al., "Dry Electrode for the Measurement of Biopotential Signals," Science China, vol. 54, pp. 2435-2442, 2011.

[59] N. Zolfaghari, M. Shafeie, S. Sirikantharajah, K.M.V. McConville, Comparison of PS25015A Dry Electrodes and Two Different Ag/AgCl Wet Electrodes for ECG Applications, Sensor Devices Technologies and Applications, pp.79-93, 2014. 
[49] N. Zolfaghari, S. Sirikantharajah, M. Shafeie, K.M.V. McConville, Adaptive Filtering Technique and Comparison of PS25015A Dry Electrodes and Two Different $\mathrm{Ag} / \mathrm{AgCl}$ Wet Electrodes for Wearable ECG Applications, International Frequency Sensor Association, vol. 184, no. 1, pp. 84-91, 2015.

[50] F. R. Hashim, J. J. Soraghan, L. Petropoulakis, and N. G. N. Daud, "EMG Cancellation from ECG Signals using Modified NLMS Adaptive Filters,” pp. 8-10, 2014.

[51] Cypher Avenue, "Human Muscle Anatomy," Online, 2013. http://cypheravenue.com/wpcontent/uploads/2013/01/human-muscle-anatomy.jpg

[60] M. B. I. Reaz, M. S. Hussain and F. Mohd-Yasin, "Techniques of EMG signal analysis: detection, processing, classification and applications," Biol. Proc. Online, vol. 8, pp. 11-35, 2006.

[61] Yates R, Goodman J, "Probability and stochastic processes, a friendly introduction for electrical and computer engineers," John Wiley \& Sons, 2nd Edition, ISBN 0-471-27214-4, pp. $118-122,2005$.

[62] R. H. Chowdhury, M. B. I. Reaz, M. A. B. M. Ali, A. a a Bakar, K. Chellappan, and T. G. Chang, "Surface electromyography signal processing and classification techniques.," Sensors (Basel)., vol. 13, pp. 12431-12466, 2013.

[63] a. Phinyomark, a. Nuidod, P. Phukpattaranont, and C. Limsakul, "Feature extraction and reduction of wavelet transform coefficients for EMG pattern classification," Elektron. ir Elektrotechnika, vol. 122, no. 6, pp. 27-32, 2012.

[64] Zedka M, Kumar S, Narayan Y, "Electromyographic response of the trunk muscles to postural perturbation in sitting subjects", Journal of Electromyography and Kinesiology, vol. 8 , pp. 3 - 10, 1998.

[65] Kirby R. L., Fahie C. L., Smith C., Chester E. L., Macleod D. A., "Neck discomfort of wheelchair users: Effect of neck position," Disability and Rehabilitation, vol. 26, no. 1, pp. 9-15, 2004. 
[66] S. Vashisth and A. K. Salhan, "Real Time Acquisition of EMG Signal and Head Movement Recognition,” vol. 73, no. 1, pp. 19-22, 2013.

[67] Plagenhoef, S., Evans, F.G. and Abdelnour, T., "Anatomical data for analyzing human motion." Research Quarterly for Exercise and Sport, vol. 54, pp. 169-178, 1983. 


\section{Glossary}

$\begin{array}{ll}\text { BCR } & \text { BioCapture Recording File } \\ \text { COR } & \text { Centre of Rotation } \\ \text { CSV } & \text { Comma-Separated Values } \\ \text { DM1 } & \text { Myotonic Dystrophy } \\ \text { ECG } & \text { Electrocardiogram } \\ \text { EDMD } & \text { Emery-Dreifuss Muscular Dystrophy } \\ \text { EMG } & \text { Electromyogram } \\ \text { GVS } & \text { Galvanic Vestibular Stimulation } \\ \text { LGMD } & \text { Limb-Gridle Muscular Dystrophy } \\ \text { MAE } & \text { Motion After-Effect } \\ \text { MD } & \text { Muscular Dystrophy } \\ \text { MND } & \text { Mechanical Neck Disorders } \\ \text { MS } & \text { Multiple Sclerosis } \\ \text { MVC } & \text { Maximum Voluntary Contraction } \\ \text { OPMD } & \text { Oculopharyngeal Muscular Dystrophy } \\ \text { RSSQ } & \text { Revised Simulator Sickness Questionnaire } \\ \text { SCAL } & \text { Scalene } \\ \text { SCI } & \text { Spinal Cord Injury } \\ \text { SCM } & \text { Sternocleidomastoid } \\ \text { SD } & \text { Standard Deviation } \\ \text { SNR } & \text { Signal to Noise Ratio } \\ \text { SSC } & \text { Slope Sign Changes } \\ \text { TRAP } & \text { Trapezius } \\ \text { VMU } & \text { Vector Matrix Unit } \\ \text { VOR } & \text { Vestibular-Ocular Reflex } \\ \text { VR } & \text { Virtual Reality } \\ \text { WAD } & \text { Whiplash Related Disorders } \\ \text { WL } & \text { Waveform Lengths } \\ \text { ZC } & \text { Zero Crossings } \\ & \end{array}$

